\title{
Eating too much or too little : an FMRI food reward study of (ab)normal eating behaviour
}

Citation for published version (APA):

Siep, N. (2010). Eating too much or too little : an FMRI food reward study of (ab)normal eating behaviour. [Doctoral Thesis, Maastricht University]. Universitaire Pers Maastricht.

https://doi.org/10.26481/dis.20101104ns

Document status and date:

Published: 01/01/2010

DOI:

10.26481/dis.20101104ns

Document Version:

Publisher's PDF, also known as Version of record

\section{Please check the document version of this publication:}

- A submitted manuscript is the version of the article upon submission and before peer-review. There can be important differences between the submitted version and the official published version of record.

People interested in the research are advised to contact the author for the final version of the publication, or visit the DOI to the publisher's website.

- The final author version and the galley proof are versions of the publication after peer review.

- The final published version features the final layout of the paper including the volume, issue and page numbers.

Link to publication

\footnotetext{
General rights rights.

- You may freely distribute the URL identifying the publication in the public portal. please follow below link for the End User Agreement:

www.umlib.nl/taverne-license

Take down policy

If you believe that this document breaches copyright please contact us at:

repository@maastrichtuniversity.nl

providing details and we will investigate your claim.
}

Copyright and moral rights for the publications made accessible in the public portal are retained by the authors and/or other copyright owners and it is a condition of accessing publications that users recognise and abide by the legal requirements associated with these

- Users may download and print one copy of any publication from the public portal for the purpose of private study or research.

- You may not further distribute the material or use it for any profit-making activity or commercial gain

If the publication is distributed under the terms of Article $25 \mathrm{fa}$ of the Dutch Copyright Act, indicated by the "Taverne" license above, 


\section{EATING TOO MUCH OR TOO LITTLE}

AN FMRI FOOD REWARD STUDY OF (AB)NORMAL

EATING BEHAVIOUR 


\section{Colophon}

(c) 2010 Nicolette Siep, Maastricht

\section{Graphic design: Wim Siep}

Layout: Tiny Wouters

ISBN: 978-90-5278-984-2

Production: Datawyse, Universitaire Press Maastricht

All rights are reserved. No part of this book may be reproduced or transmitted in any form or by any means, without written permission from the author or, when appropriate, the publisher of the article. 


\title{
EATING TOO MUCH OR TOO LITTLE
}

\section{AN FMRI FOOD REWARD STUDY OF (AB)NORMAL EATING BEHAVIOUR}

\author{
Proefschrift \\ ter verkrijging van de graad van doctor aan de Universiteit Maastricht, \\ op gezag van de Rector Magnificus, Prof. mr. G.P.M.F. Mols, \\ volgens het besluit van het College van Decanen, \\ in het openbaar te verdedigen \\ op donderdag 4 november 2010 om 14:00 uur
}

door

Nicolette Siep 


\section{Promotor}

Prof. dr. A.T.M. Jansen

\section{Copromotores}
Dr. A. Roefs
Dr. A. Roebroeck

\section{Beoordelingscommissie}

Prof. dr. A. R. Arntz (voorzitter)

Prof. dr. R.A.H. Adan (UMC, Utrecht)

Dr. E. Formisano (UM)

Prof. dr. R. W. Goebel (UM)

Prof. dr. E. Stice (Oregon Research Institute, USA) 


\section{CONTENTS}

$\begin{array}{lll}\text { Chapter } 1 & \text { Introduction }\end{array}$

Chapter 2 Hunger is the best spice: an fMRI study of the effects of attention, hunger and calorie content on food reward processing in the amygdala and orbitofrontal cortex

Chapter 3 When not eating becomes easy: decreased food reward processing versus increased inhibitory self-control in currently-ill Anorexia Nervosa patients, an fMRI study

Chapter 4 Fighting food temptations: the modulating effects of cognitive reappraisal, suppression and up-regulation on mesocorticolimbic activity related to appetitive motivation

Chapter 5 Cognitions and emotions in eating disorders

Chapter 6 General Discussion

Summary

Samenvatting

References

Dankwoord 

General Introduction 
Many people believe that their eating behaviour is mainly driven by homeostatic processes (Assanand, Pinel, \& Lehman, 1998). If eating behaviour were indeed controlled by homeostasis alone, most people would only eat when in need of energy. Observations of human eating behaviour in restaurants or at parties however indicate that is not necessarily true. In fact, many people will eat when presented with highly palatable foods, independent of how hungry they are; there is always room for dessert! In the past several decades researchers have started to unravel the mechanisms that underlie this type of non-homeostatic eating behaviour, in the hope that it will provide insights into why some people eat too much and others eat too little.

In this chapter, first human eating behaviour will be discussed from a homeostatic perspective, explained why homeostasis on its own cannot sufficiently explain all occurrences of human eating behaviour. Second, the incentive motivation theory will be presented (Berridge, 2004; Bindra, 1978; Bolles, 1972; Toates, 1986). This theory proposes that food acquires a reward value through processes of associative learning, and as a consequence comes to trigger eating behaviour in the absence of biological needs. Researchers have now started to gather evidence in support of the incentive motivation theory, and have provided important insight into factors that influence eating behaviour.

A research method that has provided especially usefull insight is that of functional magnetic resonance imaging (fMRI). FMRI allows researchers to study activity within the dopaminergic mesocorticolimbic circuitry, which is theorized to be the region in the brain where incentive motivation is processed (Ikemoto, 2007; Robinson \& Berridge, 1993). FMRI has also been the primary research method in the present dissertation and therefore a brief explanation of fMRI and its application in food reward research is provided.

The study of mesocorticolimbic incentive motivation processing has also inspired researchers to develop theories on how these processes might be impaired in people with abnormal eating behaviour, like Anorexia Nervosa patients and obese people. A short review of these theories will be presented. Finally, it will be argued that the investigation of cognitive control strategies that can influence mesocorticolimbic activity might provide important insights into how to alter impaired food reward processing. This chapter concludes with an overview of the central aims and outline of the present dissertation. 


\section{EATING BEHAVIOUR: HOMEOSTASIS}

Eating and drinking behaviour is critical for human survival, providing the body with nutrients and energy. Energy homeostasis is the process by which energy stored in the body is held constant over long periods of time. For this process to occur, the amount of energy consumed must match the amount of energy expended. An error in the adjustment of food intake to energy expenditure of only $1 \%$, would lead to a body weight gain of about $1 \mathrm{~kg}$ per year, or $50 \mathrm{~kg}$ over an average adult lifespan (Shin, Zheng \& Berthoud, 2009). In most adults however, body weight is remarkably constant despite huge variations in daily food intake and energy expended, which suggests the existence of an active regulation mechanism.

Max Kleiber (1947, discussed in Keesey \& Powley, 2008) first described the consistent relationship between daily energy expenditure and body weight. Studying 26 species, Kleiber generated a function explaining the daily energy expenditure in kilocalories per day in relationship to body weight. A best fit of the resulting function yielded that as species increase in size, their daily energy needs increase at a rate 3/4 that of their body mass. Since then researchers have demonstrated that Kleiber's formula can be successfully applied across the entire animal kingdom (Keesey \& Powley, 2008), suggesting that our body can indeed actively regulate and stabilize body weight. But how does the human body achieve such a fine balance?

In his book Wisdom of the Body (1932), Walter Cannon was the first to introduce the concept homeostasis, which captures the processes involved in the maintenance of a stable internal body state. Cannon described homeostasis as "a fairly constant or steady state, maintained in many aspects of the bodily economy even when they are beset by conditions tending to disturb them" (cited in Wolfe, Barger, \& Benison, 2000, p. 152). In research, homeostasis generally refers to a specific type of regulatory system that uses a setpoint (i.e., built-in goal value) to maintain a stable physiological state. This setpoint is constantly compared to the actual physiological state at that moment, and this comparison detects whenever a mismatch occurs between the physiological state and its setpoint goal value. When a mismatch is detected, a homeostatic mechanism triggers an appropriate correctional response. Following this description of homeostasis, it can be concluded that if human eating behaviour is indeed regulated by homeostasis, 
underlying physical processes should include a setpoint, a mismatch detector and an error correction mechanism.

The last two decades have produced dramatic advances in our understanding of the homeostatic processes that are involved in maintaining a stable body weight. Together, these findings have supported the existence of homeostatic eating mechanisms by identifying neural and hormonal feedback circuits that function like a homeostatic mechanism (Murphy, Dhillo \& Bloom, 2006). These circuits can be broadly divided into short-term and long-term homeostatic feedback mechanisms. Although a full review of these mechanisms is beyond the scope of this dissertation, one short-term and one long-term homeostatic mechanism will be briefly discussed.

A short-term homeostatic feedback mechanism involved in the regulation of eating behaviour, is that involving the arcuate nucleus of the hypothalamus. This nucleus, which is not protected by the blood-brain barrier, acts as an error detector. There are two neuronal populations within the arcuate nucleus that act like a mismatch detector by monitoring setpoint values of peripheral gut hormones and producing feedback signals (e.g., correctional mechanism). The first neural population in the arcuate nucleus inhibits food intake by detecting increases in peripheral gut hormones (e.g., peptide YY, glucagon-like peptide 1 and insulin) and sending error signals via the expression of the neuropeptides pro-opiomelanocortin and cocaine- and amphetamine-regulated transcript (Kristensen et al., 1998). The other neuronal population in the arcuate nucleus stimulates food intake by detecting decreases in peripheral gut hormones and expressing neuropeptide $\mathrm{Y}$ and agouti-related peptide (Hahn, Breininger, Baskin, \& Schwartz, 1998).

A recently discovered long-term homeostatic mechanism regulating eating behaviour is that involving leptin. This hormone, secreted from fat tissue, signals the amount of stored fat in the body to neurons in the basolateral hypothalamus (Barsh \& Schwartz, 2002). Zhang and colleagues (1994) were the first to discover leptin and its function, through studying a genetic mutation in a string of obese mice, called ob/ob mice. They found that due to this genetic mutation, the fat tissue of the ob/ob mice does not synthesize leptin. As a result, the amount of stored fat is no longer effectively signalled to the basolateral hypothalamus. The basolateral hypothalamus detects minimal levels of leptin and consequently triggers eating behaviour, which in turn results in the obesity of ob/ob mice. 
Understandably, this discovery led many researchers to believe that leptin might also be implicated in the development of human obesity, resulting in a true leptin-hype. However, current findings show that the overwhelming majority of obese people have high leptin levels, corresponding with the amount of stored body fat (for a review see Bluher \& Mantzoros, 2009). In addition, very few obese people respond to exogenous leptin administration by losing weight (Heymsfield et al., 1999; Zelissen et al., 2005). Together these findings suggest that a lack of leptin is a strong homeostatic stimulator, but that an excess of leptin is not a strong inhibitor of eating behaviour.

In conclusion, in the past decades studies of human eating behaviour have been dominated by homeostasis research. Now, more than 30 hormones and 100 peptides have been found to be secreted by the gastrointestinal tract, that might somehow be involved in the regulation of eating behaviour (for a review see Keesey and Powley, 2008). An unfortunate implication of this homeostatic approach is that it has been diverting attention away from other essential questions. For example, how does the body trigger eating behaviour when there are no obvious homeostatic needs, or in other words: why is there always room for dessert?

\section{EATING BEHAVIOUR: INCENTIVE MOTIVATION THEORY}

People often eat in the absence of homeostatic deficits (Saper, Chou, \& Elmquist, 2002). This is especially evident in modern Western societies, where already overweight people often over-eat on easily available, highly palatable, highcalorie foods (Brownell, 2005). This type of overconsumption may look homeostatic, because the extra energy could benefit long-term body weight stabilization in situations when food is scarce. However, eating in the absence of clear biological needs is by definition non-homeostatic: no deficit or deviation from a setpoint is present, and therefore eating behaviour could not have been triggered by a homeostatic error correction mechanism.

In a revolutionary and daring article, the biopsychologist Robert Bolles (1980) was one of the first to propose that eating behaviour is not purely regulated by homeostatic mechanisms. He argued that human body weight settles around a point that is only moderately stable and that the recent increase in obesity could 
not be attributed to a general rise in population setpoints. Instead, he argues that changes in human eating behaviour are due to changes in environmental conditions and food palatability. In line with Bolles' proposition, nonhomeostatic theories of human eating behaviour propose that eating behaviour prevents energy deficits, rather than reacting to declines of the body's energy below a setpoint. In other words, in a well-nourished individual, eating behaviour does not typically result from energy deficits (Berridge, 2004; Booth, 1991; Toates, 1981).

Important insight into non-homeostatic mechanisms that regulate eating behaviour was provided by a series of brain stimulation studies. First, Anand and Brobeck (1951) discovered that stimulation of the lateral hypothalamus decreased eating behaviour, while lesions in the ventromedial hypothalamus increased eating behaviour. Old and Milner (1955) showed that self-stimulations of the septal area in rats led to a rewarding experience, which was stronger and preferred over the reward of food and sex. Combining these two discoveries, and in line with the homeostasis theory, Valenstein, Cox, and Kakolewski (1970) hypothesized that stimulating hypothalamic brain regions that inhibit eating in rats would produce results similar to those of regions that, when stimulated, deliver a rewarding experience: a decrease in food consumption indicates that homeostatic processes are being satisfied and thus should have a rewarding effect. Surprisingly, results showed the exact opposite. Stimulation of lateral hypothalamic brain regions led both to an increased consumption and to the experience of reward.

In line with the brain stimulation discoveries, Bolles (1972) proposed that people are motivated to eat due to incentive expectancies: the learned expectations of a hedonic reward (e.g., associative learning). In addition, he proposed that eating behaviour can be triggered by all kinds of stimuli (e.g., a yellow M) as long as they have been paired with the delivery of a hedonic food reward. In other words, according to Bolles, food stimuli motivate eating behaviour because they trigger the expectancy of a reward.

Both Bindra (1978) and Toates (1986) elaborated on Bolles' theory. Bindra (1978) suggested that when a neutral stimulus becomes associated with a food reward, it actually evokes the same motivational state normally caused by the reward itself. This proposition is in line with the classical Pavlovian theory of conditioning, which states that a neutral stimulus (conditioned stimulus) paired with a reward 
(unconditioned stimulus) becomes a reward in itself. This is nicely illustrated by the observation that when animals learn that the onset of a light (CS) predicts the arrival of a food reward (US), the animals will actually try to eat the light, a phenomenon also referred to as autoshaping (Brown \& Jenkins, 1968).

Toates (1986) rightfully noted that in its current definition, Bindra's theory (1978) predicts that one should always respond to a rewarding stimulus. Clearly, this is not true. Physiological deficits such as hunger or thirst do modulate motivation for food rewards. To incorporate homeostasis into Bolles' theory, Toates suggested that homeostatic deficits enhance the reward value of stimuli. In other words, homeostatic deficit signals do not drive motivated behaviour directly, but they do magnify the hedonic impact and reward value of food. The modulation of incentive value by homeostatic deficits is also referred to as allesthesia (Cabanac, 1979), which is nicely illustrated by the saying "Hunger is the best spice".

The propositions made by Bolles, Toates and Bindra are incorporated in the incentive motivation theory (Berridge, 2004). In conclusion, the incentive motivation theory posits that through processes of associative learning, neutral stimuli that are paired with food consumption (reward) acquire a motivational value that drives eating behaviour, even in the absence of a clear biological need. Hunger can increase the reward value of food, but by itself it is not enough to drive human eating behaviour.

\section{FOOD REWARD: GENERAL CONSIDERATIONS}

Before continuing this chapter, three general issues need to be clarified in order to promote the reader's understanding of the present discussion: 1 ) the use of the term food reward instead of incentive motivation, 2) the definition of reward, and 3 ) the measurement of food reward.

In incentive motivation research, researchers more commonly use the term food reward. The use of this term is often preferred by researchers due to the fact that term incentive motivation is not only used to explain motivated behaviour triggered by foods but also by other rewarding stimuli (e.g., drugs, sex; Robinson and Berridge, 2003). In order to refer more directly to incentive motivation related to food, researchers have more commonly adopted the term food reward. 
In addition, the term reward is preferred to the term reinforcement, because reinforcement is usually used to refer to a retroactive effect of learning, occurring after the behaviour is being reinforced. By using the more general term reward, one remains true to the incentive motivation theory, by also referring to effects that lead up to behaviour (Wise, 2004).

A second important issue that needs to be clarified is the definition of reward. Most people will probably think of a reward as something that is desired, capable of producing a conscious experience of pleasure. However, this definition of reward leads to several fundamental problems in research. For example, does this mean that reward can only be studied in those capable of verbalizing their conscious pleasurable experiences? Do animals have conscious experiences of pleasure? If not, does this imply that reward processing cannot be studied in animals? Furthermore, the generation of subjective feelings of pleasure is a key function of reward, but it is still unclear whether the pleasure itself has a reinforcing and causal effect for behaviour, or whether it is simply an epiphenomenon. In order to provide a less problematic research definition of reward, researchers take a behaviourist approach; it is suggested that reward is something that increases the frequency and intensity of a specific behaviour that has resulted in reward before (Epstein, Leddy, Temple, \& Faith, 2007; Schultz, 2006).

An important issue when defining food reward in terms of behaviour is avoiding an error in logic called circular reasoning. Circular reasoning refers to the explanation of something in terms of itself. For example, a woman is presented with two foods. She chooses one of the two foods and eats it. In line with the incentive motivation theory, it is proposed that she selected that food because it was more rewarding compared to the other. But how can it be inferred that the food was more rewarding? This conclusion is based on the fact that she ate the selected food and not the other. This type of explanation is what is referred to as circular reasoning (see Figure 1.1). Circular reasoning is unscientific, because it cannot be falsified (Popper, 1972).

To avoid the error of circular reasoning, an operational definition is adopted (Bridgman, 1982). An operational definition attempts to define a concept in terms of the way it is measured. For example, the woman picked that particular food because it contained more calories. This operational definition in terms of calorie content is falsifiable, and testable predictions can be formulated. For example, if 
food reward can be operationally defined in terms of calorie content, increasing calorie content should increase food reward and therefore increase eating behaviour.

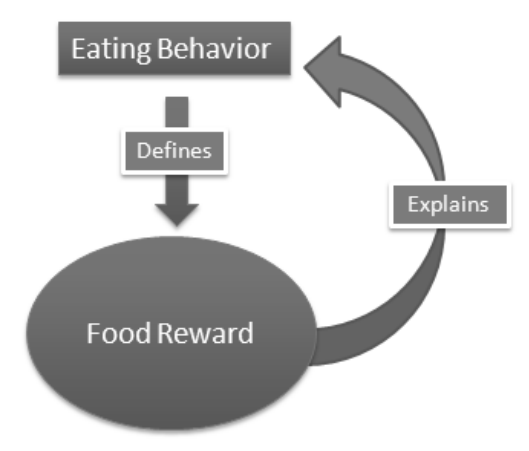

Figure 1.1 Circular reasoning in defining food reward.

The third and final issue is how food reward is measured. In line with the operational definition of food reward and the behaviouristic approach, it might be proposed that one should manipulate variables that are proposed to influence food reward, and then to investigate changes in behaviour. The most obvious method to study food reward is to interview people about their eating. For example, a researcher interested in the influence of emotions on food reward driven eating behaviour could ask the participants: "Do you feel an increased urge to eat high calorie foods when you are sad, even when you are not hungry?". Although many people are very good at indicating whether and when they like specific foods, self-report measures also have several disadvantages. First, the structure of the questions asked affects whether the reported information accurately reflects the construct under consideration. For example, asking participants to reflect on the urge to eat food might lead to several different interpretations (e.g., referring to the amount of salivation, rumbling of the stomach, amount of food-related thoughts or physical restlessness). In line with this reasoning, Schwarz (1999) concluded that self-reports are an untrustworthy source of data, because minor changes in question wording, question format, or question context can result in major changes in results. Another major problem is 
that some aspects of food reward that trigger motivated eating behaviour are proposed to be unconscious, including core liking reactions and incentive salience wanting (for a review see Berridge \& Kringelbach, 2008). Therefore, people might simply not be able to consciously reflect on certain aspects of food reward processing.

A more elegant way of investigating food reward, is by actually observing changes in intensity and/or frequency in behaviour in the laboratory. Indeed, this is how food reward is studied in animals. For example, Pellegrini and Papini (2008) measured how hard rats were willing to work to obtain sucrose water compared to freely available regular water, by changing the number of leverpresses needed to obtain sucrose. Results showed that increasing the percentage sucrose led to a similar increase in the amount of lever-presses the rats were willing to make, before switching to regular water.

This type of animal research has inspired ideas of how to study food reward in people. One can understand that it is quite awkward and unnatural to have people press levers in order to obtain food reward. Therefore researchers have adapted this lever-pressing paradigm into a more suitable computer task to study food reward in people, called the concurrent schedule paradigm (Lappalainen \& Epstein, 1990). Applying this paradigm, the number of button presses is progressively increased every time a food related reward (e.g., points they can later exchange for food) is obtained. Reward value is most commonly defined in terms of the breakpoint, the point at which the participant stops responding. A food reward that maintains a higher breakpoint is considered to be more rewarding than food for which responses are terminated earlier.

In addition to the concurrent schedule paradigm, a new and exciting research method has recently been applied in the study of food reward: functional Magnetic Resonance Imaging (fMRI; Ogawa, 1992). This method allows for the direct observation of brain activity in response to experimental manipulations, providing a very sensitive dependent variable without the necessity of overt responding. 


\section{FUNCTIONAL MAGNETIC RESONANCE IMAGING AND FOOD REWARD RESEARCH}

Food reward processes can be investigated by directly observing and measuring brain activity known to be involved in food reward processing. One method that allows for such measurements is fMRI (Ogawa, 1992). Because this has been the primary research method used in the present dissertation, a more detailed description of fMRI will follow. However, a full description of this complex research tool is beyond the scope of the present dissertation and interested readers are referred to Heuttel, Song and McCarthy (2004).

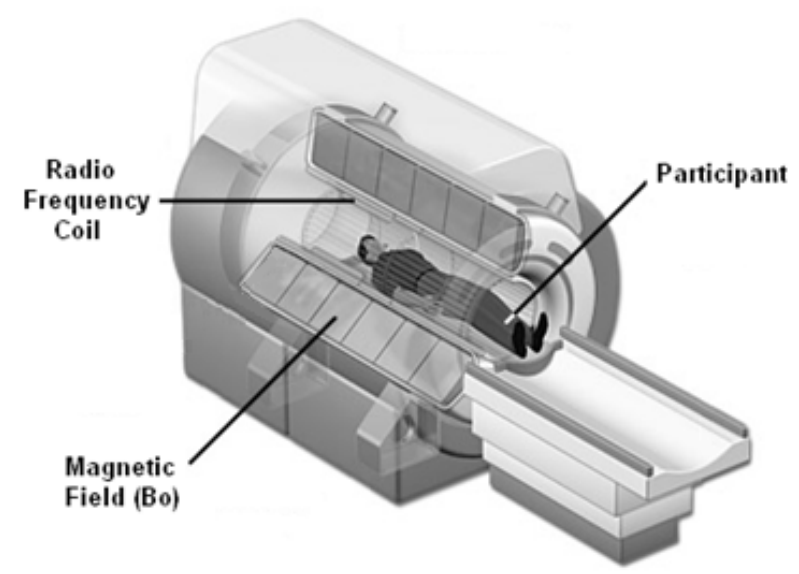

Figure 1.2 MRI scanner.

The magnetic resonance (MR) signal, measured with a magnetic resonance imaging (MRI) scanner, results from the magnetic properties of hydrogen atoms, part of the water molecules which make up $70 \%$ of the human body. Without digressing into physical details, it can be stated that by placing the human body in a strong static magnetic field (B0; Figure 1.2) the rotation axes of most hydrogen atoms become aligned to the direction of the strong magnetic field. By applying a brief pulse using the radio frequency coil, energy is added to the hydrogen atoms causing them to rotate faster. Because of the increase in rotation 
speed, the rotation axes of the hydrogen atoms are no longer aligned to the main magnetic field. When the application of the energy pulse is stopped, the main hydrogen atoms release the extra energy and return to their original state. Depending on their direct environment, the hydrogen atoms release this added energy quickly (strong MR signal) or relatively slowly (weak MR signal). In fMRI studies, the influence of the environment on the MR signal is determined by presence of oxygenated hemoglobin, part of red blood cells. When the hydrogen atom is surrounded by oxygen-rich hemoglobin, the MR signal is strong. When the direct environment contains oxygen-poor hemoglobin, the MR signal will be weak. This specific type of MR signal influenced by the presence of hemoglobin is called the T2* signal. Ogawa (1992) showed that when brain regions become active they metabolize oxygen, causing changes in the T2* MR signal according to a specific pattern. This pattern is called the blood oxygenation level-dependent response, or BOLD response. The BOLD response has proven to be a sensitive tool for mapping brain activaty and is therefore the main dependent variable in fMRI research. In other words, rather than recording the reaction times or error rates, BOLD responses are measured to determine whether there are significant changes in brain activity due to a specific experimental manipulation.

When applying $\mathrm{fMRI}$ in the study of food reward processing, a so-called functionto-structure deduction approach (Henson, 2005) is applied. The function-tostructure approach can be described as follows: if an experimental condition C1 elicits a BOLD response in brain region R1 relative to some baseline condition $\mathrm{C} 0$, and region $\mathrm{R} 1$ has been associated with function $\mathrm{F} 1$ in a different context, then function F1 is also implicated in condition C1. Applying this definition to fMRI research on food reward specifically, it is assumed that when an experimental manipulation elicits a BOLD response in a brain region that has previously been associated with reward processing, then reward processing is also implicated in the experimental manipulation. In other words, by focusing on brain regions known to be involved in food reward processing, it can be more specifically tested whether experimental conditions induce food reward activation. From this it can be concluded that in order to study food reward processing using $\mathrm{fMRI}$, one has to have knowledge about brain regions known to be involved in food reward processing. 


\section{BRAIN REGIONS INVOLVED IN FOOD REWARD PROCESSING}

The neurotransmitter dopamine is almost synonymous for reward (Wise, 2004). The focus on dopamine was triggered by a series of experiments showing that natural rewards like foods trigger dopamine transmission in the brain (Kelley \& Berridge, 2002; Salamone, Correa, Mingote, \& Weber, 2003), and that blockage or stimulation of dopamine receptors modulate reward driven behaviour (for a review see Salamone \& Correa, 2002). The observation that dopamine antagonists decrease the effort animals exert to work for a food reward led Wise (1978) to propose his famous anhedonia hypothesis. This hypothesis states that that dopamine transmission directly mediates the hedonic pleasure elicited by rewards. Although this theory has recently undergone a lot of criticism focused on the idea that dopamine is not involved in pleasure but in effort-related aspects of food motivation (Salamone, Correa, Mingote, \& Weber, 2005), dopamine has been and still is the central focus of reward researchers.

Unfortunately, dopamine signaling cannot be directly measured using fMRI, although some researchers suggest that BOLD responses can reflect dopamine transmission (D'Ardenne, McClure, Nystrom, \& Cohen, 2008). Rather than focusing on dopamine transmission directly, food reward researchers using fMRI measure BOLD responses in brain regions known to be dominated or influenced by dopamine signaling. These regions are known as the mesocorticolimbic circuitry. The mesocorticolimbic circuitry is actually a combination of two pathways, (1) the mesolimbic and the (2) mesocortical pathway (Ikemoto, 2007). The mesolimbic pathway transmits dopamine form the ventral tegmental area to the nucleus accumbens in the ventral striatum, and includes the amygdala and the hippocampus. The second pathway, the mesocortical pathway, originates in the ventral tegmental area from which connections project to the cortex, including the orbitofrontal cortex (Kringelbach, 2004), the insula (Augustine, 1996) and the anterior cingulate cortex (Paus, 2001; Figure 1.3). Together, these regions play a crucial part in reward processing and the generation of motivated behaviour.

Our brain is highly efficient, and therefore it is unlikely that all regions within the mesocorticolimbic circuitry perform the same function. Although a complete review of the functionality of each of these regions is beyond the scope of this 
introduction, the mesocorticolimbic regions and their function in reward processing are briefly discussed.
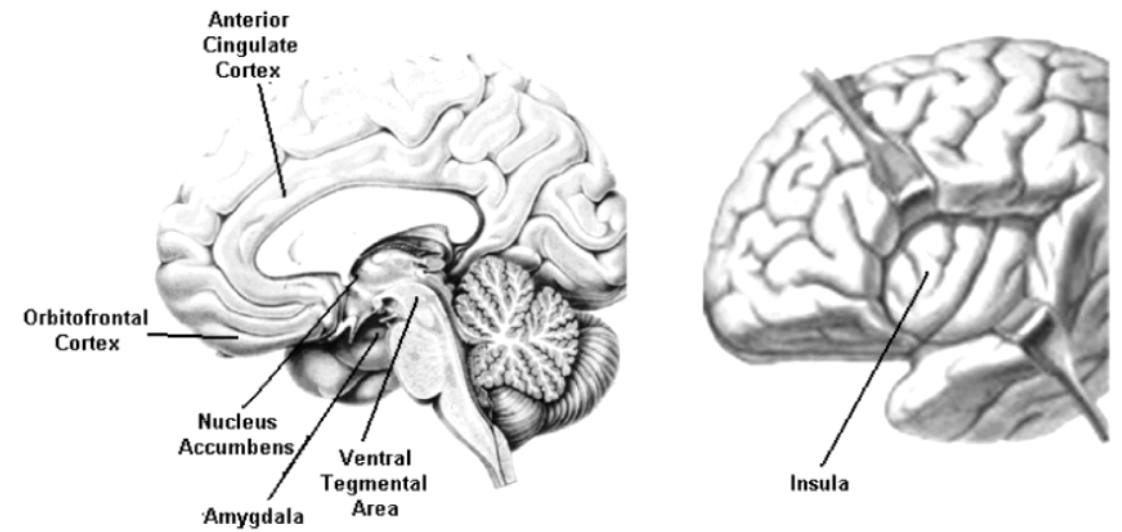

Figure 1.3 Regions of the mesocorticolimbic circuitry.

The ventral tegmental area is a group of neurons located in the midbrain, at the top of the brainstem. The neurons of the ventral tegmental area synthesize dopamine. The lateral ventral tegmental area projects to the ventrolateral striatum, including the nucleus accumbens core and shell (Ikemoto, 2007). The nucleus accumbens is a collection of neurons located at the head of the caudate and the anterior portion of the putamen. Together with the ventral tegmental area, the nucleus accumbens has been implicated in the rewarding effect of drugs and food (Wise \& Bozarth, 1987). For example, depleting dopamine within the nucleus accumbens reduces the rewarding effects as measured with instrumental responding (Caine \& Koob, 1994) and conditioned place preference (Spyraki, Fibiger, \& Phillips, 1982a, 1982b).

The amygdala is an almond-shaped group of nuclei that has been especially popular in the study of fear conditioning (Murray, 2007). Interestingly, recent research indicates that the amygdala also has a role in the generation of positive reinforcement, suggesting its more general role in representing stimulus-reward associations (for a review see Baxter \& Murray, 2002). In cooperation with the 
hippocampus, where location-reward associations are stored, these two structures are widely believed to be essential for the representation of associations between stimuli and places and their reward value (Maren, 2008).

In addition to the amygdala, the orbitofrontal cortex has also been implicated in the generation of stimulus-reward representations (Murray \& Izquierdo, 2007). In contrast to amygdalar processing, which has been hypothesized to be automatic and even unconscious (Vuilleumier, Richardson, Armony, Driver, \& Dolan, 2004; Whalen et al., 1998), it is proposed that the orbitofrontal cortex is involved in the conscious hedonic experience of reward pleasure (Kringelbach, 2005). Support for this proposition comes from fMRI studies showing that increases in sensory-specific satiety, which refers to the rapid decrease in food palatability as it is eaten to satiety, are significantly correlated to decreased activity in the medial orbitofrontal cortex (Kringelbach, O'Doherty, Rolls, \& Andrews, 2003; Rolls, 2007). Following these propositions, it can be hypothesized that functional dissociation between orbitofrontal cortex and amygdalar functioning might be possible based on differences in attention requirements. This hypothesis will be addressed in Chapter 2 of this dissertation.

The anterior cingulate cortex is located on the medial surface of the frontal lobe. In general, this region is known for its involvement in error detection and the online monitoring of behaviour (Carter et al., 1998). In relationship to reward processing, Walton et al. (2009) showed that lesions in the anterior cingulate cortex, caused a significant decrease in the consumption of rewarding foods that required hard work to obtain them but did not result in general motor deficits (i.e., slowing in lever pressing). These findings suggest that the anterior cingulate cortex is involved in the motivation of effort-related reward behaviour.

The anterior cingulate cortex is often co-activated with another region of the mesocorticolimbic circuitry, the insula. The insula was long regarded as a 'simple' sensory processing region, for example, for the processing of taste and pain (Mesulam \& Mufson, 1982a, 1982b; Mufson \& Mesulam, 1982). However, Craig (2009) recently postulated that the anterior part of the insula is involved in the general representation of interoceptive feelings, including the conscious experience of craving. This proposition is supported by the finding that smokers with damage to the anterior insula could easily quit smoking because of a decreased urge to smoke (Naqvi, Rudrauf, Damasio, \& Bechara, 2007).

Together, these findings provide strong evidence of mesocorticolimbic 
involvement in the processing of (food) reward. This type of 'fundamental' research not only provides insight into the relationship between reward processing and the function of the different brain regions involved, it also opens the door for fMRI research investigating variables that influence food reward processing in relationship to (ab)normal eating behaviour.

\section{FOOD REWARD \& ABNORMAL EATING BEHAVIOUR}

Obesity and Anorexia Nervosa have become more prevalent in recent decades, leading to increased concerns about effective treatment (Currin, Schmidt, Treasure, \& Jick, 2005; WHO, 2002). Can improved knowledge about brain reward systems help guide thinking about eating disorders? As will be discussed, recent theories of mesocorticolimbic dysfunction involved in abnormal eating behaviour propose they can.

Anorexia Nervosa is a severe psychiatric condition, occurring mainly in adolescent girls and young females (Treasure, 2007). Anorexia Nervosa is characterized by the persistent pursuit of extreme thinness and a morbid fear of fatness, accompanied by a distorted body perception (APA, 2000). The incentive motivation theory of Anorexia Nervosa (Pinel, Assanand, \& Lehman, 2000) proposes that girls who are likely to develop Anorexia Nervosa, are highly controlled, rigid and obsessive. When these girls start dieting, under great pressure from a cultural emphasis on slenderness, they are able to overcome the natural reward value of food. As a result, food no longer motivates eating behaviour, allowing Anorexia Nervosa patients to resist foods and lose weight. In conclusion, according to Pinel et al. (2000) the decrease in the reward value of food is an important factor in the development and maintenance of Anorexia Nervosa. This theory is in line with a more recent neurobiological model of Anorexia Nervosa (Kaye, Fudge, \& Paulus, 2009). According to this model, perfectionism and the obsessive and anhedonic temperament of Anorexia Nervosa patients (APA, 2000) persist in a more modest form, after recovery from Anorexia Nervosa (Wagner et al., 2006). This suggests that these characteristics are trait- rather than state-related. Kaye et al. (2009) propose that these personality traits are related to exaggerated cognitive control by the dorsolateral prefrontal cortex, and that this brain region might develop excessive inhibitory 
activity to dampen information processing through mesocorticolimbic reward pathways. Alternatively, increased activation of rule-based and analytical cognitive processes might compensate for primary deficits in mesocorticolimbic reward function, which would explain the anhedonic temperament of Anorexia Nervosa patients: "The ability to 'follow one's gut (or heart)' — that is, make effective use of interoceptive information - is impaired in individuals with AN and is consistent with the observation that individuals with Anorexia Nervosa have low comorbidity with alcohol and alcohol use disorders" (p. 581; Kaye, 2009).

Recent fMRI studies support the neurobiological model of Anorexia Nervosa (Kaye et al., 2009), showing that women recovered from Anorexia Nervosa show decreased taste processing, as indicated by decreased insula activity, compared to control women (Wagner et al., 2008). In addition, it was shown that when currently-ill food-deprived Anorexia Nervosa patients were instructed to rate food stimuli, they did not show increased activity in the left lateral orbitofrontal cortex and right anterior cingulate cortex when food-deprived, compared to healthy food-deprived controls (Santel, Baving, Krauel, Munte, \& Rotte, 2006). Furthermore, currently-ill Anorexia Nervosa patients showed increased activity in prefrontal brain regions during the performance of a behavioural response shifting task, compared to healthy controls (Zastrow et al., 2009). Taken together, these results point to the direction of mesocorticolimbic hypoactivity and prefrontal hyperactivity in Anorexia Nervosa patients, supporting the neurobiological model of Anorexia Nervosa. However, fMRI studies of Anorexia Nervosa are still few in number and future research is needed that addresses this hypothesis more directly. For example, do currently-ill Anorexia Nervosa patients show increased dorsolateral prefrontal cortex when presented with foods? Furthermore, most Anorexia Nervosa patients do still eat low calorie foods, but avoid high calorie foods. Does this imply that activity in the mesocorticolimbic circuitry depends on the calorie content of food stimuli? These hypotheses will be tested in Chapter 3 of this dissertation.

Anorexia Nervosa patients eat too little. In contrast, obese people eat too much. Although altered food reward processing in obese people will not be studied in the present dissertation, a discussion on the development of obesity does provide insight into the importance of food reward research. The increase in obesity and associated metabolic diseases over the past decade has raised the concern that, if 
not controlled, it will become the number one public health threat for the $21 \mathrm{st}$ century (Sturm, 2002). Although multiple factors contribute to this rise in obesity, the increase in the diversity of and access to palatable foods in Western societies should not be underestimated. Research has indicated that increases in food availability and variety increase the likelihood of overeating (Wardle, 2007). Some researchers now even suggest that overeating might be comparable to that of a drug or alcohol addiction (Davis \& Carter, 2009; Volkow \& Wise, 2005). Explaining addictive behaviours, the incentive sensitization theory (Robinson \& Berridge, 2001) proposes that in some individuals the repeated use of drugs produces neuroadaptations in the mesocorticolimbic circuitry, rendering it increasingly and perhaps permanently hypersensitive to drugs and drugassociated stimuli. It is specifically the sensitization of the mesocorticolimbic circuitry that transforms the motivation for drugs into excessive craving. Furthermore, it is proposed that sensitization of drug craving occurs independently of changes in neural systems that mediate the pleasurable effects of drugs. In other words, the incentive sensitization theory proposes that excessive craving for a drug can produce compulsive behaviour, even if the pleasurable effects of that drug are diminished.

As mentioned previously, the neural mechanisms for food reward and drug addictions are largely overlapping (Volkow \& Wise, 2005). Frequent intake and exposure to highly palatable foods might therefore cause similar changes in the dopamine neurocircuitry, resulting in excessive food craving. Supporting this hypothesis, neuroimaging studies have shown that obese people have a reduced number of dopamine 2 (D2) receptors compared to lean people, and that this reduction in D2 receptors is inversely related to their body mass index (Volkow, Wang, Telang, Fowler, Thanos et al., 2008; Wang et al., 2001). Furthermore, there is now considerable support from fMRI studies showing that obese people have increased responsiveness in their mesocorticolimbic circuitry when presented with palatable food stimuli (Rothemund et al., 2007; Stoeckel, Weller, Cook, Twieg, Knowlton, \& Cox, 2008). In an attempt to explain the apparent contradiction between a decreased number of D2 receptors and increased gustatory and somatosensory brain activity, it has been proposed that people at risk for obesity find food consumption more pleasurable from a sensory perspective, which may lead to greater reward expectations, overeating and D2 receptor down-regulation (for a review see Stice, Spoor, Ng \& Zald, 2009). 


\section{FOOD REWARD RESEARCH \& CLINICAL APPLICATIONS}

As previously discussed, current research findings support the idea that deficits in mesocorticolimbic food reward processing are related to abnormal eating behaviour. As a result, new therapies are being developed that target these abnormalities. For example, pharmaceutical industries have now started developing drugs that decrease activity within the mesocorticolimbic circuitry by blocking its receptors using antagonists, reducing the increased responsiveness to food rewards. Results of such drug treatments show that they are indeed effective at reducing weight. However, due to their non-specificity, they seem to affect reward processing in general, which increases the risk of adverse psychiatric events (e.g., depression, anxiety, and suicidal ideation; Astrup et al., 2007; Hu, Zhu, \& Huang, 2009). Furthermore, targeting mesocorticolimbic abnormalities with drugs might not lead to long-term changes, requiring people to commit to a lifelong medication plan. Therefore, it is necessary to find safer methods to target abnormalities in food reward processing of obese people.

Currently popular think-slim diets suggest that eating behaviour can be successfully inhibited by actively controlling the way one thinks about foods (Beck, 2007; Stephens, 2007; Werrij et al., 2010). In other words, people might be able to control mesocorticolimbic activity by actively controlling the way they think about food. This proposition is supported by the finding that instructing participants to inhibit hunger feelings during food presentation resulted in decreased activation in the amygdala, the hippocampus, the insula, the orbitofrontal cortex, and the striatum in men, but not in women (Wang et al., 2009). In the battle against Anorexia Nervosa and obesity, additional research on cognitive control strategies that can also effectively modulate mesocorticolimbic food reward processing also in women, might be especially useful. In line with this proposition, Chapter 4 presents an fMRI study in which it was investigated whether (1) cognitive control of food cravings results in a decrease in mesocorticolimbic processing similar to that of the inhibition of hunger in women, (2) whether decreased mesocorticolimbic activity is indeed accompanied by increased in dorsolateral prefrontal cortex activity, and (3) whether there are additional cognitive control strategies, besides inhibition that can modulate mesocorticolimbic activity. 


\section{RESEARCH AIMS AND OVERVIEW}

Observations of human eating behaviour show that food consumption often occurs in the absence of energy deficits. Therefore homeostatic theories cannot sufficiently explain what drives human eating behaviour. The incentive motivation theory can. This theory posits that eating behaviour is motivated by the reward value of foods. Support for the incentive motivation theory has been gathered using advanced techniques, including $\mathrm{fMRI}$, studying mesocorticolimbic activity. Current findings hint that further investigation of food reward processing will be especially insightful in understanding variables that influence human (ab)normal eating. In line with this proposition, the aim of the present dissertation is to study the influence of calorie content, hunger, attention and cognitive strategies on mesocorticolimbic food reward activity and to investigate the hypothesized decrease in mesocorticolimbic food reward processing in Anorexia Nervosa patients.

In Chapter 2, an fMRI study is presented studying the effects of calorie content, food deprivation and attention on mesocorticolimbic food reward processing in healthy women. The interest in the effect of calorie content on food reward processing was inspired by the optimal foraging theory (MacArthur \& Pianka, 1966). This theory states that organisms forage in such a way as to maximize their energy intake, which would provide evolutionary benefits. Indeed, several studies indicate that people easily overeat on high calorie foods. However, it is difficult to separate effects of calorie content and palatability, as the two go often hand in hand (Green, Wales, Lawton, \& Blundell, 2000). Therefore the first aim of this study was to investigate the influence of calorie content on food reward processing, while controlling for palatability. The second aim of this study was to examine the effect of food deprivation on food reward processing. The incentive motivation theory (Toates, 1986) suggests that although homeostatic deficit signals are not sufficient to drive motivated behaviour directly, they do magnify the hedonic impact and reward value of foods. In addition, research indicates that an experimentally controlled trial of dieting increases cravings for high calorie foods (Gilhooly et al., 2007). The third and final aim was to test whether a functional dissociation could be made between food reward activity in the amygdala and the orbitofrontal cortex, based on differences in attention requirements. Although both structures have been implicated in the 
representation of stimulus-reward associations (Baxter \& Murray, 2002; Kringelbach, 2005), considering the efficiency of the brain it is highly unlikely they perform similar operations. Addressing these three aims, healthy females were presented with high and low calorie food pictures (hypothesis 1 ) after being deprived from food for 18 hours or provided with a satiating lunch (hypothesis 2) and were instructed to evaluate their palatability. In addition, attention focus was manipulated by directing participants' attention either to the food or to a neutral stimulus aspect (hypothesis 3).

In Chapter 3, an fMRI study is presented, with the central aim of studying mesocorticolimbic food reward processing in currently-ill Anorexia Nervosa patients. The positive-incentive theory (Pinel et al., 2000) proposes that Anorexia Nervosa develops when highly controlled, rigid and obsessive adolescent females begin dieting, overcoming the natural reward value of food. This theory is in line with the recently proposed neurobiological model of Anorexia Nervosa as proposed by Kaye et al. (2009). This theory proposes that perfectionism and obsessive personality traits of Anorexia Nervosa patients are related to hyperactive cognitive control, which is related to hypoactivity in the mesocorticolimbic food reward circuitry. To test this hypothesis, the present study used the same paradigm as described in Chapter 2, instructing currently-ill Anorexia Nervosa patients to evaluate the palatability of high and low calorie foods while measuring mesocorticolimbic activity system, and comparing their responses to those of satiated and hungry healthy women.

The aim of the last fMRI study, described in Chapter 4 of this dissertation, was to investigate the effects of different types of cognitive control strategies on mesocorticolimbic food reward activity in healthy females. Recent models suggest that human eating behaviour is the result of an interaction between mesocorticolimbic food reward processing and prefrontal cognitive control (Appelhans, 2009). In line with this proposition, participants were instructed to look at the food pictures and 1) imagine the food's palatable smell, texture and taste (i.e. up-regulation); 2) immediately inhibit any thoughts concerning food palatability and/or cravings (i.e. suppression); or 3) focus on alternative meanings of the presented food cues, for example, the consequences of the food for their health (i.e. cognitive reappraisal). It was hypothesized that self-reported food cravings would be increased by the up-regulation strategy, as compared to suppression and cognitive reappraisal. Furthermore, it was hypothesized that the 
cognitive reappraisal strategy would be most successful at decreasing food cravings, as compared to suppression. In line with this hypothesis, it was expected that activity in the mesocorticolimbic food reward circuitry would be increased by the strategy of up-regulation and decreased by suppression and cognitive reappraisal. In addition, it was proposed that cognitive reappraisal would be more effective in decreasing mesocorticolimbic activity, as compared to suppression because it has been suggested that it requires less cognitive capacity (Gross \& John, 2003). Regarding recent theories of the involvement of food reward processing in Anorexia Nervosa and obesity, investigation of methods that can regulate mesocorticolimbic activity might prove to be especially insightful in the development of effective weight regulation treatments. In Chapter 5, a review is presented on the main cognitive biases and impairments in cognitive processes that are demonstrated in eating disorders. In this chapter it is also discussed whether the manipulation of cognitive biases will change eating disorder psychopathology. Some of the ideas described have been inspired by the fMRI studies presented in this dissertation, illustrating how fMRI research contributes to our understanding of eating disorders and might aid in the development of successful treatments. 
Hunger is the best spice: an fMRI study of the effects of attention, hunger and calorie content on food reward processing in the amygdala and orbitofrontal cortex 


\section{ABSTRACT}

Research indicates that dysfunctional food reward processing may contribute to pathological eating behaviour. It is widely recognized that both the amygdala and the orbitofrontal cortex (OFC) are essential parts of the brain's reward circuitry. The aims of this fMRI study were (1) to examine the effects of food deprivation and calorie content on reward processing in the amygdala and the OFC, and (2) to examine whether an explicit evaluation of foods is necessary for OFC, but not amygdalar activity. Addressing the first aim, healthy females were presented with high and low calorie food pictures while being either hungry or satiated. For the second aim, attention focus was manipulated by directing participants' attention either to the food or to a neutral aspect. This study shows that hunger interacts with the energy content of foods, modulating activity in the posterior cingulate cortex, medial OFC, insula, caudate putamen and fusiform gyrus. Results show that satiated healthy females show an increased reward processing in response to low calorie foods. Confirming our hypothesis, food deprivation increased activity following the presentation of high calorie foods, which may explain why treatments of obesity energy restricting diets often are unsuccessful. Interestingly, activity in both the amygdala and mOFC was only evident when participants explicitly evaluated foods. However, attention independent activity was found in the mPFC following the high calorie foods cues when participants where hungry. Current findings indicate that research on how attention modulates food reward processing might prove especially insightful in the study of the neural substrates of healthy and pathological eating behaviour. 
Research indicates that dysfunctional reward processing may be related to pathological eating behaviour (Davis et al., 2007; Epstein et al., 2007; Robinson \& Berridge, 2001). In an environment where food is scarce, the reward system provides evolutionary benefits, because this system allows for consumption and storage of energy in the absence of homeostatic needs (Kelley \& Berridge, 2002). However, in western societies, food is highly accessible, energy-dense, heavily advertised and inexpensive. In this environment, the reward system poses a major health risk, predisposing many people to develop obesity (E. T. Rolls, 2007; Wang et al., 2001). Therefore, a strategy for understanding inappropriate or pathological eating behaviour is to identify factors that modulate food reward processes in the human brain.

It has been widely recognized that both the amygdala and the orbitofrontal cortex (OFC) play an important role in forming representations of environmental cues and their reward value (Arana et al., 2003; De Araujo \& Rolls, 2004; Gottfried, O'Doherty, \& Dolan, 2003; Kringelbach et al., 2003; O'Doherty, Rolls, Francis, Bowtell, \& McGlone, 2001; O'Doherty et al., 2000; Small, Veldhuizen, Felsted, Mak, \& McGlone, 2008). The amygdala and the OFC are densely interconnected (Ghashghaei \& Barbas, 2002), and to carry out their reward functions they interact with other dopaminergic mesocorticolimbicstructures, including the striatum, the dopaminergic midbrain and the anterior cingulate cortex (Goldstein \& Volkow, 2002). It is hypothesized that the amygdala influences behaviour by providing a direct memory link between a stimulus and its incentive value. The amygdala then projects this information to the OFC, where this information is used to predict reward outcomes (Murray \& Izquierdo, 2007). However the exact contributions of the amygdala and OFC to reward processing in general are still unknown.

Recent neuroimaging studies show a dysfunction of the mesocorticolimbic structures of obese people, which are similar to those observed in patients suffering from drug and alcohol addiction (Volkow \& Wise, 2005). For example, it has been shown that when obese participants are exposed to food, this causes large and fast increases in mesolimbic dopamine release, similar to drug-cue elicited dopaminergic responses in drug addicts (Volkow, Wang, Telang, Fowler, Logan et al., 2008; Wang et al., 2004). Furthermore, it has been shown that both patients suffering from cocaine addiction and obese people have a reduction in mesolimbic dopamine D2 receptors (Volkow et al., 1997; Volkow, Wang, 
Telang, Fowler, Thanos et al., 2008; Wang et al., 2001). In turn this reduction in D2 receptors in obese people has been correlated with a decreased metabolism in the orbitofrontal cortex (Volkow, Wang, Telang, Fowler, Thanos et al., 2008). The similar dysfunction of the mesocorticolimbic pathways in both patients suffering from addiction and obese people indicates similarities in their underlying pathology. Therefore knowledge about factors that can modulate mesocorticolimbic functioning might be especially insightful and aid in the development of effective treatments. However, little is known about factors that modulate reward processes in the brain, especially the OFC. Due to the vicinity to air-filled frontal and sphenoidal sinuses, OFC imaging is prone to artifacts, which complicates this kind of research (Kringelbach, 2004). Recently, investigators have developed specific methods to optimize imaging of the OFC (Weiskopf, Hutton, Josephs, \& Deichmann, 2006). Using these optimized methods, the current study investigates the influence of three modulating factors of reward processing in the OFC and the amygdala: calorie content, hunger, and attention.

A first hypothesized determinant of the regulation of reward processing is the calorie content of food (Drewnowski, Maillot, \& Darmon, 2008). According to the optimal foraging theory, organisms forage in such a way as to maximize their energy intake, which would provide evolutionary benefits (MacArthur \& Pianka, 1966). Indeed, several studies indicate that people easily overeat of high calorie foods (Green et al., 2000; Prentice, 1998; B. J. Rolls, 2000). However, it is difficult to separate effects of calorie content and palatability as the two go often hand in hand. This notion is supported by studies finding little effect of calorie content on food intake, when controlling for palatability (Johnson \& Vickers, 1992; B. J. Rolls, Laster, \& Summerfelt, 1989; Yeomans, Lee, Gray, \& French, 2001). So far there has been only one neuroimaging study on the effect of calorie content on food reward processing (Killgore et al., 2003). In that fMRI study, participants had to memorize pictures of high calorie foods and low calorie foods inside the scanner for a later recollection task outside the scanner. The investigators found amygdalar activity following the presentation of the food pictures in contrast to non-food objects. However, this activity did not differ between high and low calorie foods. Furthermore, no activity in the OFC was found (but no optimizing techniques for OFC imaging were used), and differences in palatability ratings between the high and low calorie foods were 
not controlled for, which makes it hard to draw definite conclusions about the effect of calorie content per se on food reward processing.

A second factor that is hypothesized to influence reward processing is hunger. It is argued that people are motivated to eat due to the incentive salience of food, which elicits craving or 'wanting' (Berridge, 1996, 2004; Robinson \& Berridge, $2000,2003)$. However, these food reward processes are often complicated by the fact that the incentive salience of food depends, to some extent, on a cooccurring homeostatic state (E. T. Rolls, 2007). It is an old saying that hunger is the best spice, referring to the allesthesia phenomenon; that foods seem more attractive and palatable when hungry (Cabanac, 1979). Yet it is still far from clear whether, how, and where in the human brain internal homeostatic processes and reward processes interact. Neuroimaging studies have indicated that the subjective evaluation of reward is highly correlated with activity in the OFC in monkeys (Padoa-Schioppa \& Assad, 2006) and humans (Kringelbach et al., 2003). Because hunger changes the subjective evaluation of foods, it has been hypothesized that the OFC plays an important role in the integration of hunger and incentive salience. In particular, it has been shown that OFC neurons in a monkey stop responding when fed to satiety (E. T. Rolls, Murzi, Yaxley, Thorpe, \& Simpson, 1986). Recent neuroimaging research (Gottfried et al., 2003; Kringelbach et al., 2003; Small, Zatorre, Dagher, Evans, \& Jones-Gotman, 2001) has shown that feeding participants to satiety has a negative effect on the reported subjective pleasantness of the specific taste, a phenomenon referred to as sensory specific satiety (SSS). This decrease in pleasantness of food correlated highly with a reduction in activity of the OFC. However, it is currently unknown whether hunger, being the opposite of satiety, increases food reward activity in the OFC.

Furthermore, it can be hypothesized that hunger in interaction with calorie content affect food reward processes. For example, hunger might actually increase the reward value of high calorie foods more than that of low calorie foods. This idea is supported by a study of Gilhooly et al. (2007), who investigated the relationship between craved foods and a 6-month dietary energy restriction. It was found that during the energy restriction participants craved energy dense high-fat foods more than twice as much as before. It is important to further explore the relationship because energy restriction is one of the most applied strategies in the treatment of obesity (Epstein, Myers, Raynor, \& Saelens, 
1998). The increase in reward value of energy dense food might explain why obese people often ultimately fail in their attempts to lose weight after some initial success (Jeffery et al., 2000).

The third factor that is hypothesized to influence reward processing is attention. Although it has been proposed that both the amygdala and the OFC play an important role in reward evaluation, their exact contributions to reward processing remain unclear. Imaging studies of the amygdala commonly associate activation of this area with the perception of visual emotional stimuli, such as facial expressions (Bishop, Duncan, \& Lawrence, 2004; Rauch et al., 2007) or unpleasant pictures (Kensinger \& Schacter, 2006). A topic of current debate is the extent to which amygdalar responses are automatic (Pessoa, Padmala, \& Morland, 2005). Several studies report a stronger response of the amygdala during automatic emotional processing compared to explicit emotional evaluation (Vuilleumier et al., 2004). It would be of interest to test whether food reward processing in the amygdala is also automatic and independent of attention focus. In contrast, OFC responses are believed to represent the explicit evaluation of food reward (Kringelbach, 2005), highly dependent on attention focus. This might explain why previous neuroimaging studies of food reward using a passive task (i.e. a task that does not require explicit evaluation) failed to show OFC activity (Killgore et al., 2003; LaBar et al., 2001; Tataranni et al., 1999). Therefore the third objective of this study was to test whether amygdalar activity is related to the automatic processing of food cues, and whether OFC activity is related to the explicit evaluation of food.

In sum, the aims of this fMRI study were to examine the modulating effects of calorie content, hunger, and attention focus on reward processing in the amygdala and OFC of non-dieting female participants. Participants were presented with pictures of high and low calorie foods that were matched for palatability, during two fMRI sessions. Before one of the sessions, participants were offered a highly satiating lunch (containing $500 \mathrm{kcal}$ ), whereas in the other session participants were food deprived for 18 hours. It was hypothesized that high calorie foods would be more rewarding than low calorie foods, and that food deprivation would increase the reward value of food cues, especially the high calorie foods. Both effects were hypothesized to be reflected in an increase in the blood oxygenation level-dependent (BOLD) response in the amygdala and OFC. The question whether explicit evaluation of food palatability is necessary 
for OFC activity but not for amygdalar activity was addressed by manipulating the attention focus of the participants. It was hypothesized that directing the participant's attention to either the food or a neutral stimulus aspect, would result in a modulation of reward processing in the OFC but not in the amygdala. That is, food reward processing in the amygdala was expected to take place independent of attention focus.

\section{METHOD}

\section{PARTICIPANTS}

Female undergraduate students were recruited by flyers posted at Maastricht University. The students who applied for participation were invited for an interview in which height, weight, age, handedness, medication use, dietary restrictions, impulsivity, and reward responsiveness traits were assessed.

Selected participants were 12 right-handed, healthy students with a normal body weight (body mass index (BMI) between 18.5 and 25, $M=21.5, S D=1.9$ ) and not currently dieting as assessed by self-report. Participants were unrestrained eaters, scoring $<15$ on the Restraint Scale $(M=8.9, S D=2.9$; Herman, Polivy, Pliner, Threlkeld, \& Munic, 1978). On average, participants were $19.3 \pm 0.9$ $(M \pm S D)$ years old. Because food intake varies across the menstrual cycle in females (Bryant, Truesdale, \& Dye, 2006), participants were selected based on the use of Combined Oral Contraceptives (COCs). COCs inhibit the production of fertility hormones and consequently prevent increases in food intake in the premenstrual phase (Goldzieher, 1994). Exclusion criteria were screened using a questionnaire and included items about personal or first-degree family history of eating disorders, depression or other psychiatric conditions and other illnesses that required a strict eating pattern (e.g., diabetes). Because certain personality traits are thought to reflect the sensitivity of the reward system (Davis et al., 2007), participants were screened on reward responsiveness and impulsivity. All participants scored within the normative ranges of impulsivity (participants' score: $M=63.3, S D=1.4$; normative score: $M=64.2, S D=10.7$ ) as measured with the Barratt Impulsiveness Scale (Carver \& White, 1994). In addition participants scored within the normative range of reward responsiveness (participants' score: $M=17.6, S D=2.1$; normative score: $M=17.5, S D=1.4$ ) as 
measured with a subscale from the BIS/BAS scale (Spinella, 2007). Written informed consent and ethical approval were obtained of each participant before the experiment. Participants completing the study received $€ 40$ compensation.

\section{STIMULI}

In a pilot study, 90 food and 40 non-food pictures were selected as candidate stimuli from an internet database (www.istockphoto.com). Subsequently, these food pictures were rated by 15 healthy, non-dieting, female volunteers on 7-point Likert scales. The food pictures were rated on calorie content (1: very low calorie - 7: very high calorie) and palatability (1: very bad tasting -7 : very good tasting). The object pictures were evaluated on their emotional valence (1: very negative associations -7 : very positive associations). Based on these ratings, 15 food pictures rated as high in calorie content $(M=6.6, S D=0.3$; e.g., chocolate, pizza, hamburger) and 15 food pictures rated low in calorie content $[M=1.5, S D$ $=0.4$; e.g., cracker, melon, carrot; paired $t$-test calorie ratings: $t(14)=56.60, p<$ $.001]$, but judged as equally palatable $[t(14)=0.58, p=.58$; high-calorie food pictures: $M=5.3, S D=0.6$; low-calorie food pictures: $M=5.1, S D=0.6$; ] were selected for the actual study. From the object pictures 15 neutral items $(M=4.0$, $S D=0.3$; e.g. light bulb, staples, golf ball) were selected [ratings did not significantly deviate from the neutral score $4 ; t(14)=0.54, p=.60]$.

The use of pictures instead of real food is supported by prior research. First, Tiggemann and Kemps (2005) showed that the visual modality was judged as most important in the mental imagery of food craving (visual modality $39.7 \%$, gustatory $30.6 \%$ and olfaction $15.8 \%$ ). Second, using fMRI, Simmons, Martin, and Barsalou (2005) showed that food pictures activate gustatory areas for taste and reward. Finally, Kringelbach and Rolls (2004) argue that the cells in OFC respond strongest to visual modality of food stimuli.

The pictures were projected as pop-out figures on a black canvas to minimize noise input (Rainer, Augath, Trinath, \& Logothetis, 2001). Noise input is defined as all information not directly related to the relevant stimulus. Another advantage is that the combination of a pop-out figure on a black background in a dark environment (scanner bore) results in a presentation of the stimulus without environmental cues about the actual size of the presented stimulus. By using a black background, the 'naturalness' of the stimuli is not disturbed by a perceived difference in stimulus size compared to the real object size. 


\section{DESIGN AND EXPERIMENTAL TASK}

This study used a 3 (picture types: high-calorie foods, low calorie foods, neutral objects) $\times 2$ (deprivation status: satiated, $18 \mathrm{hr}$ food deprived) $\times 2$ (attention focus: object/food attended, bars attended) within-subjects design.

Following Pessoa, Padmala and Morland (2005), in the experimental task participants were shown pictures of foods and neutral objects in the centre of the screen with bars aside (Figure 1). At the beginning of each block a word was shown that indicated the task of the participants. In the foods attended blocks, participants were shown the word "taste", prompting participants to judge the palatability of the presented foods ( + : index finger, -: middle finger). The neutral objects attended blocks were preceded by the word "red" and participants were asked to indicate whether the presented objects were red (+: index finger, -: middle finger). In the bars attended blocks, the word "bars" was shown and participants had to indicate whether the bars were of similar orientations (+: index finger, -: middle finger). In the foods attended conditions participants were asked to respond to the question 'how do you like the taste of the food'. They were told that it was extremely important to vividly imagine the taste of the foods during the taste blocks, as if they were actually consuming the food. Participants were not informed about the difference in calorie content of the food pictures. Finally, participants were instructed that it was important to respond as accurately as possible.

\section{STIMULATION PROTOCOL}

During the scanning session, the food, neutral object and bar-orientation conditions of the experimental task were presented in a blocked fashion. Each block (18 s) consisted of an initial word instruction (taste, red or bars; $3000 \mathrm{~ms}$ ) and 5 stimulus trials (3000 ms each) with 5 low-calorie foods, 5 high-calorie foods, or 5 neutral objects. Each trial started with a 200 ms display of a food or object and two peripheral bars to the right and left at $6^{\circ}$ eccentricity. After this stimulus display, a white fixation cross was shown for $2800 \mathrm{~ms}$ (see Figure 2.1) during which the participant could give her response. Each $18 \mathrm{~s}$ block was followed by a fixation block of $9 \mathrm{~s}$. Participants were explicitly instructed that fixation should be maintained throughout the experiment. The brief $200 \mathrm{~ms}$ display and the positioning of the stimuli within the centre of the visual field 
eliminated the occurrence of deliberate eye saccades (Pessoa et al., 2005).

For each of three runs, block-order was fully randomized and then manually checked for repetitions. For each separate participant, the order of the trials within each block was randomized. For the bar-orientation trials, $50 \%$ of the bars were matches and $50 \%$ were non-matches. Each high calorie food, low calorie food and neutral object was once displayed with matching bars and once with non-matching bars. Each block (high calorie food and taste, low-calorie food and taste, neutral objects and red, high calorie food and bars, low-calorie food and bars, neutral object and bars) was presented six times per run. Each run thus comprised 36 blocks separated by fixation periods, and lasted 17 mins. Each session consisted of three runs and two anatomical scans. The order of the runs was balanced across participants, but was kept constant over sessions within one participant. Similarly, the order of the response hand was balanced across participants and runs, but was kept constant over sessions within one participant. The order of the two scanning sessions (food deprived vs. satiated) was also balanced across participants.

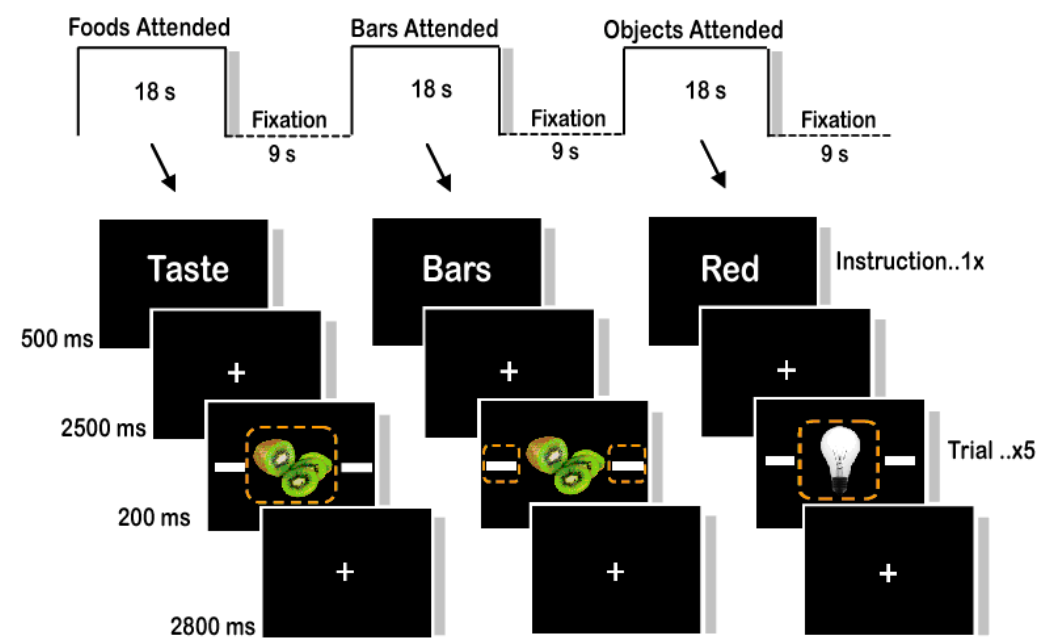

Figure 2.1 Graphical outline of the stimulation protocol used in this fMRI experiment. Stimuli in this experiment were presented in a blocked design involving three experimental conditions: foods attended (high- or low-calorie foods), bars attended and objects attended. During the tasks participants focused on the screen centre indicated by the white fixation cross. Stimuli were presented in the centre of the participants' visual field. At the beginning of each block a word was presented indicating the task and focus of attention of the participants (orange dotted line). 


\section{PROCEDURE}

Each participant underwent two fMRI sessions (food deprived and satiated), which were scheduled at least one week apart. In the food deprived condition, participants were instructed not to consume any food or beverages (except water) $18 \mathrm{hr}$ prior to the imaging session. In the satiated condition, the participant was provided with a lunch containing $500 \mathrm{kcal}$, half an hour prior to the imaging session. All imaging sessions took place around lunch-time (i.e., between 1 and 3 PM). To ensure that the food deprivation worked, prior to each session hunger was assessed with $100 \mathrm{~mm}$ visual analogue scales (VAS; translated into Dutch by Friedman, Ulrich and Mattes, 1999). The VAS questionnaire also included ratings about more general physical sensations (e.g., headache, nausea, dizziness, anxiety) to see if the food deprivation had any effects on participants' general wellbeing. Prior to entering the scanner, the participant was trained on a practice task outside the scanner, which was similar to the experimental task inside the scanner. After completing the practice task, the participant entered the scanner and completed the experimental task. Each fMRI session lasted 75 mins. At the end of the two fMRI sessions, the participant completed an exit questionnaire, inquiring about their general experience with the fMRI experiment.

\section{fMRI DATA ACQUISITION}

Images were acquired with a 3T Siemens Magnetom Allegra Head-only Scanner at the Maastricht Brain Imaging Centre (MBIC) using a birdcage volume coil. Gradient-echo planar imaging (EPI) volumes were acquired (50 slices, TR $=3000$ $\mathrm{ms})$. Imaging parameters were optimized to minimize susceptibility and distortion artifacts in OFC (Deichmann, Gottfried, Hutton, \& Turner, 2003). The relevant factors included oblique axial imaging with a negative (i.e. backward) tilt angle of 30 degrees, minimizing voxel size $(2 \times 2 \times 2.5 \mathrm{~mm})$ in the plane of the imaging, a short echo time of $25 \mathrm{~ms}$, and a high imaging bandwidth $(2790 \mathrm{~Hz}$ over the field of view, echo spacing $=0.4 \mathrm{~ms}$ ). The voxel matrix size was $128 \times 104$ and the field of view (FoV) was $256 \times 208 \mathrm{~mm}$. Acquisition of functional images yielded 340 volumes per run. Two high-resolution whole-brain anatomical T1-weighted scans were acquired: an MDEFT (Deichman, 2005; TR $=7.9 \mathrm{~ms}, \mathrm{TE}=2.4 \mathrm{~ms}$, flip angle $=15$ degrees, $1 \times 1 \times 1 \mathrm{~mm})$ an optimized MPRAGE sequence $(\mathrm{TR}=2250$ $\mathrm{ms}, \mathrm{TE}=2.6 \mathrm{~ms}$, flip angle $=9$ degrees, $1 \times 1 \times 1 \mathrm{~mm}$ ). 


\section{fMRI DATA PREPROCESSING}

All processing and analysis of the fMRI data was performed using Brainvoyager QX (v 1.9). The first two volumes of the $\mathrm{T}_{2}^{*}$ weighted functional images were discarded due to magnetic saturation effects. Preprocessing comprised slice scan timing correction (using sinc interpolation), motion correction (using a 3D rigidbody transformation of each volume to the first volume of each run and using trilinear/sinc interpolation) and high-pass filtering to remove low-frequency noise (up to 3 cycles in the single run time-course). Individual functional data were smoothed using a $6 \mathrm{~mm}$ full-width-at-half-maximum isotropic Gaussian Kernel.

For each participant, the four anatomical scans obtained throughout the experiment were averaged using a 3D rigid-body alignment to obtain a highresolution and high contrast anatomical scan. The skull and cerebellum were removed by an automatic skull stripping procedure. Functional data were averaged for each participant per condition and aligned with the mean anatomical scan.

The mean anatomical scan and the functional data were then spatially normalized using Talairach transformation procedures (Talairach \& Tournoux, 1988). For group analysis, the normalized individual functional data were averaged, accounting for both scan-to-scan and participant-to-participant variability.

\section{ANALYSIS}

Random Effects (RFX) Analysis of Variance (ANOVA) was performed to determine the effect of picture type, food deprivation and attention focus on percentage BOLD signal change per voxel. All created F-maps were thresholded at a significance level of $p<.001$ and a cluster size of 30 contiguous voxels (not corrected for multiple comparisons). Event related averaging (ERA) plots were created, visualizing the mean \% change in BOLD response in the significantly active regions of the resulting $F$-map. Functional Regions of interest (fROIs) were identified based on the hypothesis of current interest. The RFX Analysis showed that none of the voxels showed significant activity for the three-way interaction (picture type * food deprivation * attention focus). Therefore, analysis of the fMRI data is mainly based on the F-map of the two-way interaction: pictures * attention. 
To test our hypotheses, a whole brain statistical F-map was created of the RFX ANOVA F-test: picture type (low calorie food, high calorie food or neutral object) * attention focus (object/foods attended or bars attended). The resulting $F$-map revealed a network of significantly active brain regions. A brain region was indicated as fROI if significant BOLD response following the low and/or high calorie foods attended conditions, but not the neutral objects attended conditions. This allowed us to separate neutral object processing regions from food processing regions. The mean $F$-value of all voxels within each marked fROI is reported (Table 2.1) and anatomical localization was achieved using the Talairach coordinates corresponding to the centre of these fROIs. In a second level analysis, the average voxel beta values of all voxels within the identified fROls (per condition for each participant individually, resulting in a total of 144 beta's (12 participants * 12 conditions) per fROI) were extracted and submitted to a repeated measure ANOVA in SPSS. This allowed us to test the effects of calorie content and food deprivation on BOLD activity within each $\mathrm{fROI}$, especially the amygdala and OFC.

The third hypothesis concerned the functional distinction between the amygdala and OFC due to differences in attention focus. To test the first part of this hypothesis (explicit food evaluation is necessary for significant OFC activity), fROls had to be identified that responded significantly to the food conditions depending on attention focus. To achieve this goal the same RFX ANOVA F-map (pictures * attention focus) and second-level analysis were performed as for the first hypothesis. Finally, to test the second part of the third hypothesis (automatic incentive evaluation processing in the amygdala, independent of attention focus), a conjunction effects analysis was performed searching for brain activation common to all food conditions (over foods attended and bars attended conditions) versus all neutral object conditions (over neutral objects attended and bars attended conditions) in both satiated and food deprived conditions. Therefore, the conjunction test performed was food (satiated) vs. neutral (satiated) AND food (deprived) vs. neutral (deprived). The voxel beta values of fROls were extracted from each participant individually and submitted to a repeated measure ANOVA in SPSS. 
Table 2.1 Results whole brain RFX ANOVA.

F-test: Picture (neutral object vs. low and high calorie foods) $*$ attention focus (neutral object/food attended vs. bars attended)

\begin{tabular}{llcccc}
\hline Number & $\begin{array}{l}\text { Functional Region of Interest } \\
\text { (fROI) }\end{array}$ & L/R & $\begin{array}{c}\text { Talairach coordinates } \\
(\mathrm{x}, \mathrm{y}, \mathrm{z})\end{array}$ & $\mathrm{BA}$ & F-Score \\
\hline 1 & Fusiform Gyrus & $\mathrm{B}$ & $-46,-54,-13$ & 37 & 30.52 \\
2 & Basal Ganglia: $a$. Thalamus & $\mathrm{B}$ & $-12,-16,12$ & - & 26.84 \\
& $\quad \mathrm{~b}$ Ventral Striatum & $\mathrm{B}$ & $-15,-1,4$ & - & 28.31 \\
3 & Amygdala & $\mathrm{L}$ & $-13,-8,-5$ & 34 & 34.15 \\
4 & Premotor Cortex & $\mathrm{L}$ & $-3,1,54$ & 6 & 21.06 \\
5 & Inferior Frontal Gyrus, Pars Opercularis & $\mathrm{L}$ & $-46,3,31$ & 44 & 20.77 \\
6 & Anterior Cingulate Cortex & $\mathrm{L}$ & $-1,10,36$ & 24 & 21.13 \\
7 & Insula/Frontal Operculum & $\mathrm{B}$ & $-32,15,8$ & 47 & 32.33 \\
8 & Medial Orbitofrontal Cortex & $\mathrm{L}$ & $-24,29,-4$ & 11 & 31.47 \\
9 & Ventrolateral Prefrontal Cortex & $\mathrm{L}$ & $-44,36,23$ & 45 & 22.55 \\
10 & Dorsolateral Prefrontal Cortex & $\mathrm{L}$ & $-28,42,36$ & $46 / 9$ & 27.76 \\
\hline
\end{tabular}

Note: $\mathrm{L}=$ Left, $\mathrm{R}=$ Right, $\mathrm{B}=$ Bilateral, $\mathrm{BA}=$ Brodmann Area.

Unfortunately the RFX ANOVA F-test we used to analyze our fMRI data did not show any significant activity following the three-way interaction (picture type * food deprivation $*$ attention focus). Lowering the cluster size threshold or decreasing the voxel-wise significance criterion did not change this null finding. However, because effects caused by calorie content or food deprivation may be very small, our RFX analyses may have been to restrictive (type II error). Therefore in addition we applied a less strict conjunction effects analyses. A conjunction effects analysis allows for the search of significant effects following more than one t-contrast within a single voxel. Following our hypothesis of the effects of food deprivation and calorie content on reward processing, we combined two t-contrasts. First we tested for significant activation common to all food conditions (+) versus all neutral objects (-) in both food deprived and satiated attended conditions. Then we combined this $t$-contrast with a second that tested for significant interactions between food deprivation and energy content [(high calorie foods attended satiated + low calorie foods hungry) $<$ (high calorie foods attended hungry + low calorie foods satiated)]. The effects revealed by this conjunction analysis were indeed smaller compared to those of the attention manipulation. Therefore the conjunction effects t-maps were thresholded at a less strict voxel-wise significance criterion of $p<.05$ and a cluster size threshold of 10 contiguous voxels (no correction for multiple comparison). From each $t$-map fROls were identified. The average $t$-values of 
these fROIs within each map are reported (Table 2.2) and anatomical localization was achieved using the Talairach coordinates corresponding to the centre of these fROIs. The average beta weights were again extracted and submitted to a repeated measure ANOVA in SPSS.

\section{RESULTS}

\section{BEHAVIOURAL RESULTS}

Confirming that our food deprivation worked, a paired-samples t-test of the subjective hunger ratings revealed a significant difference between the hunger and satiety condition [deprived: $M=84.5, S D=7.6$; satiated: $M=12.8$, $S D=6.9 ; t(11)=20.74, p<.001]$. There were no differences between the two conditions on subjective ratings of headache, nausea or anxiety [all $p s \geq .05$, largest $t(11)=1.56$, but participants did score higher on dizziness after food deprivation than when satiated [deprived: $M=15.9, S D=13.4$; satiated: $M=3.4, S D=2.4 ; \mathrm{t}(11)=2.73, p<.05]$.

As a measure of attendance we additionally calculated the average correct response rate of the participants during all bars attended conditions. Results showed that participants responded correctly on $96.01 \%$ of the trials.

\section{FMRI RESULTS}

\section{Hypothesis I: Calorie content and reward}

The whole brain analysis of the pictures (low calorie foods, high calorie foods vs. neutral objects) * attention focus (neutral object/food attended vs. bars attended), revealed a network of areas showing significant activity. Functional regions of interest (fROIs) were identified, in which significant BOLD response following the low and high calorie foods attended conditions. Together the resulting fROIs outlined a food processing network, including the left amygdala and left medial OFC (mOFC) as shown in Figure 2.2.

The second-level analysis of the beta weights revealed that there was no significant difference in BOLD activity between the high and low calorie foods in both the food deprived and satiated attended conditions in the left amygdala, both when participants where food deprived [high-calorie foods: $M=0.1$, 
$S D=0.1$; low-calorie foods: $M=0.1, S D=0.1 ; t(11)=0.08, p=.94]$ or satiated [high-calorie foods: $M=0.1, S D=0.2$; low-calorie foods: $M=0.2, S D=0.1$; $t(11)=0.17, p=.97 \mathrm{l}$. A similar null effect for the calorie content was found in left OFC for both the food deprived [high-calorie foods: $M=0.2, S D=0.1$; lowcalorie foods: $M=0.2, S D=0.1 ; t(11)=0.34, p=.74]$ and satiated condition [high-calorie foods: $M=0.2, S D=0.2$; low-calorie foods: $M=0.2, S D=0.2$; $t(11)=0.15, p=.89]$. These non-significant effects are clearly visualized in Figure 2.3A. Further examination of difference in BOLD response between the high- and low calorie food conditions in the food deprived and satiated condition did not reveal any significant effect in any of the remaining fROIs.
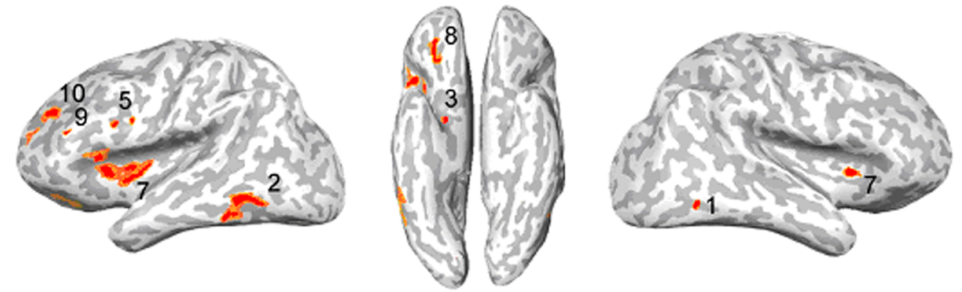

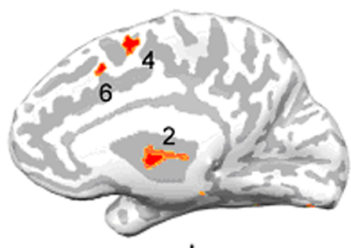

$\mathrm{L}$
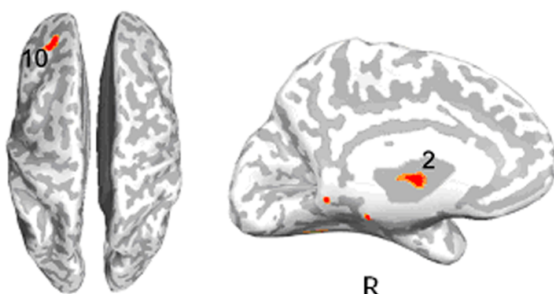

$\mathrm{R}$

Figure 2.2 Inflated brain with superimposed F-map [RFX ANOVA F-test: picture type (foods vs. neutral objects) $*$ attention focus, $p<.001$, cluster size threshold $>30$ voxels, not corrected for multiple comparisons], indicated in red all fROls (1-10) with significant BOLD activity for the pictures * attention focus interaction.

\section{Hypotheses II: Food deprivation and reward}

The second-level analysis following the RFX ANOVA F-test: picture type (low calorie food, high calorie food or neutral object) * attention focus revealed that there was no significant difference in BOLD activity between the high and low calorie foods in both the food deprived and satiated attended conditions in the left amygdala or mOFC (Figure 2.3A). The second-level Repeated Measures 
ANOVA analyses of the beta values of the remaining fROls did not show a significant effect of food deprivation on the (attended) food conditions either.

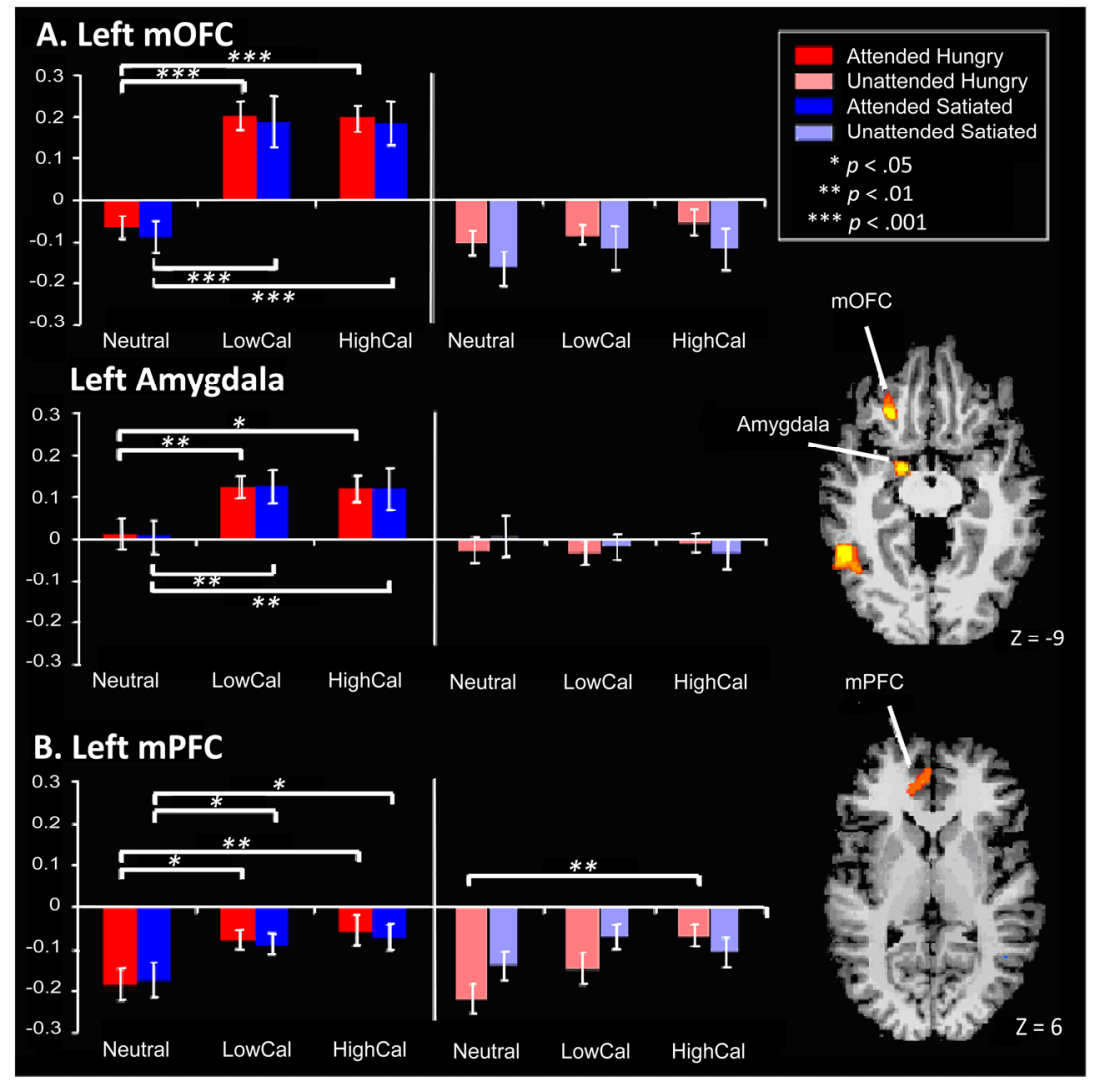

Figure 2.3 fROI bar-plots represent mean percent signal change \pm SEM. A: results of the RFX ANOVA F-test picture type (high-calorie foods, low calorie foods or neutral objects) * attention focus (satiated or $18 \mathrm{hr}$ food deprived) in the amygdala and mOFC. B: Results for the conjunction effects analysis searching for brain activation common to all food conditions independent of attention, in both satiated and food deprived conditions.

\section{Hypotheses III: Attention focus}

The whole brain statistical F-map of the RFX ANOVA F-test picture type (neutral object vs. foods averaged) * attention focus (neutral object/food attended vs. bars attended) showed a stronger BOLD response in the left MOFC in the attended foods averaged conditions (Figure 2.3A) as compared to the bars attended or 
object attended conditions. This result confirms our hypothesis that explicit evaluation of foods is necessary for OFC activity. Furthermore, other regions believed to be critically involved in food processing were also strongly modulated by attention focus (e.g., ventral striatum, premotor cortex, anterior cingulate cortex, insula/frontal operculum, ventrolateral prefrontal cortex, and dorsolateral prefrontal cortex; Figure 2.4).

Conjunction effects analysis for brain activation common to all food conditions (both foods attended and bars attended conditions) versus the neutral object conditions (both objects attended and bars attended conditions) revealed no significant effect in the amygdala (Figure 2.3A). As a result, the hypothesis that amygdala activity is independent of focus of attention was left unconfirmed. However, a significantly reduced inhibition of left medial prefrontal cortex (mPFC) activity was revealed following presentation the high calorie food conditions, independent of focus of attention focus (Figure 2.3B).

\section{ADDITIONAL CONJUNCTION EFFECTS ANALYSIS}

The whole brain analysis of the effects of food deprivation in conjunction with the interaction with calorie revealed a network of significantly active areas, including the hypothesized OFC. Other identified fROIS included the posterior cingulate cortex (PCC), insula, caudate putamen and fusiform gyrus (Table 2.2, Figure 2.4). Results showed that overall activity in these fROls was strongest for the low calorie food stimuli in the satiated conditions. Hunger increased the activity for high calorie stimuli these fROIs especially in the fusiform gyrus, right mOFC, right insula, left caudate putamen and PCC. This finding suggest that food deprivation indeed interacts with the calorie content of food stimuli and confirms our hypothesis that high calorie food stimuli become more rewarding when hungry.

Table 2.2 Results conjunction effects analyses.

\begin{tabular}{llccccc}
\hline Number & Functional Region of Interest & L/R & Tal. coordinates & BA & $t$-value & $p$-value \\
\hline 1 & Posterior Cingulate Cortex & $\mathrm{L}$ & $-6,-22,34$ & 23 & 2.73 & .004 \\
2 & Lateral Orbitofrontal cortex & $\mathrm{L}$ & $-40,36,0$ & 11 & 2.51 & .009 \\
3 & Insula & $\mathrm{R}$ & $35,3,4$ & 47 & 2.57 & .003 \\
4 & Medial Orbitofrontal Cortex & $\mathrm{L}$ & $-23,34,0$ & 11 & 2.33 & .015 \\
5 & Medial Orbitofrontal Cortex & $\mathrm{R}$ & $30,28,0$ & 11 & 2.44 & .008 \\
6 & Putamen & $\mathrm{L}$ & $-24,-8,-4$ & - & 2.45 & .008 \\
7 & Fusiform Gyrus & $\mathrm{L}$ & $-18,-49,-11$ & 37 & 2.36 & .026 \\
\hline
\end{tabular}

Note: $\mathrm{L}=$ Left, $\mathrm{R}=$ Right, $\mathrm{BA}=$ Brodmann Area. 


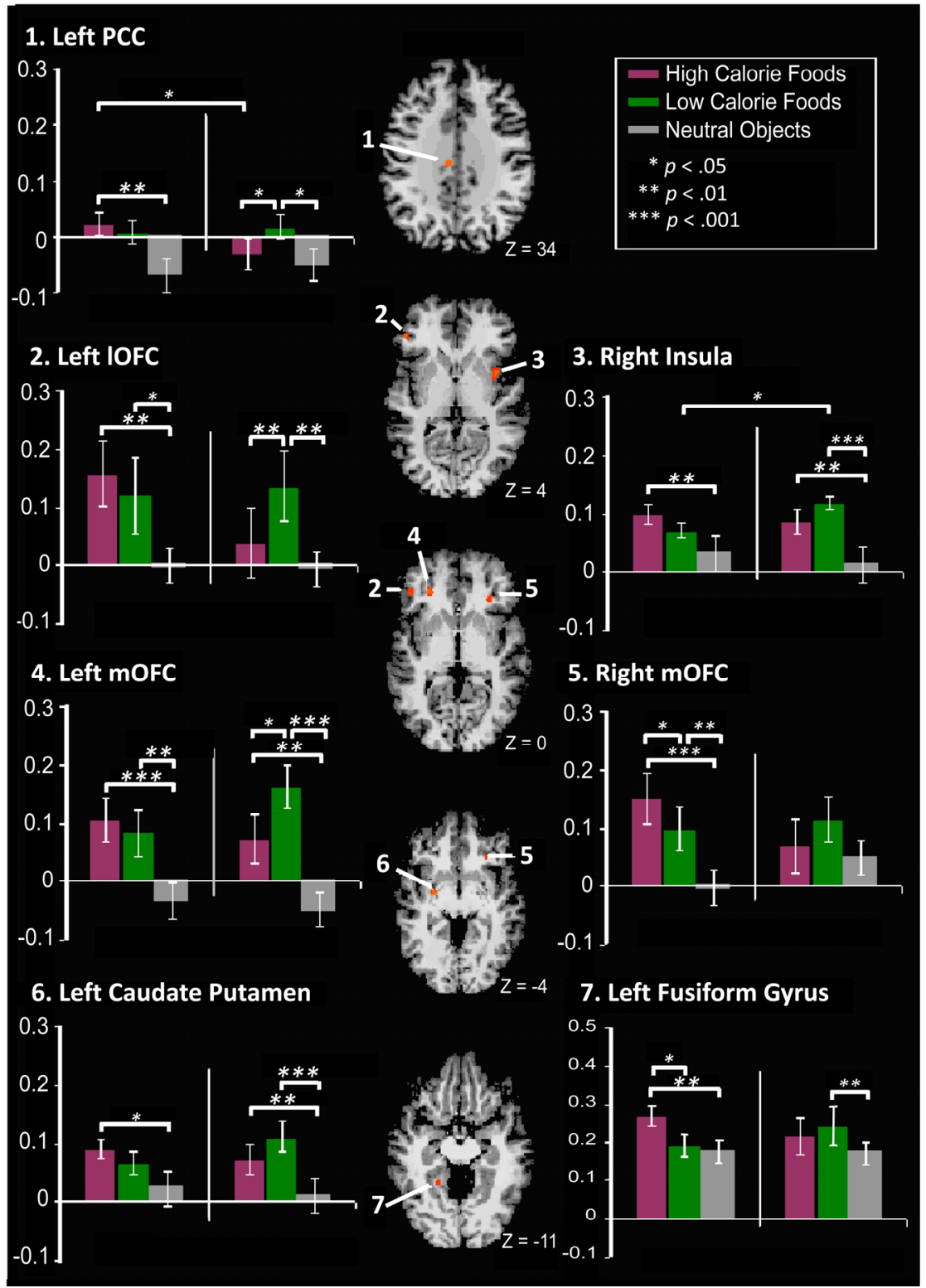

Figure 2.4 fROI bar-plots represent mean percent signal change \pm SEM of brain regions showing significant deprivation status * food calorie content interaction following the additional conjunction effects analysis. 


\section{DISCUSSION}

Although self-reports showed a successful manipulation of food deprivation on subjective ratings of hunger, the initially applied RFX ANOVA test revealed no effect of food deprivation or calorie content on reward evaluation processing in the amygdala or OFC. This suggested that hunger did not modulate the explicit reward evaluation of the high and low calorie food cues, which was a quite unexpected finding. Interestingly, a less strict conjunction analysis did reveal the hypothesized results, with significant activity in the lateral and medial OFC, PCC, caudate putamen, fusiform gyrus and the insula. Activity in these areas was strongest for the low calorie foods when the healthy females were satiated and stronger for the high calorie foods when they were hungry. The involvement of these regions in normal and abnormal reward processing is supported by several neuroimaging studies (Kable \& Glimcher, 2007; Kosten et al., 2006; Porubska, Veit, Preissl, Fritsche, \& Birbaumer, 2006; Rothemund et al., 2007; Spiegler \& Mishkin, 1981; Stoeckel et al., 2008).

The finding of a modulation by hunger and calorie content in the fusiform gyrus is in accordance with the model proposed by Murray and Izquierdo (2007), who suggest that there are two reward-processing pathways to the OFC. The first pathway concerns the reward evaluation interactions between the OFC and amygdala. The second route involves interactions between the OFC and the inferotemporal cortex, which comprises the fusiform gyrus. Murray and Izquirdo propose that this pathway allows for visual cues to elicit the predicted values of objects and is important in determining rules for future actions.

Another structure modulated by the hunger and calorie content manipulation was the right insula. Insular activity has previously been shown to be correlated with subjective cue-induced drug cravings (Bonson et al., 2002). It has also been shown that right insula activity is associated with relapse to alcohol use (Tapert, Pulido, Paulus, Schuckit, \& Burke, 2004). Furthermore, damage to the insula has been reported to disrupt addiction to smoking (Naqvi et al., 2007). It is proposed that the insula is especially involved in the conscious craving of rewards through its role in the representation of bodily states. The right insular activity found in this study might therefore reflect the participants' conscious craving or 'wanting' of the presented foods.

The finding that high calorie foods become extra rewarding when hungry has an 
important clinical implication. We previously indicated that energy restriction is one of the most applied strategies in the treatment of obesity (Epstein et al., 1998). The finding of an increased reward value of energy dense foods when hungry gives a possible reason why dietary restriction may be difficult. Diets limited in their energy content but with good satiating properties, for example high-protein diets (Clifton \& Keogh, 2007), could increase success. Furthermore, our results indicate that healthy females prefer low calorie foods when satiated. It can be hypothesized that obese people, in contrast to healthy-weight females, have increased reward processing following the presentation of high calorie foods compared to low calorie foods when satiated. This could explain part of the mechanism underlying their abnormal eating behaviour, but of course needs to be studied first.

To test the hypothesis whether an explicit evaluation of taste is necessary for OFC activity, but not amygdalar activity, we manipulated the attention focus of the participants. Results revealed a highly significant effect of attention focus on the BOLD response in several regions including the mOFC, but also the amygdala. These results suggest that explicit subjective evaluation of foods is necessary to elicit both amygdalar and mOFC activity. There was no functional dissociation between the amygdala and OFC based on differences in attention focus. The present attention effect on mOFC processing is in line with previous findings suggesting that mOFC is involved in the 'conscious' experience of reward (Gottfried et al., 2003; Kringelbach et al., 2003; Small, Zatorre, \& Jones-Gotman, 2001). However, the finding that explicit evaluation is also necessary for amygdalar processes was unpredicted. Our results suggest that amygdalar reward processing depends on attention focus and does not occur automatically. It has been suggested that studies revealing attention dependent amygdalar processing use very demanding tasks exhausting all processing capacity (Pessoa, 2005). If this is true, none of the significantly active regions should reveal an attention independent activation. However, in our study attention independent processing was found in the MPFC following presentation of high calorie foods when participants were hungry. MPFC activity has frequently been reported in fMRI studies (Killgore et al., 2003; Uher et al., 2004). However, the precise function of this region remains unclear. Previous research indicates that mPFC might be involved in self-monitoring (Amodio \& Frith, 2006) and the coordination of external versus internally generated information (Gilbert, Frith, \& Burgess, 2005). 
The attention independent activity in mPFC found in this study might represent the hungry participants' "awareness" of biologically relevant and salient high calorie foods also in the bars attended conditions. This suggestion is supported by reports in the exit questionnaires, in which participants stated that they were conscious of the presences of foods in the bars attended conditions. Therefore, our results are in line with the hypothesized role of self-monitoring and information coordination by mPFC.

Of special interest are the strong effects of the attention manipulation compared to the smaller effects of hunger and calorie content. This finding leads to several interesting hypotheses. For example, the strong effect of the attention manipulation may have been the result of our task instructions. In the food attended blocks, participants were explicitly instructed to vividly imagine the taste of the presented food pictures to evaluate their palatability. Research indicates that explicit memory processes are highly task-dependent (Allan \& Rugg, 1997) and that changes in task instructions modulate representations in working memory (Lepsien \& Nobre, 2007). In this study, to correctly perform the taste task ("How do you like the taste of this food?"), it was not necessary to actively retrieve information about the physical state of the body. This may have resulted in the weaker hunger and calorie content modulation effects, as participants could answer this question without considering their physical state. Asking participants a motivational question, for example: "How much would you like to eat this food right now?" might have yielded stronger effects. Furthermore, in this study the satiated participants were explicitly instructed to evaluate the foods, while in 'real world' situations people probably do not evaluate foods when satiated, unless they are explicitly instructed to do so. Presenting hungry and satiated participants with a passive fMRI paradigm (no explicit evaluation of food palatability) is necessary to test this post hoc explanation, as it allows participants to evaluate the foods but does not make it a task requirement. Furthermore it would be interesting to test whether obese people might frequently imagine or evaluate the palatability of foods, even when they are satiated. This would then elicit reward processes that might be experienced as craving, overruling a natural inhibition mechanism of eating behaviour.

In conclusion, robust left-hemisphere explicit food processing activity was found in the present study. Hunger and calorie content influenced reward processing in the lateral and medial OFC, cingulate cortex, caudate putamen, insula and 
fusiform gyrus. Confirming our hypothesis, satiated healthy females show a stronger BOLD response in these reward processing areas following the presentation of low calorie foods, whereas hungry healthy females showed a stronger BOLD response in these areas when presented with high calorie foods. This increase in activity is likely to represent an increase in reward value of the high calorie foods and may explain why treatments of obesity energy restricting diets are often unsuccessful. Our study highlighted the importance of attention focus in food reward processing, showing strong BOLD activity in the amygdala and mOFC when participants attended the foods, but no activity at all if the task did not require the evaluation of the foods. Attention independent processing was found in the mPFC following the presentation of high calorie foods when participants were hungry, an area proposed to be involved in linking reward to actions. These results suggest that a further investigation of attentional processes on the modulation of reward processing might be especially informative in determining the neural substrates of healthy and pathological eating behaviour. 
When not eating becomes easy: decreased food reward processing versus increased inhibitory self-control in currently-ill Anorexia Nervosa patients, an fMRI study 


\section{ABSTRACT}

Anorexia Nervosa (AN) patients starve themselves in a world of plenty. Whereas most people struggle to restrain their food intake in the present 'toxic' environment, emaciated AN patients are successful ascetics who appear to easily refuse the widely available tasty temptations. Why are they so successful in not eating? The present functional magnetic resonance imaging (fMRI) study tested the hypothesized impaired food reward processing in AN patients. Participants were AN patients of the restrictive subtype and healthy control participants. When food cues were attended, reduced activity was found in a network of brain regions including the mesocorticolimbic regions the anterior insular cortex and the caudal anterior cingulate cortex. Interestingly, when food cues were not attended, food reward processing was increased comparable to that of hungry participants and inhibitory self-control processing was increased as indicated by dorsolateral prefrontal cortex and anterior temporal cortex activity. These findings elucidate why not eating is relatively easy in AN: when paying attention to food cues, reward processing is decreased and prefrontal correction is not necessary. However, when food cues are unattended, they do elicit reward processing, meaning that $\mathrm{AN}$ patients still are responsive to food temptations. But when the AN food rewarding circuitry responds to temptation, inhibitory fronto-temporal activity is also immediately increased. This combination of increased reward processing and increased inhibitory activity might enable to resist temptations 
Whereas many people struggle to lose weight, Anorexia Nervosa (AN) patients are capable of restricting their food intake to extremes. Because the reward value of food plays an essential role in the initiation of food consumption (Berridge, 2004; Bindra, 1978; Bolles, 1972; Toates, 1986), impaired reward processing is suggested to explain why AN patients are capable of emaciating themselves (Kaye, Fudge \& Paulus, 2009; Pinel, Assenand \& Lehman, 2000). However, impaired food reward processing seems contradictive with the clinical observation that AN patients typically are preoccupied with food (e.g., dreaming of food, ruminating about food, hoarding, cooking, and stealing foods; Frankenburg, 1984; Schmidt \& Treasure, 2006). Such a preoccupation suggests intact reward processing, but even that foods are highly rewarding for emaciated AN patients.

This study investigates the hypothesized impaired food reward processing in AN patients, by measuring whole brain using functional magnetic resonance imaging (fMRI). In the first condition, AN patients and healthy satiated and hungry controls to focus at pictures of high and low calorie foods, and to imagine the food's taste. Previous research (Siep et al., 2009) indicated that hunger increases the mesocorticolimbic processing of high calorie stimuli, while satiation increases the mesocorticolimbic processing of low calorie food stimuli in healthy participants. It is expected that, even though AN patients have huge homeostatic deficits, mesocorticolimbic reward activity will be decreased comparable to that of satiated participants.

In the second condition, participants are instructed to direct their attention to a neutral stimulus, while simultaneously presting low and high calorie foods. Research indicates that the neural substrates of implicit and explicit neural processes are distinct and play diverse roles in the development and maintenance of psychopathological disorders (Lane, 2008). Therefore it can be suggested that not explicitly evaluating presented foods might result in relatively normal reward processing in AN patients, explaining the contradictive behaviour of 'not eating but preaoccupied with foods'. It was hypothesized, as opposed to the first hypothesis, that directing the participants' attention away from a food stimulus would increase reward processing in AN patients comparable to that of hungry, but not satiated control participants.

Lastly, anemia is a frequent complication of AN (Cleary, Gaudiani, \& Mehler), resulting in decreased haemoglobin levels. Because the blood oxygenation level- 
dependent (BOLD) response depends on the amount of blood haemoglobin present, a lack of haemoglobin could decrease the BOLD response and form a possible confounder in studying decreased reward processing. To investigate this, two neutral conditions were added. In the first neutral condition objects were evaluated based on their colour, in the second neutral condition objects were presented while directing participants' attention to a neutral stimulus. It is hypothesized that these conditions should not result in differences in neural activity between AN patients and healthy controls.

\section{METHOD}

\section{PARTICIPANTS}

The AN group included 12 female AN patients of the restrictive subtype (Age: $M$ $=22.3, S D=3.5$; BMI: $M=15.4, S D=1.2$ ). They were currently in treatment and had been diagnosed as AN-restricter by the intake psychologists of the eating disorder unit of the local Academic Hospital. The AN diagnosis was confirmed by the first author using the Dutch version of the Mini International Neuropsychiatric Interview (Overbeek, Schruers, \& Griez, 1999). The control group consisted of 12 healthy-weight, non-dieting female undergraduate students (Age: $M=19.3, S D=0.9 ; B M I: M=21.4, S D=1.2$ ). We previously published data of these control participants, testing the effects of hunger versus satiation (Siep et al., 2009). The medical ethical committee of the academic hospital Maastricht, The Netherlands, approved the study.

\section{EXPERIMENTAL TASK}

Stimuli and experimental task were the same as were used in previous research in our laboratory (Siep et al., 2009). The stimuli were pictures of high-calorie foods, low-calorie foods or objects presented in the centre of the screen with bars aside. At the beginning of each block a single word indicated the instruction. The food taste attended blocks were preceded by the word "taste", prompting the participant to judge the palatability of the presented foods and to vividly imagine the taste, smell and texture of the foods, as if she was actually consuming the food. No information was given about the calorie content of the foods. The neutral objects color attended blocks were preceded by the word "red", 
prompting the participant to indicate whether the presented objects were red. The bars attended blocks were preceded by the word "bars", prompting the participant to direct her attention to the bar stimuli, and to indicate whether the bars were of similar orientations.

\section{DESIGN \& ANALYSES}

Two whole-brain, voxel-wise Random Effects (RFX; 3 (picture type: high calorie foods, low calorie foods, neutral objects) $* 3$ (group: AN, hungry control, satiated control)) ANOVAs were performed, one for the taste attended condition and one for the bars attended condition. The resulting $F$-maps of the picture type $\mathrm{x}$ group interaction were thresholded at a significance level of $p<.01$. To correct for multiple comparisons, a minimum cluster size of 9 contiguous voxels was adopted (determined by a clustersize treshold estimator plugin implemented in BrainVoyager), yielding a whole-brain corrected statistical threshold of $\alpha<.05$. A functional region of interest (fROI) approach (Saxe, Brett, \& Kanwisher, 2006) was applied, which involves first identifying regions from the corrected $F$-maps that resulted from the whole brain RFX ANOVAs, and calculating the overall summary measure of response. To get more detailed insight into the effect of participants group per stimulus type within each fROI, additional one-way between-subject ANOVAs were performed. T-tests comparing the results of the AN group to the hungry and the satiated control group were performed, to follow-up significant results from the ANOVAs and to test our hypothesis. The response profiles of all fROls were visualized in bar-plots (Figure 3.2).

\section{STIMULATION PROTOCOL}

The six conditions (high calorie food and taste, low-calorie food and taste, neutral objects and red, high calorie food and bars, low-calorie food and bars, neutral object and bars) were presented in a blocked fashion. Each block (18 s) consisted of an initial word instruction (taste, red or bars; $3000 \mathrm{~ms}$ ) and 5 stimulus trials ( $3000 \mathrm{~ms}$ each) with 5 low calorie foods, 5 high calorie foods, or 5 neutral objects. Each trial started with a $200 \mathrm{~ms}$ display of a food or object and two peripheral bars to the right and left at $6^{\circ}$ eccentricity. The brief $200 \mathrm{~ms}$ display and the positioning of the stimuli within the centre of the visual field eliminated the occurrence of deliberate eye saccades (Pessoa, Padmala, \& 
Morland, 2005). After this stimulus display, a white fixation cross was shown for $2800 \mathrm{~ms}$, during which the participant responded. A fixation block of $9 \mathrm{~s}$ followed each $18 \mathrm{~s}$ stimulus block. The participant was instructed to maintain fixation throughout the experiment.

For each of three runs, block-order was pseudo-randomized. For each individual participant, the order of the trials within each block was pseudo-randomized. For the bar-orientation trials, $50 \%$ of the bars were matches and $50 \%$ were nonmatches. Each high calorie food item, low calorie food item and neutral object was once displayed with matching bars and once with non-matching bars. Each block was presented six times per run. Each run thus comprised 36 blocks separated by fixation periods, and lasted $17 \mathrm{~min}$. Each session consisted of three runs and two anatomical scans. The order of the runs and the response hand were balanced across participants.

\section{PROCEDURE}

All imaging sessions took place between noon and $3 \mathrm{pm}$. The AN patient first completed a practice task in a dummy scanner to minimize anxiety and for task practice. Control participant completed the same practice task outside the scanner on a computer. After completing the practice task, the participant entered the scanner and completed the experimental task. Each fMRI session lasted 75 mins. At the end of the fMRI session(s), the participant completed a stimulus evaluation task outside the scanner in which the food stimuli were rated on 7-point Likert scales on palatability (1: very bad tasting -7 : very good tasting) and calorie content (1: very low-caloric -7 : very high-caloric).

\section{fMRI DATA ACQUISITION}

Images were acquired with a 3T Siemens Magnetom Allegra head-only scanner at the Maastricht Brain Imaging Centre using a birdcage volume coil. Gradient-echo planar imaging volumes were acquired $(50$ slices, $\mathrm{TR}=3000 \mathrm{~ms}$, flip angle $=$ $90^{\circ}$ ). Imaging parameters were optimized to minimize susceptibility and distortion artefacts in the OFC (Weiskopf, Hutton, Josephs, \& Deichmann, 2006). The relevant factors included oblique axial imaging with a negative (i.e. backward) tilt angle of $30^{\circ}$, minimizing voxel size $(2 \times 2 \times 2.5 \mathrm{~mm})$ in the plane of imaging, a short echo time of $25 \mathrm{~ms}$, and a high imaging bandwidth $(2790 \mathrm{~Hz}$ 
over the field of view, echo spacing $=0.4 \mathrm{~ms}$ ). The voxel matrix size was $128 \mathrm{x}$ 104 and the field of view (FoV) was $256 \times 208 \mathrm{~mm}$. Acquisition of functional images yielded 340 volumes per run. A whole-brain anatomical T1-weighted scan was acquired $\left(\mathrm{TR}=2250 \mathrm{~ms}, \mathrm{TE}=2.6 \mathrm{~ms}\right.$, flip angle $\left.=90^{\circ}, 1 \times 1 \times 1 \mathrm{~mm}\right)$.

\section{fMRI DATA PREPROCESSING}

All processing and analyses of the $\mathrm{fMRI}$ data were performed using BrainVoyager QX (Brain Innovation, Maastricht, The Netherlands). The first two volumes of the T2* weighted functional images were discarded due to magnetic saturation effects. Preprocessing comprised slice scan timing correction, motion correction and high-pass filtering to remove low-frequency noise. Individual functional data were smoothed using a $6 \mathrm{~mm}$ full-width-at-half-maximum isotropic Gaussian Kernel. The anatomical scan spatially normalized using Talairach transformation procedures (Talairach \& Tournoux, 1988), together with the functional data. For group analysis, the normalized individual functional data were averaged, accounting for both scan-to-scan and participant-to-participant variability.

\section{RESULTS}

\section{STIMULUS CHECK}

No significant effects emerged in the 2 (food type: high caloric, low caloric) * 23 (group: AN, controls) ANOVA for the palatability ratings, which shows that high calorie and low calorie foods were evaluated as equally palatable [all $F(1,22)$ $<2.39$, all $p>.13$ ]. Calorie content ratings were analyzed in the same $2 * 2$ ANOVA. A significant main effect of stimulus type emerged, $F(1,22)=236.71$, $p<.001$, but there was no significant stimulus type * group interaction, $F(1,22)=$ $0.56, p=.56$ and no main effect of group, $F(1,22)=0.15, p=.71$ confirming that the high calorie food stimuli were correctly perceived as containing more calories than were the low calorie food stimuli across groups. See Table 3.1 for means and standard deviations.

As a measure of attendance, the average correct response rate during the bar attended conditions was calculated. Results showed that both groups performed equally well on the bar task (AN: $97 \%$ correct responses; control group hungry: 
$96 \%$, control group satiated: $97 \% ; F(2,33)=1.31, p=.27)$.

Table 3.1 Results stimuli ratings.

\begin{tabular}{lcccccc}
\hline & Palatability & $\begin{array}{c}\text { AN patients } \\
\text { Calorie }\end{array}$ & Affect & Palatability & Colorie & Calticipants \\
content & & & & content & Affect \\
\hline High Calorie Foods & $4.7 \pm 0.9$ & $6.1 \pm 0.8$ & - & $5.4 \pm 0.8$ & $6.3 \pm 0.3$ & - \\
Low Calorie Foods & $5.1 \pm 0.6$ & $2.4 \pm 1.0$ & - & $5.0 \pm 0.9$ & $2.1 \pm 0.5$ & - \\
\hline
\end{tabular}

Note: Numbers indicate $M \pm S D$

\section{fMRI RESULTS}

The whole brain analysis of the stimulus $\mathrm{x}$ group interaction for the taste attended conditions revealed significant effects in network of 12 brain regions (Table 3.2A, Figure 3.1), 4 of which are considered to be part of the mesocorticolimbic circuitry: the right anterior orbitofrontal cortex (aOFC; Kringelbach, 2004), the left and right anterior insula cortex (AIC; Craig, 2009) and the bilateral caudal anterior cingulate cortex (cACC; Paus, 2001). The stimulus $x$ group interaction was also significant in the left dorsolateral prefrontal cortex (dIPFC), the left premotor cortex, the left ventromedial prefrontal cortex (vmPFC), the left and right parietal cortex, the frontal eyefields (FEF) and bilateral supplemental motor area (SMA).

The whole brain analysis of the stimulus $\mathrm{x}$ group interaction for the bars attended conditions revealed significant effects in a network of 5 brain regions (Table 3.2B, Figure 3.2), 2 of which are involved in reward processing: the bilateral fusiform gyrus (FG; Geday, Gjedde, Boldsen, \& Kupers, 2003; laria, Fox, Waite, Aharon, \& Barton, 2008; Pujol et al., 2009). The stimulus $x$ group effect was also significant in the left anterior temporal cortex, right middle temporal gyrus and right dIPFC. See Table 3.3, Figure 3.1 and Figure 3.2 for a complete overview of all relevant statistics and $p$-values of the discussed $t$-tests..

For the high calorie food taste attended condition, fROI analyses showed significant effects of group were found in the left AIC, the left dIPFC, the left vmPFC, the left premotor cortex, bilateral parietal cortex (precuneus) and the bilateral CACC, left FEF and left SMA. In line with the hypothesis of deceased reward processing, pairwise comparisons showed that the group effect in the left AIC was caused by a decreased activity in the AN group compared to the hungry, but not the satiated control participants. Not in line with our hypothesis, the 
bilateral cACC showed decreased activity for the AN group compared to both hungry and satiated control participants.

Table 3.2 Resulting brain region from whole brain RFX ANOVA, F-test: Stimulus type x Group.

\begin{tabular}{|c|c|c|c|c|c|}
\hline Functional Region of Interest & $\mathrm{L} / \mathrm{R}$ & $\begin{array}{l}\text { Tal. coordinates } \\
(x, y, z)\end{array}$ & $\mathrm{BA}$ & $F$-score & $p$-value \\
\hline \multicolumn{6}{|c|}{$\begin{array}{l}\text { Hypothesis 1, Foods and Objects Attended } \\
\text { Conditions }\end{array}$} \\
\hline Anterior Orbitofrontal Cortex & $\mathrm{R}$ & $\begin{array}{lll}11 & 43 & -8\end{array}$ & 11 & 4.81 & .002 \\
\hline \multirow[t]{2}{*}{ Anterior Insula } & $\mathrm{L}$ & $\begin{array}{lll}-50 & 9 & 1\end{array}$ & 14 & 4.16 & .005 \\
\hline & $\mathrm{R}$ & $42 \quad 10 \quad 5$ & 14 & 4.80 & .002 \\
\hline Dorsolateral Prefrontal Cortex & $\mathrm{L}$ & -483625 & 46 & 5.75 & $<.001$ \\
\hline Ventrolateral Prefrontal Cortex & $\mathrm{L}$ & $\begin{array}{lll}-53 & -2 & 19\end{array}$ & 44 & 6.61 & $<.001$ \\
\hline Premotor Cortex & $\mathrm{L}$ & $\begin{array}{lll}-45 & -6 & 37\end{array}$ & 6 & 9.55 & $<.001$ \\
\hline Parietal Cortex, Supramarginal Gyrus & $\mathrm{L}$ & $-58-3539$ & 40 & 5.15 & .001 \\
\hline Parietal Cortex, Precuneus & $\begin{array}{l}L \\
R\end{array}$ & $\begin{array}{lll}-20 & -67 & 38 \\
34 & -73 & 33\end{array}$ & $\begin{array}{l}7 \\
7\end{array}$ & 5.55 & .001 \\
\hline Caudal Anterior Cingulate Cortex & B & $\begin{array}{lll}-1 & 0 & 37\end{array}$ & 24 & 5.40 & .001 \\
\hline Frontal Eye Fields & $\mathrm{L}$ & $-28-10 \quad 43$ & 6 & 4.88 & .002 \\
\hline Supplemental Motor Area & B & -2053 & 6 & 4.86 & .002 \\
\hline \multicolumn{6}{|l|}{ Hypothesis 2, Bars Attended Conditions } \\
\hline Anterior Temporal Cortex & $\mathrm{L}$ & $17-4-24$ & 20 & 4.32 & .004 \\
\hline \multirow[t]{2}{*}{ Fusiform Gyrus } & $\mathrm{L}$ & $-27-60-15$ & 19 & 4.54 & .003 \\
\hline & $\mathrm{R}$ & $25-73-12$ & 19 & 3.69 & .009 \\
\hline Middle Temporal Cortex & $\mathrm{R}$ & $45-49-11$ & 37 & 10.60 & $<.001$ \\
\hline Dorsolateral Prefrontal Cortex & $\mathrm{R}$ & $44 \quad 13 \quad 36$ & 9 & 4.72 & .002 \\
\hline
\end{tabular}

Note: $\mathrm{L}=$ Left, $\mathrm{R}=$ Right, $\mathrm{B}=$ Bilateral, $\mathrm{BA}=$ Brodmann area

\section{Hypothesis 1: decreased reward activity during food taste evaluation in AN patients, comparable to hungry but not satiated controls.}

For the low calorie food taste attended condition, significant effects of group were found in all fROIs. Not in line woth our hypothesis, pairwise comparisons showed that the AN group had decreased activity in the left and right AIC and bilateral CACC compared to both the hungry and satiated control participants. Surprisingly, the AN group showed increased activity in the right aOFC compared to both hungry and satiated control participants.

In addition to these findings, results showed that in both the low and high calorie food taste attended condition the AN patients uniquely activated the right parietal cortex compared to both the hungry and satiated control group. 

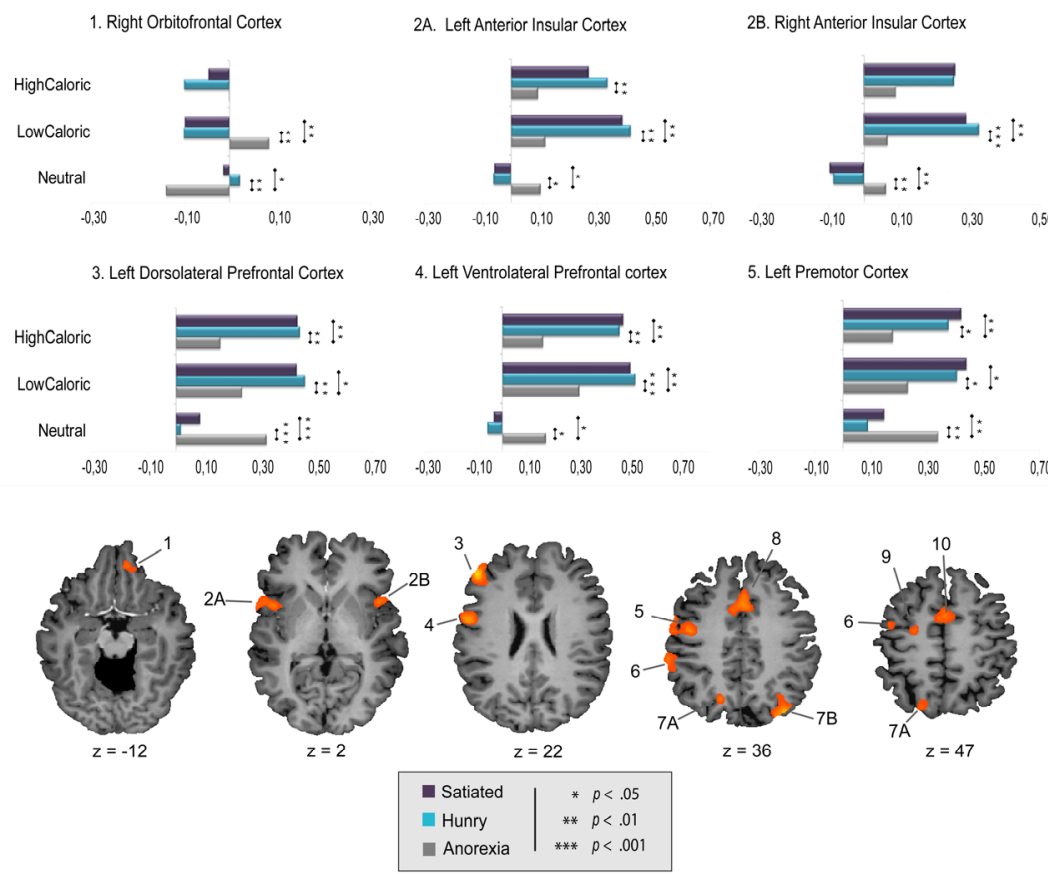

6. Left Parietal Cortex, Supramarginal Gyrus 7A. Left Parietal Cortex, Precuneus $\quad$ 7B. Right Parietal Cortex
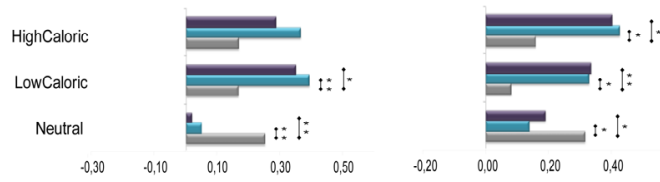

8. Bilateral Caudal Anterior Cingulate Cortex $\quad$ 9. Left Frontal Eye Fields
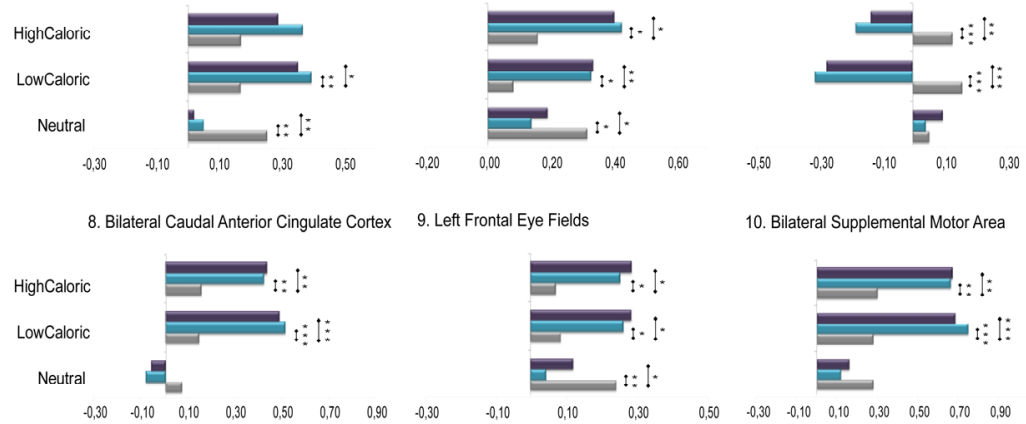

10. Bilateral Supplemental Motor Area

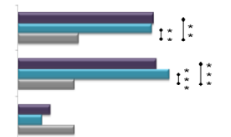

Figure 3.1 Results whole brain analyses of the stimulus $\mathrm{x}$ group interaction for the taste and object colour attended conditions. Bar-plots represent mean BOLD signal change in z-scores. 
Table 3.3 Results one-way ANOVA, F-test: group.

\begin{tabular}{lcccccc}
\hline Functional Region of Interest & \multicolumn{3}{c}{ Neutral Objects } & \multicolumn{2}{c}{ Low Calorie } & \multicolumn{2}{c}{ High Calorie } \\
& $F(2)$ & $p$ & $F(2)$ & $p$ & $F(2)$ & $p$ \\
\hline Hypothesis 1, Foods and Objects Attended & & & & & & \\
Conditions & & & & & & \\
1. Orbitofrontal Cortex & 4.43 & .02 & 8.53 & .001 & 1.56 & .22 \\
2A. Left Anterior Insula & 4.14 & .03 & 8.49 & .001 & 4.15 & .03 \\
2B. Right Anterior Insula & 5.52 & .01 & 9.66 & $<.001$ & 2.96 & .07 \\
3. Dorsolateral Prefrontal Cortex & 3.47 & .04 & 9.79 & $<.001$ & 8.99 & .001 \\
4. Ventrolateral Prefrontal Cortex & 2.67 & $<.001$ & 4.89 & .01 & 5.68 & .01 \\
5. Premotor Cortex & 7.89 & .002 & 3.67 & .04 & 4.62 & .02 \\
6. Parietal Cortex, Supramarginal Gyrus & 8.09 & .001 & 4.74 & .02 & 1.90 & .17 \\
7A. Left Parietal Cortex, Precuneus & 4.55 & .02 & 4.95 & .01 & 4.12 & .03 \\
7B. Right Parietal Cortex, Precuneus & 0.48 & .62 & 28.03 & $<.001$ & 11.44 & $<.001$ \\
8. Caudal Anterior Cingulate Cortex & 3.23 & .05 & 10.20 & $<.001$ & 7.05 & .003 \\
Frontal Eye Fields & 6.96 & .003 & 4.41 & .02 & 5.70 & .007 \\
Supplemental Motor Area & 2.85 & .07 & 12.59 & $<.001$ & 8.59 & .001 \\
Hypothesis 2, Bars Attended Conditions & & & & & & \\
& & & & & \\
Anterior Temporal Cortex & $F(2)$ & $p$ & $F(2)$ & $p$ & $F(2)$ & $p$ \\
2A. Left Fusiform Gyrus & 4.21 & .02 & 0.06 & .94 & 6.30 & .004 \\
2B. Right Fusiform Gyrus & 1.06 & .36 & 5.01 & .01 & 4.98 & .01 \\
Middle Temporal Cortex & 2.89 & .07 & 3.01 & .06 & 2.49 & .07 \\
Dorsolateral Prefrontal Cortex & 3.47 & .04 & 3.94 & .03 & 11.77 & $<.001$ \\
\hline
\end{tabular}

Note: non-significant effects are printed in Italics.

\section{Hypothesis 2: implicit processing of food stimuli will lead to increased reward processing, comparable to that of healthy hungry controls}

For the high calorie food bars attended condition, significant effects of group were found in the left anterior temporal cortex (aTemp), the right middle temporal cortex (mTemp), the left FG and the right dIPFC. In line with the hypothesis of increased reward processing, pairwise comparisons showed that the group effect in the left FG was caused by an increased activity in the AN group compared to the satiated but not the hungry control participants. This response profile was similar to that of the right mTemp. Interestingly, the left aTemp and right dIPFC showed increased activity in the AN group compared to both the satiated and hungry control participants

Similar to the effects found for the high calorie food bars attended condition, in the low calorie food bars attended condition significant effects of group were found in the left FG and the right mTemp. In line with our hypothesis, pairwise comparisons showed that the group effect in the left FG was caused by an 
increased activity in the AN group compared to the satiated group only. Again, this response profile was similar to that of the mTempC.

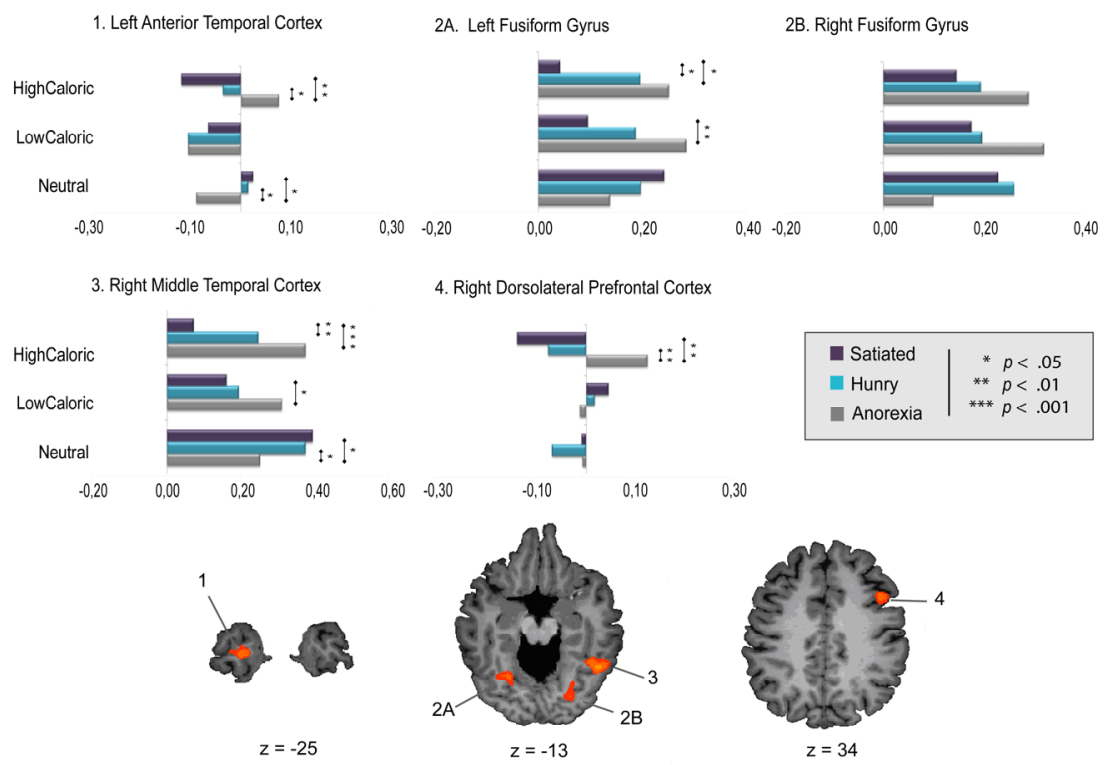

Figure 3.2 Results of the whole brain analyses of the stimulus $x$ group interaction for the bars attended conditions. Bar-plots represent mean BOLD signal change in z-scores.

Hypothesis 3: the neutral object conditions should not result in differences in neural activity between AN patients and healthy controls

For the neutral object colour attended condition, significant effects of group were found in the right OFC, the left and right AIC, the left dIPFC, the left VIPFC, the left premotor cortex, bilateral parietal cortex (precuneus), bilateral CACC, the left FEF and bilateral SMA. In contrast to what one would expect if these differences are the result of anemia, which decreases the BOLD response, pairwise comparisons showed that the AN patients had increased activity in these region compared to both hungry and satiated controls, with exception of the right OFC. It can therefore be concluded that the decrease in reward processing in AN patients, as discussed in the hypothesis 1 section, can therefore not be attributed to anemic effects. It might be suggested that this increased activity is the result of 
heightened attention, considering the functionality of the parietal cortex and the FEF. These regions are part of the fronto-parietal network and are involved in spatial attention possessing (Posner \& Petersen, 1990).

In the neutral object bars attended condition effects of group were found in the left aTempC and the right mTempC. Pairwise comparisons showed that the AN patients had decreased activity in the bars attended condition, compared to the hungry and satiated controls. However, if this were the result of anemia, one would expect a general decrease in all brain regions, which is not the case.

\section{DISCUSSION}

In the present study fMRI was used to investigate the hypothesized decrease in reward processing in AN patients, as compared to satiated and hungry control participants. The results show that the taste evaluation of high and low calorie foods increased activity in a network of brain regions, including those implicated in reward processing (i.e., the AIC and CACC) in the hungry and satiated healthy control participants, but not in the AN group. Apparently, food reward processing is reduced in $\mathrm{AN}$, even compared to satiated participants, when food stimuli are consciously evaluated.

Interestingly, when food cues were presented but participants did not explicitly attend to them, food reward processing (i.e., increased FG activity) in AN patients was observed as opposed to the healthy satiated but not hungry controls. This increased food reward processing was contiguous with increased activity in the right dIPFC and the left aTemp. Activity in the left aTemp has been previously related to the magnitude of the negative priming effect (Zubicaray, McMahon, Eastburn, Pringle \& Lorenz, 2006) and is thought to reflect the amount of effort to prevent responses to ignored objects. Increased activity in the right dIPFC has been previously implicated in the detection of conflict (Mansouri, Tanaka \& Buckley, 2009) and implementation of cognitive control (Hare, Camerer \& Rangel, 2009; Ridderinkhof, van den Wildenberg, Segalowitz \& Carter, 2004), Clearly, when AN patients are responsive to food temptations, they display an immediate compensatory increase in inhibitory self-control. But note that this was specific for the incidental exposure to the high calorie food pictures.

Finally, results of the neutral conditions indicate that these findings can not be 
attributed to the possible confounding effect of anemia in the AN patients, showing similar or even increased activity in the neutral condition for the AN patients compared to the healthy control participants. The increased activity may be the result of heightened attention in the AN patients, considering the functionality of the implicated regions, which included the parietal cortex and the FEF. These regions are part of the fronto-parietal network and are involved in spatial attention processing (Posner \& Petersen, 1990).

Both the AIC and ACC are important regions of the limbic circuitry, and have been previously implicated in the representation of interoceptive awareness of reward craving (Craig, 2009) and motivation of effortful reward-related behaviour (Walton et al., 2009) respectively. For example Nagvi et al. (2007) showed that smokers who acquire insula damage are very likely to quit smoking easily and to successfully remain abstinent. Walton et al. (2009) showed that a lesion of the ACC causes a bias away from a reward that requires effort to obtain. In line with these research findings, it seems that the impaired responsiveness of AIC and ACC during palatable food cue attendance are related to a decrease in food cravings and a decreased motivation to eat in AN patients. Indeed, the AN patients did show increased activity in the right anterior OFC in response to low calorie foods in the taste attended condition. A meta-analysis of neuroimaging studies (Kringelbach \& Rolls, 2004) indicates that the anterior OFC is involved in the processing of more abstract, secondary reinforcers such as money. This is in line with our findings in the unattended condition that food is still rewarding for AN patients. One might argue that low calorie foods are secondary reinforcers to particularly AN patients, as to them it signifies the maintenance of a desired low body weight.

Interestingly, present results show that the implicit processing of high calorie food stimuli results in both increased reward processing, but also cognitive control. A recent model proposed by Kaye and co-workers (Kaye, Fudge, \& Paulus, 2009), proposes that in AN patients a decrease in limbic processing is caused by exaggerated cognitive control by the dIPFC. Indeed, previous studies show that the dIPFC is implicated in successful self-control (Hare, Camerer, \& Rangel, 2009) and inhibition of eating behaviour in dieters (Delparigi et al., 2006). The present study however shows that increased activation of the right dIPFC and aTemp, was only found when food stimuli were not explicitly attended to. When food stimuli were not explicitly attended, their reward value was processed and 
cognitive control was increased. Clearly, the activated reward processing of highcalorie foods increased the need for control in AN. In case the reward system is not activated, by just viewing food pictures, there is no need for the cognitive control regions to be more active. It would be of interest to study the causality in future research. For example, what happens when the activity in relevant brain areas is modulated. by the use of transcranial direct current stimulation (Hecht, 2010; Tsai, 2005)? It might be hypothesized that experimental stimulation of the dIPFC during the unattended processing of food stimuli results in a decreased need to control and a corresponding increase in reward processing. 
Fighting food temptations: the modulating effects of cognitive reappraisal, suppression and up-regulation on mesocorticolimbic activity related to appetitive motivation 


\section{ABSTRACT}

Many popular diets claim that we can overcome the irresistible temptation of highly palatable foods, by actively controlling the way we think about foods. Testing this claim, participants in the present study were instructed to passively view foods, up-regulate food palatability thoughts, apply cognitive reappraisal (e.g., thinking about health consequences) or suppress food palatability thoughts and cravings. It was examined whether these strategies affected self-reported food craving and mesocorticolimbic activity as assessed by functional magnetic resonance imaging. Self-report results indicated that up-regulation increased food craving compared to other two conditions, but that there was no difference in craving between suppression and cognitive reappraisal. Corroborating the self-report results, the neuroimaging results showed that up-regulation increased activity in mesocorticolimbic regions. Contrary to our hypothesis, suppression more effectively decreased activity in the core of the mesocorticolimbic circuitry (i.e., ventral tegmental area and ventral striatum). However, results also show that suppression led to increased activity in the prefrontal cortex, suggesting that suppression required more self-regulatory effort compared to cognitive reappraisal. Overall, the results support the contention that appetitive motivation can be modulated by the application of cognitive control strategies. 
In most industrialized societies, where food is plenty, people often find themselves eating in the absence of any real hunger and all too often beyond direct energy requirements. This type of eating behaviour is thought to be the result of a strong appetitive motivation, which has been linked to activation of the mesocorticolimbic pathway (Alcaro, Huber, \& Panksepp, 2007; Kelley \& Berridge, 2002). This excessive appetite for particularly high calorie foods would undoubtly lead tow eight gain in the absence of some kind of control. Researchers have therefore proposed that eating behaviour is the outcome of an interplay between appetitive motivation and inhibitory cognitive control (Appelhans, 2009; Nederkoorn, Smulders, Havermans, Roefs, \& Jansen, 2006).

The suggestion that cognitive control can modulate mesocorticolimbic activity is in line with currently popular think slim diets, which propose that food cravings can be successfully decreased by actively controlling the way one thinks about foods (Beck., 2007; Stephens, 2007; Werrij, Jansen et al., 2009; Werrij, Roefs et al., 2009). If so, given that appetitive motivation is associated with mesocorticolimbic activity, one would expect that cognitive control would modulate this activity. There is already some research that has investigated the influence of such control on activity within the mesocorticolimbic circuitry. For example, previous neuroimaging studies show that word-level cognitive labels of odours and flavours can modulate activity in the orbitofrontal cortex (OFC; de Araujo, Rolls, Velazco, Margot, \& Cayeux, 2005; Grabenhorst, Rolls, \& Bilderbeck, 2008) and that that voluntary suppression of hunger feelings inhibits activity in mesocorticolimbic regions such as the amygdala, the hippocampus, the insula, the OFC, and striatum (Wang et al., 2009). In addition, in a previous study (Siep et al., 2009) we found increased mesocorticolimbic activity only when the task required participants to attend to the palatable taste, smell and texture of a presented visual food cue, but not when they were required to attend to a neutral aspect of the same food cue. These few studies suggest that it is possible that by the way one thinks of a food, or the manner in which one deliberately tries to perceive a given food, affects mesocorticolimbic activity. As mesocorticolimbic activity is related to appetitive motivation, this might influence how much food is eaten,

To further investigate the hypothesized interaction between appetitive motivation and cognitive control, the present study investigates the effects of three different types of cognitive control strategies known for their capability to modulate 
emotional responses (Gross, 2006); (1) up-regulation, which increases the intensity of innate responses, (2) cognitive reappraisal, which changes the way one thinks about emotion-eliciting cues in a way that changes its emotional impact, and (3) suppression, which is related to the active inhibition of thoughts (Wenzlaff \& Wegner, 2000) and emotional responses (Gross, 2006). Suppression is a form of emotion regulation that requires self-monitoring and self-corrective action throughout an emotional event. Such monitoring requires a high level of mental effort. In contrast, cognitive reappraisal is evoked early in the emotiongenerative process and does not require costly self-regulatory effort and can therefore be maintained for longer durations (Gross \& John, 2003).

Neuroimaging studies examining the neural bases of up-regulation, cognitive reappraisal and suppression, have found that each strategy differs in the way they influence brain activity. For example, up-regulating negative emotions increases medial prefrontal cortex (PFC) and decreases amygdala activity (Ochsner et al., 2004), while decreasing negative emotion by cognitive reappraisal increases activity in the lateral ventral and orbital PFC) and decreases activity in the amygdala, the ventral striatum and the insula (Ochsner et al., 2004; Wager, Davidson, Hughes, Lindquist, \& Ochsner, 2008). In addition, research indicates that suppression and cognitive reappraisal differ in their effects, showing that suppression increases PFC activity, but also increases amygdalar and insular responses (Goldin, McRae, Ramel, \& Gross, 2008), suggesting that cognitive reappraisal is somehow more effective in inhibiting mesocorticolimbic activity compared to suppression. Taken together, these results show that cognitive strategies are effective in modulating mesocorticolimbic activity involved in emotion processing, and suggest that they might also be effective in regulating mesocorticolimbic activity related to appetitive motivation.

In this study self-report measures and functional magnetic resonance imaging (fMRI) were used to investigate the influence of suppression, cognitive-reappraisal and up-regulation strategies on the subjective experience of food craving and mesocorticolimbic activity in healthy women. Participants were instructed to look at palatable food pictures and (1) imagine the food's palatable smell, texture and taste (i.e. up-regulation), (2) immediately inhibit any thoughts concerning food palatability and/or food cravings (i.e., suppression), or (3) focus on alternative meanings of the presented food cues, for example, the longer term consequences of consuming the food for their health (i.e., cognitive reappraisal). 
It was hypothesized that self-reported food cravings will be increased by the upregulation strategy, as compared to suppression and cognitive reappraisal. Furthermore, it was hypothesized that the cognitive reappraisal strategy would be more successful at decreasing self-reported food cravings, as compared to suppression. Concerning the neuroimaging data, it is expected that activity in mesocorticolimbic regions will be increased by up-regulation compared to both suppression and cognitive reappraisal, and that cognitive reappraisal will be more effective in decreasing mesocorticolimbic activity as compared to suppression. Lastly, in line with the theory by Gross and John (2003) it was hypothesized that suppression would require more self-regulatory effort as indicated by increased PFC activity as compared to cognitive reappraisal.

\section{METHOD}

\section{PARTICIPANTS}

Undergraduate students were recruited using flyers posted at Maastricht University. Given that women more often engage in nutritional self-assesment and dieting behaviour than men do (Davy, Benes, \& Driskell, 2006; Morse \& Driskell, 2009), only women were invited for an interview in which height, weight, age, handedness, medication use, (family) history of eating disorders and other psychiatric disorders, dietary restrictions, impulsivity, and reward responsiveness were assessed.

Candidates were excluded from participation when they disliked more than four of the foods used as stimuli in this study. Selected participants were 14 righthanded, non-dieting, healthy students with a healthy body weight (body mass index (BMI) between 18.5 and $25[M=21.5, S D=1.9]$ ), a score of $<15$ on the Restraint Scale $[M=10.7, S D=1.9$; Herman, Polivy, Pliner, Threlkeld, \& Munic, $1978]$, and with a mean age of $21.1(S D=1.5)$. Because food intake varies across the menstrual cycle in females (Bryant, Truesdale, \& Dye, 2006), participants were selected based on the use of combined oral contraceptives (COCs). COCs inhibit the production of fertility hormones and consequently prevent increases in food intake in the premenstrual phase (Goldzieher, 1994). Further, because the personality characteristics of impulsivity and reward responsiveness are supposed to reflect the sensitivity of the reward system (Davis et al., 2007) participants 
were screened on reward responsiveness and impulsivity. All participants scored within the normative population range of impulsivity as measured with the Barratt Impulsiveness Scale [Spinella, 2007; Impulsivity score participants: $M=59.7, S D=4.9$; normative score: $M=64.2, S D=10.7]$. In addition, participants scored within the normative population range of reward responsiveness as measured with a subscale from the BIS/BAS scale [Carver \& White, 1994; participants' score: $M=16.7, S D=1.5$; normative score: $M=17.5$, $S D=1.4]$.

\section{STIMULI}

In a pilot study, 25 savoury and 25 sweet high calorie food pictures were selected as candidate stimuli from an internet database (www.istockphoto.com). Subsequently, these food pictures were rated on $100 \mathrm{~mm}$ visual analogue scales (VAS) by 20 healthy, non-dieting female volunteers not participating in the actual study on calorie content (0: very low calorie - 100: very high calorie) and palatability (0: very bad tasting - 100: very good tasting). Based on these ratings, 14 sweet, palatable, high calorie food pictures [calorie content ratings: $M=82.1$, $S D=4.8$; palatability ratings: $M=65.7, S D=6.9$; e.g., chocolate, cake, cookies, ice cream] and 14 savoury, palatable, high calorie food pictures [calorie content ratings: $M=80.7, S D=4.6$; palatability ratings: $M=67.2, S D=4.6$; e.g., pizza, fries, crisps, hamburger] were selected. The selected food pictures were projected as pop-out figures on a black canvas to minimize noise input (Rainer, Augath, Trinath, \& Logothetis, 2001). For a more elaborate discussion on pop-out figures and the use of pictures instead of real foods, we refer to a previous fMRI study (Siep et al., 2009). Only pictures of high calorie foods were included, as it is expected that healthy, lean women will normally only apply cognitive control strategies to high calorie foods and not to low calorie foods.

\section{DESIGN AND EXPERIMENTAL TASK}

This study used a one-way within-subjects design with four levels (cognitive control strategy: cognitive reappraisal, suppression, up-regulation, and passive viewing). To avoid carry-over effects from the cognitive control conditions to the passive viewing condition, all participants started the experiment with a passive viewing task. The results of this task were used as a loose comparison control 
condition for post-hoc interpretations. In the passive viewing task, pictures of food were pseudo-randomly presented in the centre of the screen. To ensure that the participant maintained a constant level of attention, she was instructed to press a button with her right index finger as soon as a dot appeared in the centre of a food picture. Dots appeared in $13 \%$ of the food picture trials and these trials were later removed from analyses.

The passive viewing task was followed by a practice task to familiarize the participant with the cognitive control conditions and the symbols representing the conditions: eye, stop sign, and upward arrow (see Figure 4.1 for an overview). Training of the participant included initial instructions, followed by practice as the investigator observed and shaped her technique. The participant was specifically instructed not to look away from the images or to close her eyes. The eye symbol indicated that the participant should think about the negative consequences of eating the presented food for her weight, health, and bodily appearance (i.e. cognitive reappraisal). This type of reappraisal has also been referred to as situation-focussed reappraisal (Ochsner et al., 2004), in which the participant is to reinterpret the actions and outcomes for a given image. The stop sign indicated that the participant should look at the presented food cue in a neutral way and immediately inhibit any thoughts (Wenzlaff \& Wegner, 2000) or responses (Gross, 2006) related to the palatability of the presented food, without looking away from the picture (i.e., suppression). The upward arrow indicated that the participant should increase their cravings for the presented food cues by actively thinking about the delicious smell, taste, and texture of the presented food cue in a way that would make her mouth water (i.e., up-regulation).

Once the participant had mastered the technique to the satisfaction of the investigator, two experimental runs followed in which the participant applied the cognitive control strategies. In these two runs, the participant was first shown the symbol, cueing which type of cognitive control strategy she should apply. To make the application of the three cognitive control strategies less difficult and confusing, two food pictures were presented, during which the participant applied the cued cognitive strategy twice in a row. The presentation of the two food pictures was followed by a vertical $100 \mathrm{~mm}$ VAS, assessing experienced craving (item: "I have cravings for one or more specific foods", top: I totally agree (100) - bottom: I totally disagree (0)). This item was taken from the Food Craving Questionnaire (Moreno, Rodriguez, Fernandez, Tamez, \& Cepeda-Benito, 2008). 


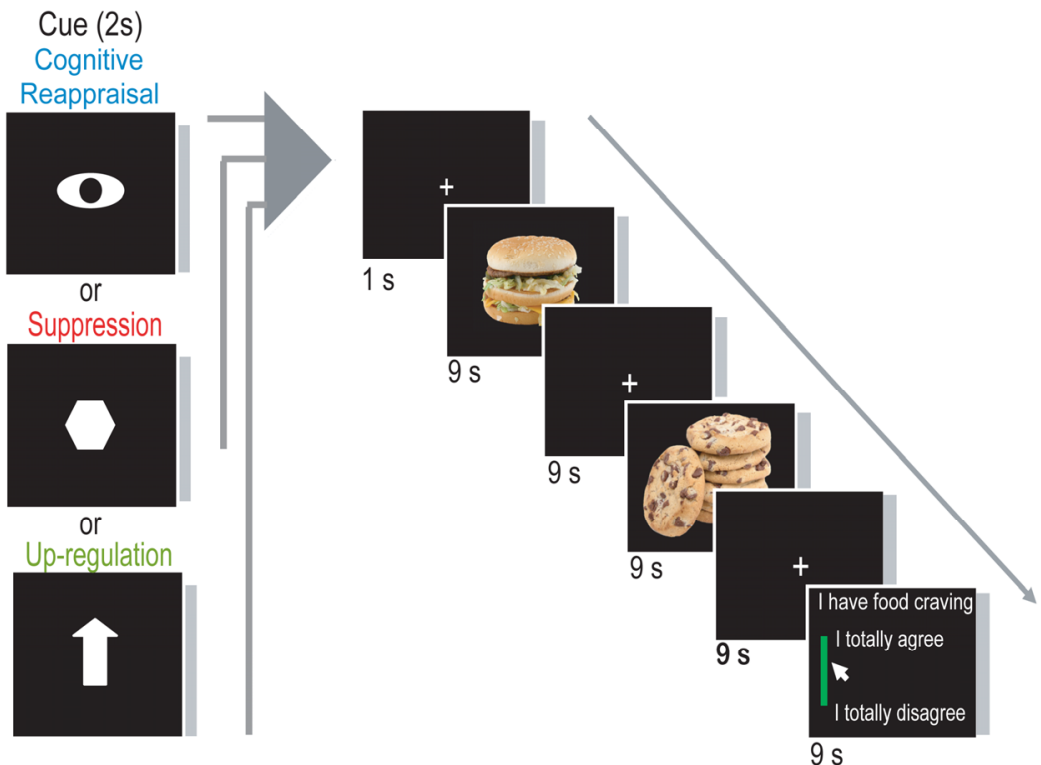

Figure 4.1 Graphical outline of the stimulation protocol. Stimuli were presented in a slow-eventrelated design and involved three cognitive control conditions: up-regulation, cognitive reappraisal and suppression. During the tasks participants focused on the screen centre as indicated by the white fixation cross. Stimuli were presented in the centre of the participant's visual field. At the beginning of each block a cue was presented indicating the task.

\section{STIMULATION PROTOCOL}

The experiment consisted of a total of three experimental runs in which food pictures were presented in a slow event-related fashion, and one anatomical scan. During the passive viewing run, the presentation of each food picture trial lasted $9 \mathrm{~s}$, followed by a white fixation cross $(9 \mathrm{~s})$. In 4 of the food picture trials, a white dot appeared for $200 \mathrm{~ms}$, after a variable time length from the first presentation of the food picture. The participant was instructed that fixation should be maintained throughout the passive viewing run. In total, the passive viewing run lasted approximately 9 mins. For each participant, the order of the 14 savoury and 14 sweet food pictures within the passive viewing run was randomized, and comprised 32 trials including the 4 "dot trials". Each picture was presented once per condition.

In the two cognitive control runs, each cognitive control strategy was presented 7 
times in a pseudo-random order. During each type of cognitive control condition (see Figure 4.1), an initial cue appeared ( $2 \mathrm{~s}$ ), indicating which cognitive control strategy the participant should apply, followed by a white fixation cross ( $1 \mathrm{~s}$ ). Then two food picture trials were presented sequentially, lasting $9 \mathrm{~s}$ each, followed by fixation ( $9 \mathrm{~s}$ ). Presentation of the two food pictures was followed by a VAS $(9$ s). Although the duration of the VAS trial remained constant, the VAS disappeared as soon as the participant made her choice. Throughout one cognitive control run, each condition was repeated seven times. Each cognitive control run lasted approximately 20 mins.

The order of the three cognitive control conditions was randomized per run and then manually checked for repetitions to avoid fMRI adaptation effects (GrillSpector \& Malach, 2001). For each participant, the order of food stimuli per condition within each run was fully randomized. The order of the two cognitive control runs was balanced across participants. The total fMRI session lasted 75 mins.

\section{PROCEDURE}

All imaging sessions took place around lunch-time (i.e., between 1 and $3 \mathrm{pm}$ ). Participant were instructed not to consume any food or beverages (except water) 4 hrs prior to the imaging session. Upon arrival, written informed consent and ethical approval were obtained from the participant. Next, hunger was assessed with 100 mm VAS (Friedman, Ulrich, \& Mattes, 1999, translated into Dutch), which showed that participants were moderately hungry at the start of the fMRI experiment $[M=48.7, S D=19.5]$. After completing the questionnaire, the participant entered the scanner. The participant did not receive any instructions about the different experimental tasks, to avoid influences from the cognitive control instructions on the passive viewing task. After completion of the passive viewing task, an anatomical scan was performed during which the participant received the instructions for the control tasks. The practice task was followed by the two experimental runs in which the participant applied the practiced cognitive control conditions. At the end of the fMRI session, the participant completed an exit questionnaire inquiring about the methods she applied during the cognitive control runs and the experienced difficulties of the cognitive control conditions. After completing the study, the participant received $€ 15$ compensation. 


\section{fMRI DATA ACQUISITION}

Images were acquired with a 3T Siemens Magnetom Allegra Head-only Scanner at the Maastricht Brain Imaging Centre (MBIC) using a birdcage volume coil. Gradient-echo planar imaging (EPI) volumes were acquired (50 slices, $\mathrm{TR}=3000 \mathrm{~ms}$ ). Imaging parameters were optimized to minimize susceptibility and distortion artefacts in the OFC (see: Weiskopf, Hutton, Josephs, \& Deichmann, 2006). The relevant factors included oblique axial imaging with a negative (i.e. backward) tilt angle of 30 degrees, minimizing voxel size $(2 \times 2 \times$ $2.5 \mathrm{~mm}$ ) in the plane of imaging, a short echo time of $25 \mathrm{~ms}$, and a high imaging bandwidth $(2790 \mathrm{~Hz}$ over the field of view, echo spacing $=0.4 \mathrm{~ms})$. The voxel matrix size was $128 \times 104 \mathrm{~mm}$, and the field of view (FoV) was $256 \times 208 \mathrm{~mm}$. Acquisition of functional images yielded 200 volumes during the passive run and 409 during each cognitive modulation run. One high-resolution whole-brain anatomical T1-weighted scan was acquired: an optimized MPRAGE sequence $(\mathrm{TR}=2250 \mathrm{~ms}, \mathrm{TE}=2.6 \mathrm{~ms}$, flip angle $=9$ degrees, $1 \times 1 \times 1 \mathrm{~mm})$.

\section{fMRI DATA PREPROCESSING}

All processing and analyses of the fMRI data were performed using Brainvoyager QX version 1.9 (Brain Innovations, Maastricht, The Netherlands). The first two volumes of the $\mathrm{T}^{*}$ weighted functional images were discarded due to magnetic saturation effects. Preprocessing comprised slice scan timing correction (using sinc interpolation), motion correction (using a 3D rigid-body transformation of each volume to the first volume of each run and using sinc interpolation) and high-pass filtering to remove low-frequency noise (up to 3 cycles in the single run time-course). Individual functional data were smoothed using a $6 \mathrm{~mm}$ full-widthat-half-maximum isotropic Gaussian Kernel. The anatomical scan and the functional data were then spatially normalized using Talairach transformation procedures (Talairach \& Tournoux, 1988). For group analysis, the normalized individual functional data were averaged, accounting for both scan-to-scan and participant-to-participant variability.

\section{fMRI ANALYSIS}

A whole-brain, voxel-wise Random Effects (RFX) ANOVA was used to test for differences in BOLD signal across cognitive control strategies. Because our 
hypothesis concerned the modulation of mesocorticolimbic activity by the three modulation conditions, only the suppression, cognitive reappraisal, and upregulation conditions were included at this stage of the analysis. The results of the passive viewing condition were included for post-hoc interpretations.

The resulting $F$-map of the RFX ANOVA, F-test main effect of cognitive control strategy, was thresholded at a significance level of $p<.01$. To correct for multiple comparisons, a minimum cluster size of 9 contiguous voxels was adopted (determined by a clustersize treshold estimator plugin implemented in BrainVoyager), yielding a whole-brain corrected statistical threshold of $\alpha<.05$.

To test our three hypotheses, we used the functional region of interest (fROI) approach, which involves identifying a priori hypothesized candidate regions in the PFC and mesocorticolimbic, from the corrected whole-brain F-map and then calculating the overall summary measure of response in each region (see: Saxe, Brett, \& Kanwisher, 2006). This method allowed us to decrease the influence of noise that varies between voxels and make inferences about the response profile of each $\mathrm{fROI}$ as a whole, rather than particular voxels within the region.

Our corrected F-map comprised a total of 24 fROls (Table 4.1), of which 11 fROls were located in the a priori hypothesized PFC and mesocorticolimbic circuitry. The beta weights for each condition, including passive viewing, were averaged across participants, and across all voxels within each of these 11 fROls. Bar-plots were created to visualize the response profiles of all fROIs (Figure 4.2). Following our hypothesis, fROls were divided into two groups: regions located in mesocorticolimbic circuitry but outside the PFC (Figure 4.2A), and regions located in the PFC (Figure 4.2A). A functional region of $\mathrm{fROI}$ analysis was performed to evaluate differences in the magnitude of the MR signal change between conditions, using pairwise $t$-tests. $T$-tests were considered statistically significant at $\alpha=.01$ and planned comparisons at $\alpha=.05$. Results of these $t$-tests are depicted in (Figure 4.2), and were the focus of the discussion. 
Table 4.1 Results whole brain RFX ANOVA, F-test: main effect of cognitive control strategy.

\begin{tabular}{lccrrc}
\hline & & Talairach coordinates & & & \\
Functional Region of Interest & $\mathrm{L} / \mathrm{R}$ & $(\mathrm{x}, \mathrm{y}, \mathrm{z})$ & $\mathrm{BA}$ & $\mathrm{F}$-score & $p$ - value \\
\hline Anterior Temporal Cortex & $\mathrm{L}$ & $-43,5,-30$ & 21 & 8.48 & $<.001$ \\
Cerebellum & $\mathrm{L}$ & $-31,-81,-27$ & - & 9.32 & $<.001$ \\
Medial Orbitofrontal Cortex & $\mathrm{L}$ & $-12,19,-7$ & 11 & 3.33 & .03 \\
Inferior Temporal Gyrus & $\mathrm{R}$ & $58,-47,-11$ & 20 & 6.28 & .001 \\
Ventral Tegmental Area & $\mathrm{L}$ & $-5,-14,-7$ & - & 6.22 & .002 \\
Fusiform Gyrus & $\mathrm{L}$ & $-29,-56,-15$ & 37 & 9.51 & $<.001$ \\
Anterior Prefrontal Cortex & $\mathrm{R}$ & $23,65,11$ & 10 & 10.06 & $<.001$ \\
& $\mathrm{~L}$ & $-22,61,17$ & 10 & 8.49 & $<.001$ \\
Ventromedial Prefrontal Cortex & $\mathrm{L}$ & $-9,24,-1$ & 24 & 4.74 & .007 \\
Lateral Orbitofrontal Cortex & $\mathrm{L}$ & $-46,49,4$ & 11 & 10.71 & $<.001$ \\
Ventral Striatum & $\mathrm{L}$ & $-11,-2,-3$ & - & 6.22 & .002 \\
Parastriatal Cortex & $\mathrm{R}$ & $22,-94,12$ & 18 & 9.50 & $<.001$ \\
& $\mathrm{~L}$ & $-14,-95,14$ & 18 & 7.02 & $<.001$ \\
Operculum & $\mathrm{L}$ & $-58,-19,26$ & 41 & 10.94 & $<.001$ \\
Posterior short Insular gyrus & $\mathrm{R}$ & $73,-8,11$ & 13 & 10.71 & $<.001$ \\
& $\mathrm{~L}$ & $-38,-6,11$ & 13 & 12.33 & $<.001$ \\
Posterior Cingulate Cortex & $\mathrm{L}$ & $-9,-53,8$ & 30 & 8.65 & $<.001$ \\
Dorsolateral Prefrontal Cortex & $\mathrm{R}$ & $41,53,33$ & 9 & 10.71 & $<.001$ \\
Somatosensory Cortex & $\mathrm{R}$ & $-55,-11,31$ & 3 & 11.14 & $<.001$ \\
& $\mathrm{~L}$ & $-56,-4,34$ & 3 & 10.94 & $<.001$ \\
Inferior Parietal Cortex & $\mathrm{R}$ & $41,-47,43$ & 40 & 4.66 & .007 \\
Frontal Eye Field & $\mathrm{R}$ & $28,-10,55$ & 6 & 3.64 & .02 \\
Extrastriate cortex & $\mathrm{R}$ & $17,-82,40$ & 19 & 5.08 & .004 \\
\hline
\end{tabular}

Note: $\mathrm{L}=\mathrm{Left}, \mathrm{R}=$ Right, $\mathrm{BA}=$ Brodmann area; $\mathrm{fROIs}$ in Italic.

\section{RESULTS}

\section{MANIPULATION CHECK}

To test whether the participants followed the instructions, they were asked to describe their applied cognitive control strategies in an exit questionnaire. From these reports, we concluded that all participants did indeed follow our instructions as intended as their description matched the practise task instructions. The exit questionnaire also included two $100 \mathrm{~mm}$ VAS, in which participants rated their experienced difficulties in applying each of the three modulation tasks [0: very difficult - 100: very easy]. Results showed that there was a main effect of cognitive modulation on the experienced difficulty $[F(2,26)$ $=5.10, p=.03]$. Pairwise comparisons showed that there was a significant increased in self-reported difficulty for the cognitive reappraisal task compared to up-regulation $[p=.02$; up-regulation: $M=16.9, S D=6.8$; cognitive reappraisal: 
$M=45.9, S D=7.0]$ and suppression was also considered to be easier than cognitive reappraisal $[p=.05$; suppression: $M=30.4, S D=6.6]$. Furthermore, up-regulation did not differ in experienced difficulty from suppression $(p=.59)$.

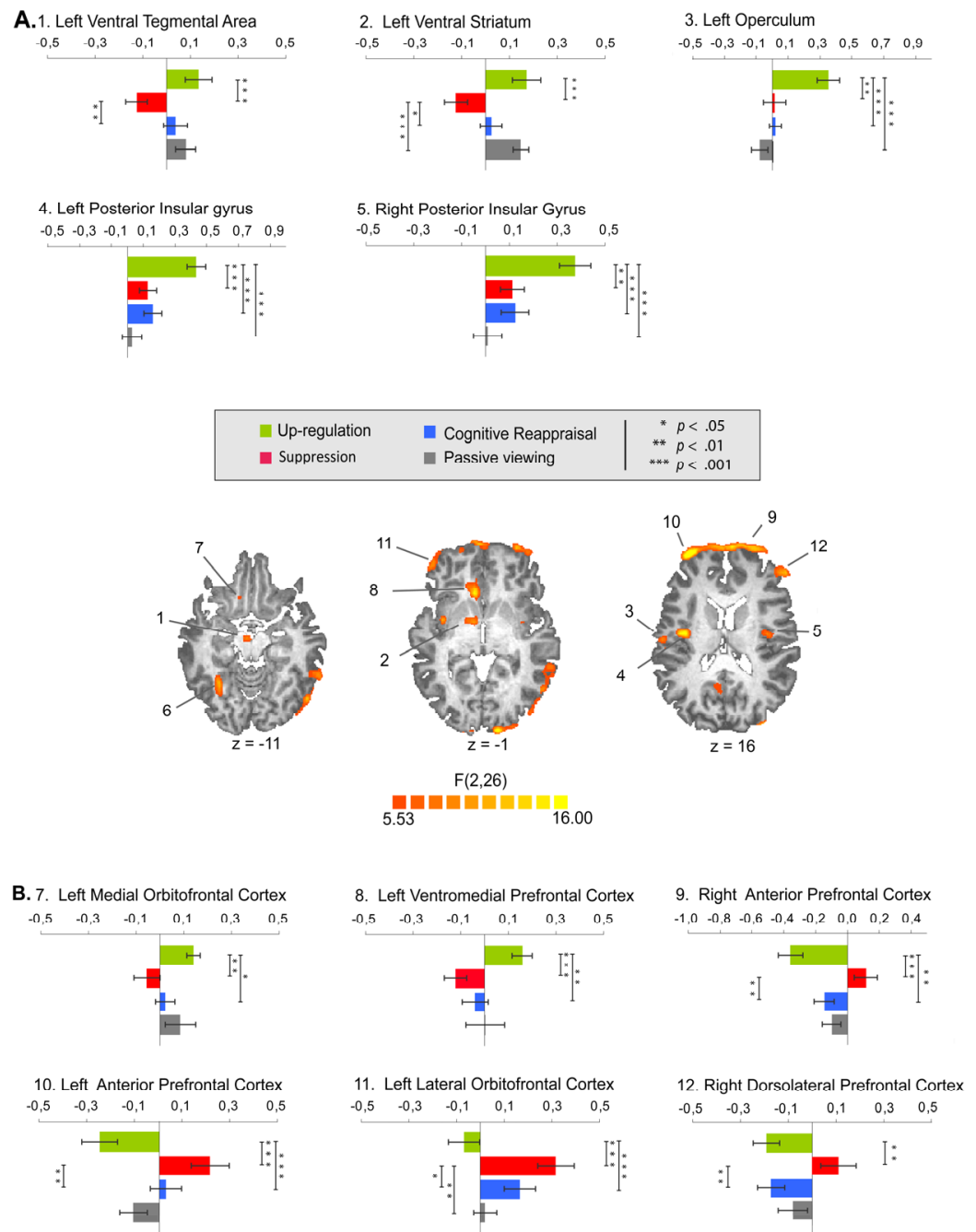

Figure 4.2 Results RFX ANOVA, F-test: cognitive control strategy, $p<.01$. fROI bar-plots represent BOLD signal change in z-scores, \pm SEM. A: Results for fROIs located outside the PFC. B: Results for fROls located in the PFC. 


\section{SELF-REPORTED FOOD CRAVING}

There was a significant main effect of cognitive control strategy on experienced food craving $[F(2,26)=43.37, p<.001$; cognitive reappraisal: $M=50.1, S D=$ 18.0; suppression: $M=52.1, S D=18.8$; up-regulation: $M=70.3, S D=18.3$ ]. Pairwise comparisons showed that subjective food craving after the up-regulation trials was significantly increased as compared to that following the cognitive reappraisal $(p<.001)$ and suppression $(p<.001)$ trials, which is in line with out hypothesis. In contrast to what was expected however, subjective ratings of craving did not differ between the cognitive reappraisal trials and suppression trials $(p=.83)$.

\section{fMRI RESULTS}

The whole brain analysis of the effect of cognitive control strategy (cognitive reappraisal, suppression vs. up-regulation) on blood oxygenation level-dependent (BOLD) activity revealed a network of fROls showing significant effects (Table 4.1). The resulting F-map included six fROIs located within the mesocorticolimbic system, including the left ventral tegmental area (VTA; Duzel et al., 2009), the left ventral striatum (VS; Kreitzer, 2009), the left operculum (Schweinhardt, Seminowicz, Jaeger, Duncan, \& Bushnell, 2009) and bilateral posterior insular gyrus (PIG; Craig, 2009; Figure 4.2A); and six fROIs located in the PFC, including the left medial orbitofrontal cortex (OFC), the left ventromedial prefrontal cortex ( $\mathrm{vmPFC})$, bilateral anterior prefrontal cortex (aPFC), the left lateral orbitofrontal cortex (IOFC) and the right dorsolateral prefrontal cortex (dIPFC; Figure 4.2B).

In line with the first hypothesis of increased mesocorticolimbic activity during upregulation compared to both suppression and cognitive reappraisal, results showed that up-regulation increased activity as compared to suppression in all six mesocorticolimbic fROls located outside the PFC [VTA: $t(13)=5.88, p<$ .001 ; VS: $t(13)=5.47, p<.001$; operculum: $t(13)=3.39, p<0.01$; left PIG: $t(13)$ $=5.30, p<0.001$; the right PIG: $t(13)=4.03, p<0.01]$ and two regions located in the PFC [mOFC: $t(13)=2.50, p<.05$; vmPFC: $t(13)=2.50, p<.05$ ]. Upregulation showed increased activity as compared to cognitive reappraisal in the left operculum $[t(13)=5.55, p<.001]$, the left PIG $[t(13)=5.13, p<.001]$, the right PIG $[t(13)=4.48, p<.001]$. However, up-regulation did not significantly 
differ from cognitive reappraisal in the VTA $[t(13)=0.66, p=.52]$ and the left VS $[t(13)=0.59, p=.67]$ as suppression did. Up-regulation did significantly increase activity as compared to cognitive reappraisal in the mOFC $[t(13)=2.50, p<.05]$ and vmPFC $[t(13)=2.50, p<.05]$.

Surprisingly, in contrast to the second hypothesis of more successful inhibition of mesocorticolimbic activity during cognitive-reappraisal compared to suppression, results showed that suppression significantly inhibited activity the left VTA [ $t(13)$ $=3.45, p<.01]$ and the left ventral striatum $[t(13)=2.77, p<.05]$ as compared to cognitive reappraisal. These findings suggest that suppression is more successful at inhibiting mesocorticolimbic activity as compared to cognitive reappraisal.

In line with the third hypothesis of increased PFC activity during suppression compared to cognitive reappraisal, results showed that suppression significantly increased activity in the right anterior PFC [aPFC; Figure 4.2B; $t(13)=3.43, p<$ $.01]$, the left aPFC $[t(13)=3.07, p<.01]$, the left lateral OFC $[$ IOFC; $t(13)=2.79$, $p<.05]$, and the right dorsolateral PFC [dIPFC; $t(13)=4.49, p<.01$ ] compared to cognitive reappraisal.

\section{DISCUSSION}

In this study self-report measures and fMRI were used to examine the regulatory effects of up-regulation, cognitive reappraisal, and suppression on appetitive motivation. Self-report results indicated that up-regulation increased food craving, and that this increase was accompanied by increased activity in mesocorticolimbic regions, including the VTA, the VS, the Operculum, the PIG, the mOFC and the vmPFC. Self-reports did not show differences the between cognitive reappraisal and suppression task, but the neuroimaging results did. Surprisingly, suppression decreased activity in the VTA and VS more successfully than cognitive reappraisal, suggesting that suppression is more effective in inhibiting mesocorticolimbic activity. However, results also indicate that suppression increases activity in the bilateral aPFC, the IOFC and dIPFC as compared to cognitive reappraisal, which is in line with the proposition that suppression requires increased self-regulatory effort (Gross \& John, 2003). Interestingly this effect cannot be ascribed to a greater complexity of the 
suppression task, as participants indicated that they found the cognitive reappraisal task to be more difficult. Nonetheless, it is conceivable that the rated difficulty of the cognitive reappraisal task rather reflects the participants' degree of relative unfamiliarity with this strategy.

The present findings show that women can actively modulate mesocorticolimbic activity related to appetitive motivation by the application of up-regulation, suppression and cognitive reappraisal, and that the specific effects depend on the type of cognitive control strategy applied. Although suppressions seems more effective at inhibiting corticomesolimbic activity compared to cognitive reappraisal, results also indicate that suppression requires more self-regulatory effort as indicated by the increase in PFC activity.

In contrast to present findings, previous research (Wang et al., 2009) indicated that women who are instructed to inhibit hunger feelings fail to inhibit activity in the VS, insula and OFC compared to men. From these findings authors conclude that women have lower abilities to suppress hunger feelings compared to men. Present results however show that women can successfully suppress activity within the insula, OFC and VS. Differences in results might be explained by the differences in instruction. Furthermore, in the study by Wang et al. participants received a general instruction to decrease their feelings of hunger, which might have caused differences between men and women in the type of strategy applied. Present findings indicate that if the women more frequently applied cognitive reappraisal, this indeed does not result in decreased activity in the VS. However, this does not necessarily imply that they are less successful. Future research is necessary to test the hypothesized difference in type of cognitive control strategy applied between men and women.

The finding that healthy women can effectively regulate mesocorticolimbic activity may be relevant for treatments of obesity. There is now considerable support that obese people show abnormalities in the mesocorticolimbic circuitry, which may cause them to overeat (Rothemund et al., 2007; Stice, Spoor, Bohon, \& Small, 2008; Stoeckel et al., 2008; Volkow \& Wise, 2005; Wang et al., 2001). One could hypothesize that the treatment of obesity should target and restore these abnormalities. Results of drug treatments targeting mesocorticolimbic food reward neurotransmission (Astrup et al., 2007; Hu, Zhu, \& Huang, 2009) show that they are indeed effective at reducing weight. However, due to their non-specificity, they seem to affect reward processing in 
general, which increases the risk of adverse psychiatric events (e.g., depression, anxiety, and suicidal ideation). Therefore, it is necessary to find alternative methods to target abnormalities in mesocorticolimbic processing of obese people. One such method may be some form of cognitive behavioural therapy with a focus on training cognitive control strategies such as cognitive reappraisal of palatable foods and suppression of strong food cravings.

In conclusion, the present study indicates that healthy women can modulate activity within the mesocorticolimbic circuitry using cognitive control strategies. This finding is in support with previous studies indicating that eating behaviour can be successfully inhibited by actively controlling the way one thinks about foods (Beck., 2007; Stahre \& Hallstrom, 2005; Werrij, Jansen et al., 2009). Present results provide additional insight into neurobiological mechanisms underlying the cognitive control of appetitive motivation, which might aid in development of effective abnormal eating behaviour treatment. For example, considering the observed hypoactivity in the PFC of obese individuals (Volkow et al., 2008), one might suppose that they are less effective in suppressing reward processing. Therefore, cognitive reappraisal might be a more effective strategy. However, present findings also indicate that suppression is more effective at directly inhibiting activity in the VTA and VS, the core of the mesocorticolimbic circuitry. Therefore, it can also be hypothesized that training self-regulatory abilities, for example by training working memory (Hofmann, Gschwendner, Friese, Wiers, \& Schmitt, 2008) might increase the ability to effectively suppress appetitive motivation and restrict of food intake. It is suggested that future research should focus on the application of cognitive control strategies as a method for targeting abnormal mesocorticolimbic activity in overweight people and patients with eating disorders. 


\section{Cognitions and emotions in eating disorders}


Imagine that you are sleeping in your bedroom. In the middle of the night you suddenly awake of a loud noise downstairs in the living room. You think 'o no, there is a burglar in my house'. You feel extremely anxious. You do not dare to go downstairs to have a look in the living room; that is why you stay where you are, trembling and waiting for what is coming. Now imagine the following situation. You are sleeping again. In the middle of the night you suddenly awake of a loud noise downstairs in the living room. You think 'that stupid cat! What did she knock over this time?' You feel a bit irritated but soon continue your sweet dreams. These examples make clear that it is not what is really happening that causes one to feel anxious, irritated or happy. It is how one interprets what is happening that determines one's feelings. In EDs (EDs) it is not one's actual appearance or body weight that causes a problem, but one's evaluation of it. This is a key assumption of the cognitive model of EDs.

\section{COGNITIVE BIASES}

The cognitive model of EDs has its roots in the cognitive model of psychopathology or mental disorders that was formulated by Beck in 1964. Beck theorized that the processing of external events or internal stimuli is biased in mental disorders. These biases, or cognitive errors, systematically distort the individual's experiences. The model points out that eating psychopathology arises from maladaptive knowledge structures (e.g., schemas) that are involved in the allocation of attention, in memory, and in the interpretation of incoming information (Hargreaves \& Tiggemann, 2002; Williamson, White, York-Crowe, \& Stewart, 2004). Activation of these knowledge structures causes disorder-relevant information to be processed in a biased manner, resulting in a range of cognitive biases in attention, judgment and memory (Williamson et al., 2004).

In line with Beck's (1976) cognitive specificity hypothesis, Vitousek and Hollon (1990) proposed two decades ago that ED patients consider their own weight and shape as the predominant referents for inferring personal value. Nowadays this is also referred to as 'overevaluation' (Fairburn, 2008). These cognitions about weight and shape in relationship to the self are organized into structures referred to as weight-related self-schemata. The central premise of the cognitive model is that these schemata are the core cognitive component of EDs. The operation of 
the schemata might cause and/or maintain EDs by producing systematic errors in weight and shape information processing.

The cognitive model specifies three main cognitive errors in EDs: attention bias, interpretation bias and memory bias, and some recent studies suggest impairments in general cognitive processing. In this chapter we will first discuss the main cognitive biases and general impairments in cognitive processing that are demonstrated in EDs. We then will focus on the question whether the manipulation of cognitive processing affects ED psychopathology.

\section{ATTENTION BIAS}

An attention bias refers to the tendency to selectively attend to disorder-relevant stimuli (e.g., Mathews \& MacLeod, 2005). According to the cognitive model of EDs, ED patients are more likely to give priority to cues pertaining to body and food-related information than to neutral cues, in comparison to healthy controls. The cognitive model proposes that, with progression of the ED, a phobic orientation toward the body, high calorie foods, and weight gain develops (Williamson, Muller, Reas \& Thaw, 1999). The hyper-attention to disorder relevant cues is presumed to maintain EDs: it might lead to the avoidance of cues that elicit anxiety and negative affect. This avoidance will immediately decrease anxiety but it will also prevent its extinction. Therefore, in the long run, anxiety and negative affect will maintain or even increase and the ED will continue. Several paradigms have been used to test the attention bias hypothesis in EDs, including the modified Stroop task, the dot-probe task, the visual search task and eye tracking.

\section{STROOP TASK}

The Stroop task is the most frequently used paradigm to investigate attention bias in ED patients. Neutral words and ED relevant words are printed in different colors (Faunce \& Job, 2000; Williams, Mathews \& MacLeod, 1996). Participants are required to ignore the meaning of the words and to simply report the color in which each word is printed. Disorder relevant words interfere with color naming 
more than neutral words do, leading to longer color-naming responses for disorder relevant words than for neutral words. This difference in response time for disorder relevant versus neutral words is the 'interference effect'. Currently, more than 30 studies have evaluated the interference effect for food-, weight- and shape-related words in ED patients. Confirming the attention bias hypothesis, it was concluded in a meta-analysis that ED patients show increased interference for food-, weight- and shape-related words (Johansson, Ghaderi, \& Andersson, 2005). Both patients with bulimia nervosa (BN) and anorexia nervosa (AN) showed the Stroop interference for weight- and shape-related words, but AN patients were more interfered by food stimuli relative to BN patients (but see Dobson \& Dozois, 2004).

Although a large number of studies used the modified Stroop task to demonstrate attention biases in EDs, a number of concerns regarding the use of this task have been raised. In a review of modified Stroop studies in EDs, Lee and Shafran (2004) conclude that Stroop interference with ED-related stimuli is also found in non-ED groups, which brings into question its clinical relevance. More specifically, these biases have been demonstrated in restrained eaters (Francis, Stewart, \& Hounsell, 1997), hungry participants (Mogg, Bradley, Hare \& Lee, 1998), food deprived participants (Placanica, Faunce, \& Soames Job, 2002), and in healthy participants who had just finished an appetizer (Overduin, Jansen, \& Louwerse, 1995). Another, more general, concern about the Stroop task is that little effort has been made to account for the underlying mechanisms of the interference effect (Williams et al., 1996). It is not clear why ED patients show interference. Food, weight and shape words might trigger anxiety in ED patients, who usually experience strong concerns pertaining to their body weight, shape and eating, hence making these words especially meaningful and frightening. The anxiety might induce an automatic tendency to avoid further exposure to these words. Interference then might reflect avoidance and not any attention bias. Indeed, De Ruiter and Brosschot (1994) demonstrated that attempts to cognitively avoid the processing of disorder-relevant word stimuli also results in increased interference scores.

Thus, the central premise that attention is biased toward food and body stimuli cannot be tested with the modified Stroop task, because it is not clear what the Stroop task precisely measures. Given this uncertainty about the meaning of increased interference scores, no firm conclusions can be drawn about the 
existence of an attention bias in ED patients using the modified Stroop paradigm. Therefore, alternatives to the Stroop task were used, such as the dot-probe task.

\section{DOT-PROBE TASK}

The dot-probe task (MacLeod, Matthew \& Tata, 1986) is based on the assumption that individuals respond faster to a small dot that is presented in an attended area of a visual display than to a dot that is presented in an unattended area of a visual display. Pairs of pictures or words are concurrently and briefly presented to participants. One word/picture of each pair is disorder relevant and the other is neutral. When the word/picture pair disappears, a small dot appears in a position previously occupied by one of the two words or pictures. The participant is instructed to push a button as quickly as possible when the dot appears. The attention bias for disorder relevant stimuli is calculated by taking the difference in reaction times to the dot when it replaces the neutral stimulus minus reaction times to the dot when it replaces the disorder relevant one. Faster reactions to dots that replace the disorder relevant stimuli indicate an attention bias.

The first study using the dot-probe task in EDs (Rieger et al.,1998) tested the attention bias for shape-related words. Results showed that the ED group detected a target dot that replaced a thin shape-related word slower than did healthy individuals. In contrast, the ED group detected a target dot that replaced a large shape-related word faster than did healthy individuals. Rieger and colleagues concluded that ED patients are more likely to attend to information consistent with fatness and to ignore information consistent with thinness, which might maintain distorted cognitions about shape and weight.

Rieger et al. (1998) used words but Mogg and colleagues argue that using words as stimuli provides a relatively fragile index of attention bias, and they suggest using pictures instead (Mogg, Bradley, Dixon, Fisher, Twelftree \& McWilliams, 2000). Pictorial dot-probe studies were also done to study ED attention biases (Glauert, Rhodes, Fink \& Grammer, 2010; Lee \& Shafran, 2008; Shafran, Lee, Cooper, Palmer \& Fairburn, 2007). Using the pictorial dot-probe, Shafran et al. (2007) tested (1) whether attention biases for food-, shape- and weight-related pictures are stronger in ED participants compared to healthy, anxious and shapeconcerned controls and (2) whether the strength of attention biases is associated 
with the severity of ED psychopathology. Stimuli included pictures of food, body shape, body weight and animals. ED participants were faster to respond to a dot when it replaced negative food and weight pictures, and they responded slower to a dot when it replaced positive food pictures, compared to anxious controls and women with high, moderate, and low levels of shape concerns. In study 2, but not in study 1, ED participants were significantly faster to respond to the dot when it replaced negative and neutral shape pictures compared to the controls. No bias was found for positive shape stimuli. These findings only partially support the previous findings by Rieger et al. (1998). Shafran et al. (2007) suggested that biases for body shapes might be less robust because the pictures were of other persons and hence not personally relevant. Additionally, their results showed there was a modest relationship between the degree of psychopathology and the extent of attention biases.

Further research showed that with increasing duration of the inter-stimulusinterval from $500 \mathrm{~ms}$ to $2000 \mathrm{~ms}$, the attention bias for food and shape pictures in ED patients disappeared whereas the bias for weight stimuli remained (Lee \& Shafran, 2008). These findings suggest a pattern of cognitive avoidance for food and shape pictures that increases over time. This is in line with the cognitive model, stating that the initial attention-bias for threatening stimuli eventually serves to enable patients to actively avoid these stimuli. Thus, the attention bias might prevent confrontation with the feared stimulus. If there is no exposure to feared stimuli, fear responses will not be able to extinguish, and in that way the ED will persist. Interestingly, Shafran et al. (2008) showed that attention biases for food, weight and shape pictures in ED patients decreased after cognitivebehavioral treatment.

\section{VISUAL SEARCH TASK}

Another method to study attention bias is the visual search task. Treisman and Gelade (1980) devised the first visual search task, in which participants were instructed to locate a simple target (e.g., circle, square) as quickly as possible among an array of several distractors, all with a similar shape but different from the shape of the target. Differences in search performance on the visual search task are proposed to reflect differences in the focus of attention. Hansen and 
Hansen (1988) developed the face-in-the-crowd visual search task in which participants search for an odd face stimulus in a matrix of face stimuli. This adaptation had important advantages. First, if the odd-one-out stimulus is a disorder-relevant stimulus, which is presented among neutral distractors, speeded detection of disorder-relevant information can be measured. Second, by making the odd-one-out stimulus a neutral stimulus, which is presented among disorderrelevant distractors, distraction by disorder-relevant information can be measured (Rinck, Reinecke, Ellwalt, Heuer \& Becker, 2005). The distraction component may arguably be similar to maintained attention (Mogg et al., 2005), or slowed disengagement (Fox, Russo, Bowles, \& Dutton, 2001) components of attention bias. This task thus allows for the investigation of specific mechanisms of attention bias: speeded detection and increased distraction. To our knowledge, so far only one study has applied the odd-one-out visual search task in ED patients (Smeets, Roefs, van Furth \& Jansen, 2008). In this study, ED participants showed evidence of speeded detection of body shape words, but not of increased distraction by shape stimuli compared to healthy controls. The opposite pattern of results was found for food-related words: ED participants showed no evidence of speeded detection of high-calorie food words, but there was increased distraction compared to neutral and low calorie words. The finding that ED patients were more distracted by high-calorie words than controls, was explained by craving (Mogg et al., 2005, Smeets et al., 2008). Indeed, a substantial number of studies have found significant correlations between attention bias for cravingrelated stimuli and levels of subjective craving (e.g., Field et al., 2007; Field, Mogg, \& Bradley, 2005; Franken et al., 2000; Rosse et al., 1997; Rosse, Miller, Hess, Alim, \& Deutch, 1993). In a next study, Smeets, Roefs, and Jansen (2009) experimentally induced craving and a causal link between induced chocolate craving and a bias in the distraction component of attention was found. More specifically, when brought to an elevated state of chocolate craving, chocoholics showed more distraction by chocolate than in the absence of such increased chocolate craving state. This study shows that the suggested relationship between craving and attention bias only holds true for the specific distraction component and not for speeded detection. 


\section{EYE TRACKING}

Changes in attention usually are directly related to eye movements (Henderson, Pollatsek, \& Rayner, 1989; Rayner, 1998). Eye tracking has several advantages over reaction time tasks. It provides a more direct indication of attention bias, and it allows not only to measure the initial detection of a stimulus, but also changes in the direction of attention. Also the maintenance of attention on relevant stimuli can be directly assessed by measuring the fixation duration on the stimulus.

Eye tracking studies show that ED patients tend to focus on dissatisfying body parts (Freeman, Touyz, Sara, Rennie, Gordon and Beumont, 1991; Jansen, Nederkoorn \& Mulkens, 2005). Jansen et al., (2005) showed that ED patients allocate their attention more toward their self-identified unattractive body parts than to their self-identified attractive body parts. When looking at the bodies of other persons, the ED patients paid most attention to the other's attractive body parts than to the other's unattractive body parts (upward comparison). Healthy controls however did exactly the opposite; they looked more at their own attractive body parts compared to their own unattractive body parts, and they attended more to the other person's unattractive parts than to the other's attractive body parts (downward comparison).

In an additional study, Jansen, Smeets, Nederkoorn and Martijn (2006) showed that control models had a strong positively biased perception of their own attractiveness whereas ED patients lack this self-serving bias. The cognitive processing in ED patients might have caused this a lack of a self-serving bodyimage bias, since they focus their attention on body parts that are evaluated as unattractive by themselves whereas healthy controls do the opposite. Mulkens and Jansen (2009) demonstrated that increased attention for appearance leads to increased body dissatisfaction in vulnerable participants (highly body dissatisfied participants) whereas healthy controls show increased body satisfaction after increased attention.

It can be concluded that, in line with the cognitive model of EDs, EDs are characterized by an attention bias for high calorie foods and bodies. Even if Stroop studies measure biases in attention, they do not clearly indicate how one's attention exactly is biased; is the attention directed at specifically thin bodies, fat bodies, the own body, attractiveness or unattractiveness, and so on. What 
cognitive processes are involved? And do high calorie food biases and body biases reflect identical cognitive processes? Use of the dot probe task suggests that early attention of ED patients is specifically biased towards negative food and body stimuli. The visual search task showed that body stimuli elicit a bias in speeded detection. This task also showed increased distraction by tasty high calorie food stimuli. It was further demonstrated that craving leads to an attention bias for high calorie food stimuli - more specifically increased distraction. Eye tracking studies demonstrate that the way one inspects bodies is causal to body (dis)satisfaction. EDs specifically focus on their own negatively evaluated body parts, thereby inducing greater body dissatisfaction.

\section{INTERPRETATION BIAS}

The interpretation bias refers to the tendency to interpret ambiguous stimuli in a disorder-relevant way; in EDs ambiguous stimuli are expected to be interpreted as weight- or shape-related, and in a negative way, especially when these stimuli refer to the patients themselves. Ambiguous scenarios such as: "Two friends are giggling and whispering behind you. What do you think they are saying?" are presented. It is assessed how the participant interprets each scenario and whether the interpretation relates to body weight or - shape, either in an open-ended or a forced-choice format.

Cooper (1997) showed that ED patients responded more often with a weight and shape interpretation compared to the healthy controls, both in open-ended and forced-choice negative outcome scenarios referring to themselves. ED patients responded more with positive weight and shape interpretations when the scenarios related to others. Thus, ED patients judge weight and shape to be a more likely explanation for events with a negative outcome and referring to themselves (Cooper, 1997; Morrison, Waller \& Lawson, 2006). Jansen, Smeets, Boon, Nederkoorn, Roefs, \& Mulkens (2007) showed that this was also true for overweight and obese children. Williamson, Perrin, Blouin and Barbin (2000) found that ED patients are able to change interpretations in shape-related situation after explicit instructions. A relevant question for future research is whether retraining interpretations into more positive ones leads to less ED symptoms. It can be concluded that, in line with the cognitive model, EDs are 
characterized by a self-blaming style, in which they judge weight and shape to be a most likely explanation for negative ambiguous events related to the self. Interestingly, Williamson et al. (2000) showed that it is possible to experimentally manipulate the interpretation bias in ED patients; they demonstrated that interpretations could be made less biased, that is less shape-related (Williamson et al., 2000).

\section{MEMORY BIAS}

A memory bias refers to the tendency to recall disorder-specific information more easily. The cognitive model predicts that food, weight and shape information will be more readily encoded in memory and more easily accessed in recall by ED patients. In general, two types of memory are distinguished: explicit and implicit. Explicit memory is characterized by conscious recollection or recognition of a previous event or experience. In contrast, implicit memory is exhibited when prior experience facilitates or primes performance on a task, without conscious recollection of the experience.

Research has found convincing support for the existence of an explicit memory bias in ED patients but data for an implicit memory bias are equivocal (Hermans, Pieters \& Eelen, 1998; Hunt \& Cooper, 2001; King Polivy \& Herman, 1991; Sebastian, Wiliamson \& Blouin, 1996; Tackan, Caglan, Topcuoglu \& Yucul, 2008; Pietrowsky, Krug, Fehm \& Born, 2002; Sebastian, Williamson, \& Blouin, 1996; Suslow et al., 2004). Studies using paradigms like the cued and free recall task and the directed forgetting paradigm, indicate that EDs show specific explicit ED related memory biases: EDs show a memory bias for food-, weight- and shape-related words and not for general emotional words (Hermans et al., 1998; Hunt \& Cooper, 2001; King, et al.,1991; Sebastian et al., 1996 ; Suslow et al., 2004),

It was also found that the memory bias for high caloric foods was independent of food deprivation in AN participants but not in BN participants (Hunt \& Cooper, 2001; Pietrowsky et al., 2002; Suslow et al. 2004).

To date, as far as the present authors know, only two studies have tested implicit memory in ED patients (Hermans et al., 1998; Johansson, Ghaderi, Hallgren \& Andersson, 2008). Hermans et al. (1998) used a word-stem completion task but 
found no differences between AN participants and controls. However, Johanson et al. (2008) argued that the word-stem completion task is not a valid measure of implicit memory, because explicit memory can be used to complete the task. A more valid measure is Jacoby's white noise task (Jacoby, Allen \& Larwin, 1988). In the white noise task, participants listen to sentences and repeat them out loud. Subsequently, these sentences are presented again, but now they are intermixed with new sentences not previously heard along with background noise that varies in intensity. The participant is instructed to judge the intensity of the background noise. Participants rate noise as less loud for sentences previously heard compared with new sentences, suggesting implicit memory (Jacoby et al., 1988). In agreement with the implicit memory bias hypothesis, Johanson et al. (2008) showed that ED participants rated background noise for food and shape sentences being less loud, compared to neutral sentences. In conclusion, EDs show an explicit memory bias for food, weight and shape information, and there are some indications for an implicit memory bias in EDs also (but see Hermans et al., 1998).

\section{GENERAL IMPAIRMENTS IN COGNITIVE PROCESSING}

In addition to studying the biases proposed by the cognitive model, general processing impairments not specifically related to food, weight or shape, have also been investigated. The general cognitive processes of interest are: (1) set shifting, (2) central coherence, and (3) decision making. First, cognitive set shifting is considered, which refers to the ability to move back and forth between multiple tasks, operations or mental sets and is a major component of executive functioning. Problems in set shifting may manifest either as cognitive inflexibility (e.g., concrete and rigid approaches to problem solving) or response inflexibility (e.g., stereotyped behaviors) and have been associated with EDs (Tchanturia, Anderluh et al., 2004). A systematic meta-analysis shows that both $B N$ and $A N$ participants have problems performing a wide range of cognitive set shifting tasks, for example, the Wisconsin Card Sorting Task (Roberts, Tchanturia, Stahl, Southgate \& Treasure, 2007). Furthermore, it was shown that weight recovery in AN participants did not improve cognitive set shifting, indicating that it is a trait and not a state marker of AN (Tchanturia, Morris et al., 2004). Proposing a 
genetic basis for impaired cognitive set shifting, Holliday, Tchanturia, Landau, Collier and Treasure (2005) found more set-shifting difficulties in healthy sisters of AN patients than in unrelated healthy women. The implication of these findings might be that a therapy improving cognitive flexibility and performance on set shifting tasks in ED patients is beneficial in treatment (Tchanturia, Davies \& Campbell, 2007).

The second cognitive ability hypothesized to be impaired in ED is central coherence. A weak central coherence refers to enhanced detailed processing, accompanied by a limited ability to understand context or to 'see the big picture'. This causes information to be processed in parts, rather than as a whole, which impairs global thinking. Central coherence has been recognized as playing an important role in autism spectrum disorders, and it has now been suggested to also be related to the development of EDs (Happe \& Frith, 2006; Lopez, Tchanturia, Stahl \& Treasue, 2009). Weak central coherence might explain the preoccupation with details and rules observed in many ED patients.

AN patients were also found to have a weaker theory of mind compared to healthy controls (Russel, Schmidt, Doherty, Young \& Tchanturia, 2009; Harrison, Sullivan, Tchnaturia \& Treasure, 2009). Theory of mind refers to the cognitive ability to understand the internal states of others and a weak theory of mind is characteristic of autism. However, Oldershaw, Hamboork, Tshanturia, Treasure and Schmidt (2009) demonstrated that recovered AN patients performed significantly better than currently ill AN patients when inferring emotions during a theory of mind task, showing that impaired theory of mind is likely to be caused by self-induced starvation. In a study by Lopez et al. (2008), AN patients and healthy controls completed several tests measuring visuospatial and verbal aspects of central coherence (Rey-Osterrieth Complex Figure Test, Embedded Figures Test, Homograph Reading Test and Sentence Completion Task). Results showed that the AN group scored significantly better on tests requiring local processing and worse on global processing tasks. There was no association between performance and depression, anxiety or degree of starvation. However, weak central coherence was correlated with the number of obsessive-compulsive traits. This fits nicely with the idea of a weak central coherence explaining the anorectic preoccupation with details and rules.

A third cognitive ability that is studied in ED is decision making. ED patients show impaired decision-making (Boeka \& Lokken, 2006; Brand, Franke-Sievert, 
Jacoby, Markowitsch, \& Tuschen-Caffier, 2007). BN patients performed significantly worse on the lowa Gambling Task (IGT) and the Game of Dice Task, compared to a control group (Cavendini, Zorzi, Bassi, Gorini, Baraldi, Ubbiali \& Bellodi, 2005). There was a significant negative correlation between performance and bulimic symptoms, independent of depressive symptoms (Boeka \& Lokken, 2006; Brand et al., 2007; Cavendini et al., 2004) It was also found that AN patients who had better decision-making abilities at the start of a treatment showed significantly greater improvement in nutritional status (Cavendini et al., 2005).

\section{MANIPULATION OF COGNITIVE PROCESSES}

It is concluded that ED patients are characterized by cognitive biases and some errors in general cognitive processing. It is not entirely clear whether these biases and impairments in cognitive processes are causes, consequences, or an epiphenomenon of EDs. What happens when the biases are manipulated, for example by attention retraining? In this section we discuss some recent studies on the retraining of attention bias and the cognitive modulation of food reward processes.

\section{RETRAINING ATTENTION BIAS}

It was discussed above that ED patients show an attention bias for their own unattractive body parts. A first question is whether this way of looking is causal to greater body dissatisfaction. To test causality, Smeets, Jansen, Lindelauf \& Roefs (2010) experimentally manipulated the way of looking in healthy participants, and measured its effects on body satisfaction. Results showed that the way one looks at one's own body causes changes in body satisfaction: healthy participants who were trained to attend to their self-defined unattractive body parts showed significantly decreased body satisfaction after the training. It was also showed that slightly body dissatisfied female students who were trained in attending one's own most attractive body parts showed a significant increase in body satisfaction after the training (Smeets et al., 2010). Thus, the way one inspects bodies is 
causal to body (dis)satisfaction. Retraining the attention bias for negatively evaluated body parts into increased attention for positively evaluated body parts, that is the way healthy females look, was found to be beneficial; this way of looking increased body satisfaction (Smeets et al., 2010). It is of great interest to find out whether such an attention retraining is clinically useful.

Another retraining study focused on inhibitory control (Houben \& Jansen, 2010). It was studied whether strengthening inhibitory control can increase resistance to high calorie food temptations. Chocolate cravers were trained to inhibit their responses to chocolate stimuli, which led to significantly reduced chocolate consumption. These findings suggest that strengthening inhibitory control might be an effective strategy to help regain control over food intake.

\section{MODULATION OF FOOD REWARD PROCESSING}

Food reward stimulates eating behaviour (Toates, 1986). The incentive salience of food is evaluated in the dopaminergic corticomesolimbic circuitry and motivates eating behavior in the absence of energy deficits (Berridge, 2004; Bindra, 1978; Bolles, 1972). Whether one actually eats - or not - depends on the interaction between food reward and cognitive control (Appelhans, 2009). Is it possible to manipulate one's cognitive control in such a way that one is better able to cope with an environment rich in palatable, readily available high calorie foods? It was therefore studied whether healthy lean women are able to modulate food reward processing using different types of cognitive control strategies (Siep et al., 2010). Participants were instructed 1) to suppress food palatability thoughts (i.e, do not think about how tasty the food is), 2) to apply cognitive reappraisal (e.g., think about the health consequences of eating it, like gaining weight), and 3) to up-regulate thoughts of food palatability (e.g., think about how good the food smells and tastes). It was investigated whether the three cognitive control strategies changed self-reported food cravings and associated food reward activity in the brain as assessed by functional Magnetic Resonance Imaging (fMRI). Both the cognitive reappraisal and suppression manipulations decreased self-reported food cravings compared to up-regulation. The fMRI data suggest that these strategies rely on different neural substrates. Cognitive reappraisal decreased activity in the fusiform gyrus (FG) compared to up-regulation and suppression, 
but did not differ from passive viewing in mesocorticolimbic regions. The FG is involved in the processing of visual cues and their reward values, and determines future reward actions (Murray and Izquirdo, 2007). The brain responses during cognitive reappraisal suggest that this strategy prevents food cues from eliciting further reward processing. This proposition is in line with the definition of cognitive reappraisal (Gross \& John, 2003) as 'thinking about the emotion eliciting cue in a way that changes its emotion impact'. Suppression decreased activity in the ventral striatum (VS) and ventral tegmental area (VTA), and increased activity in the lateral orbitofrontal cortex (OFC) and anterior prefrontal cortex (aPFC) compared to up-regulation and suppression. Although suppression successfully inhibited activity in the VA and VTA, the increased prefrontal cortex activity suggests that this strategy requires increased mental effort compared to cognitive-reappraisal. As expected, up-regulation increased activity in the mesocorticolimbic circuitry. Together these findings show that people can actively up and down-regulate mesocorticolimbic food reward processing, and that craving covaries with the use of cognitive control strategies.

In line with this, one might speculate that AN is associated with extremely successfully applied cognitive strategies to decrease food reward processing. A cognitive effect of rigid dieting might be the down-regulation of food reward activity in the brain. AN might develop when highly controlled, rigid and obsessively dieting adolescent females overcome the natural rewarding value of food (Pinel, Assanand, \& Lehman, 2000). To test the hypothesis of downregulated food reward activity in AN, AN patients were instructed to evaluate the palatability of high and low calorie foods and at the same time corticomesolimbic food reward activity was measured (Siep et al., 2010). Furthermore, it was studied what would happen in the dorsolateral prefrontal cortex (dlPFC) when Anorexia Nervosa patients focused their attention on a neutral part of the picture while they were simultaneously presented with food pictures. The dIPFC is an area involved in successful self-control (Hare, Camerer, \& Rangel, 2009). Interestingly, it was shown that AN patients evaluating high calorie foods fail to activate two important regions of the corticomesolimbic food reward circuitry: the anterior insular cortex (AIC) and caudal Anterior Cingulate Cortex (CAAC). Both the AIC and CACC are involved in craving (Craig, 2002, 2003; Naqvi, Rudrauf, Damasio, \& Bechara, 2007) and in the motivation of reward-related behavior (Walton et al., 2009). Nagvi et al., (2007) showed that 
smokers who acquire insula damage are likely to stop smoking quite easily. In line with this, it could be hypothesized that a decreased responsiveness of AIC in AN patients allows them to easily stop eating. The AN patients did show increased activity in the right anterior OFC during the palatability evaluation of low calorie foods, supporting the hypothesis of successfully downregulating the food reward processing. In a meta-analysis of neuroimaging studies Kringelbach and Rolls (2004) concluded that the anterior OFC is involved in the processing of abstract reinforcers such as money. It was concluded that AN patients might evaluate low calorie foods as rewarding but, in contrast to healthy people, their food evaluations appear not to be intuitive (Kaye, Fudge, \& Paulus, 2009) or based on palatability (Roefs et al., 2005). To test the proposition of increased dIPFC activity in AN, participants were instructed to focus their attention on a neutral cue while high calorie foods were simultaneously presented in an unattended part of the stimulus display. This led to a strongly activated dIPFC in AN, but not in healthy controls (Siep et al., 2010). These findings support earlier ones that show dorsolateral prefrontal cortex hyperactivity in Anorexia Nervosa, indicating a relatively quick and automatic activation of increased self-control when confronted with high calorie foods.

\section{COGNITIVE THERAPY}

According to the cognitive model of EDs, ED patients use their own body weight and shape as the predominant factors for inferring personal value. Dysfunctional weight and shape beliefs flow from cognitive structures referred to as schemata. Activation of these schemata produces systematic errors in information processing, like attention biases, interpretation biases and memory biases for food and body stimuli. In this way a negative self-perception is maintained. ED patients not only show biases in the processing of food-, weight- and shaperelated cues, some recent studies showed that they also demonstrate errors in general cognitive abilities like set shifting, central coherence, decision making, emotion recognition and self-regulation.

Cognitive Behaviour Therapy (CBT) is up to now the most effective treatment for EDs (Wilson, Grilo \& Vitousek, 2007). During the cognitive intervention of CBT, dysfunctional cognitions are challenged and a change in thinking is strived for. 
The assumption is that when thinking changes, emotions will be more positive and behavior will be less symptomatic. Future studies might focus on the question whether $\mathrm{CBT}$ might still improve from incorporating attention retraining to improve body image, attention retraining to strengthen inhibitory control, techniques to change the errors in general cognitive abilities, and training in the manipulation of food reward processing. This of course requires large scale randomized clinical trials with a so termed additive design. But we would like to contend that the development of progressively efficacious treatments for EDs just as much requires elegant experimental studies such as outlined in the present chapter, experiments that increase our knowledge of the basic mechanisms underlying eating psychopathology. 
General Discussion 


\section{GENERAL DISCUSSION}

This dissertation discussed the study of mesocorticolimbic processing, using functional magnetic resonance imaging (fMRI) to investigate the influence of calorie content, hunger, attention and cognitive strategies on food reward activity in healthy people and impaired food reward processing in Anorexia Nervosa patients. The focus on food reward as an important neurobiological mechanism regulating (ab)normal eating behaviour, was inspired by the incentive motivation theory (Bindra, 1978; Bolles, 1972; Toates, 1986). This theory posits that through processes of associative learning, food acquires a motivational value that drives eating behaviour, even in the absence of a clear biological need. The study of mesocorticolimbic food reward processing in the brain therefore holds promise of providing important insights into (ab)normal eating behaviour.

\section{SUMMARY OF THE FINDINGS}

Chapter 2 discusses the influence of calorie content and food deprivation on reward processing. FMRI results indicated that satiated participants showed increased mesocorticolimbic activity in response to the presentation of palatable low calorie foods. In contrast, the food-deprived participants showed increased activity following the presentation of equally palatable high calorie foods. This finding suggests that calorie content modulates food reward activity independent of palatability, as well as interacts with homeostatic needs. This finding is in line with the optimal foraging theory (MacArthur \& Pianka, 1966), which states that organisms forage in such a way as to maximize their energy intake. Additional results showed that the experimental manipulation of attention focus had a large impact on activity in both the amygdala and the orbitofrontal cortex, in that both brain regions showed significant activity only when the task required participants to attend to the palatable taste, smell and texture of a presented visual food cue, but not when they were required to attend to a neutral aspect of the same food cue. Although these findings indicate that a dissociation between amygdala and orbitofrontal cortex functioning cannot be made based on differences in attention requirements, they do suggest that research on how attention modulates mesocorticolimbic activity might prove especially insightful in the study of food 
reward processing.

Chapter 3 presents an fMRI study in which food reward processing in Anorexia Nervosa patients was investigated. The results show that when high and low calorie foods are evaluated, this increases activity in a network of brain regions, including those implicated in reward processing (i.e., the anterior insular cortex and caudal anterior cingulate cortex) in the hungry and satiated healthy control participants, but not in the Anorexia Nervosa group. Apparently, food reward processing is reduced in Anorexia Nervosa patients, even compared to satiated participants, when food stimuli are consciously evaluated. These findings could not be attributed to the possible confounding effect of anemia, showing similar or even increased activity in the neutral condition for the Anorexia Nervosa patients, compared to the healthy controls. Interestingly, when food cues were presented but participants did not explicitly attend to them, food reward processing in AN patients was observed as opposed to the healthy satiated but not hungry controls. This increased food reward processing was concurrent with increased activity in the right dIPFC and the left aTemp. Clearly, when Anorexia Nervosa patients are responsive to food temptations, as can be derived from increased food reward processing, they display an immediate compensatory increase in inhibitory self-control. But note that this heightened self-control was specific for the incidental exposure to the high calorie food pictures.

The fMRI study presented in Chapter 4 examined the effects of up-regulation, suppression and cognitive reappraisal on mesocorticolimbic activity in healthy female participants. Self-report results indicated that up-regulation increased food craving compared to the other two conditions, but that there was no difference in craving between the suppression and cognitive reappraisal strategy. Corroborating self-reports, the neuroimaging results showed that up-regulation increased activity in mesocorticolimbic regions. Contrary to our hypothesis, suppression more effectively decreased activity in the core of the mesocorticolimbic circuitry (i.e., ventral tegmental area and ventral striatum). However, results also show that suppression led to increased activity in the prefrontal cortex, supporting the proposition that suppression requires more selfregulatory effort than cognitive reappraisal. 


\section{IMPLICATIONS \& FUTURE RESEARCH}

The results of the first fMRI study described in Chapter 2 indicate that high calorie foods are more rewarding than low calorie foods when people are food deprived. This finding has an important clinical implication. Energy restriction is one of the most frequently applied strategies in the treatment of excessive weight. The finding of an increased reward value for high calorie foods when hungry gives a possible explanation of why dietary restriction may be difficult: food restriction increases the attraction of forbidden fruits. It can be hypothesized that diets limited in their energy content but with good satiating properties (e.g., highprotein diets) could be an effective tool in weight-loss treatments. Supporting this notion, Weigle et al. (2005) showed that an increase in dietary protein from $15 \%$ to $30 \%$ of energy and a reduction in fat from $35 \%$ to $20 \%$, at a constant carbohydrate intake, produced a sustained decrease in ad libitum calorie intake and resulted in significant weight loss. They sequentially assigned one group of 19 participants to the following sequential diet regime: (1) two weeks of a weight maintenance diet (15\% of energy as protein, $35 \%$ as fat, and $50 \%$ as carbohydrate); (2) two weeks of a diet with a similar amount of calories as the weight maintenance diet, but with more proteins and less fat (30\% of energy as protein, $20 \%$ as fat, and $50 \%$ as carbohydrate); or (3) 12 weeks of an ad libitum diet with the same macronutrient composition (30\% of energy as protein, $20 \%$ as fat, and $50 \%$ as carbohydrate). Results showed that the participants successfully maintained a stable body weight during the first two weeks of the diet. Participants reported a clear decrease in hunger and an increase in fullness during weeks 3 and 4 of the study, after the transition to the high-protein diet. This increase in satiety was confirmed by a decrease in spontaneous calorie intake of $494 \mathrm{kcal}$ per day within $24 \mathrm{hrs}$ of starting the ad libitum diet phase of the study and the maintenance of a significant decrease in spontaneous calorie intake relative to baseline through the end of the study. This sustained decrease in spontaneous calorie intake resulted in a constant rate of weight loss during the 12-week ad libitum high-protein diet, which amounted to $4.9 \mathrm{~kg}$ lost by the end of the study. These results suggest that increasing protein intake should result in a larger weight loss, due to its satiating properties, and related decreases in high calorie food reward value, compared to diets that focus on restriction of calorie intake only. Although the present fMRI studied the effects of short-term hunger on 
food reward processing, research suggests that long-term fasting decreases the desire to eat, hunger and prospective consumption for non-obese people, whereas these appetite sensations tend to increase slightly for the obese (Oh, Kim \& Choue, 2002). Further research is necessary to more fully investigate the different effects of food deprivation on mesocorticolimbic processing in nonobese and obese people.

Although the manipulation of attention focus in the fMRI study discussed in Chapter 2 did not result in the hypothesized functional dissociation between the amygdala and the orbitofrontal cortex, this manipulation had another interesting effect. The attention focus manipulations showed that regions of the mesocorticolimbic circuitry were only significantly activated when the participants explicitly evaluated foods. This finding suggests that mere presentation of foods does not automatically result in mesocorticolimbic activity. This finding leads to several interesting hypotheses. First, participants were explicitly instructed to evaluate food palatability when satiated. It can be hypothesized that in a "real world" situation, healthy satiated people usually do not evaluate food palatability, because food consumption is biologically less relevant for them at that moment. By instructing the satiated healthy participants to evaluate foods, they were forced them to think "in a hungry way". This might also explain why the effects of food deprivation on brain activity were too small to be detected by the random effects analysis. In this case, passive viewing might have resulted in larger effects. However, passive viewing has the disadvantage of not knowing what the participants are thinking of. For example, showing a picture of food without instruction might have reminded the participant of the fact that she still needs to do her grocery shopping before she goes home. Measuring this type of brain activity would not have been relevant for the hypothesis under investigation. This dilemma nicely illustrates the decisions fMRI researchers are faced with. When deciding on the appropriate design, one should carefully consider the cognitive changes caused by the task instructions and decide whether these cognitive changes reflect the way people would be naturally tend to think in such a situation. If not, a passive task or a task that allows cognitions to change more freely may be better suited.

The discussion about the influence of task instructions on mesocorticolimbic brain activity led to other interesting hypotheses. First, following the results of the first fMRI study, it was concluded that the effect might have been larger if satiated 
persons had not been instructed to evaluate food palatability, because they would normally not do so. It would be interesting to test whether overweight and obese people do evaluate food palatability when satiated, compared to healthy lean participants. Activation of the mesocorticolimbic by frequent palatability evaluation of food even when satiated could explain why overweight and obese people eat too much. This hypothesis is currently under investigation in our lab (Frankort et al., In Prep).

A second hypothesis that resulted from the discussion about the influence of task instructions on mesocorticolimbic activity is that the manipulation of mindset (i.e., a mental attitude) should have a large impact on mesocorticolimbic food reward processing. For example, manipulating participants to think like a culinary chef when passively presenting food stimuli will result in a larger increase in mesocorticolimbic food reward activity, compared to instructing participants to think like a dietician. This proposition is supported by a study showing that automatic evaluations of foods were based on palatability in a restaurant-focus condition, whereas they were based on health in a diet-focus condition (Roefs et al., 2006). Although the suggestion that mind affects food evaluation and related mesocorticolimbic activity might seem self-evident, this hypothesis is especially relevant in light of the current debate on the role of the toxic Western environment (i.e. an environment where highly palatable foods are easily accessible; Brownell, 2005) in the increasing prevalence of obesity. After all, especially in these environments, fast-food companies spend large amounts of money to influence people's mindsets, trying to increase food intake. The suggestion that changes in people's mindsets might lead to changes in food intake is further supported by the finding of the fMRI study described in Chapter 4 , showing that changes in cognitive strategies applied during food evaluation led to corresponding differences in mesocorticolimbic activity and self-reported food craving.

In line with the hypothesis of decreased food reward processing in AN patients (Kaye et al., 2009; Pinel et al., 2000), results of the fMRI study described in Chapter 3 showed decreased anterior insular cortex and caudal anterior cingulate cortex processing in Anorexia Nervosa patients as compared to healthy women. This finding suggests that a mechanism that triggers eating behaviour in healthy people is impaired in Anorexia Nervosa patients. This finding might have implications for the effective treatment of eating behaviour in Anorexia Nervosa 
patients. Current treatments for Anorexia Nervosa mainly focus on restoration of weight under strict dietary supervision and changing weight- and shape-related cognitions (NICE, 2009). However, these treatments lack the support of research confirming their effectiveness (Wilson \& Shafran, 2005), which is supported by the finding that current treatments result in full recovery in only $46 \%$ of Anorexia Nervosa patients (Steinhausen \& Weber, 2009). To increase the effectiveness of Anorexia Nervosa treatments, and in line with the present research findings, it should be investigated whether methods that increase mesocorticolimbic food reward activity can improve treatment outcomes. A method that might be effective in increasing mesocorticolimbic activity in Anorexia Nervosa, as inspired by the results of the third fMRI study, is cognitive modulation. For example, using advanced methods like real-time fMRI (DeCharms, 2008; Weiskopf et al., 2007), one could train Anorexia Nervosa patients to increase activity in the mesocorticolimbic circuitry while food pictures are presented, using up-regulation strategies, starting with less threatening low calorie stimuli and eventually progressing to high calorie stimuli. Future research is needed to test the effectiveness of methods like real-time fMRI up-regulation strategies in restoring normal eating behaviour in Anorexia Nervosa patients.

Kaye et al. (2009) suggest that the decrease in mesocorticolimbic activity is the result of an abnormal increase in dorsolateral prefrontal cortex "control" activity. The present results supported this proposition: instructing participants to focus on a neutral stimulus aspect while a high calorie food was presented strongly activated the right dorsolateral prefrontal cortex in Anorexia Nervosa patients but not in healthy control participants. These findings suggest that an effective increase in mesocorticolimbic food reward processing might only be accomplished when dorsolateral activity is inhibited, for example, using transcranial direct current stimulation (Hecht, 2010). However, the temperament and personality traits that are hypothesized to be related to the increased dorsolateral prefrontal activity might also have positive aspects. It is suggested that the traits resulting from increased dorsolateral prefrontal cortex activity (including attention to detail, concern about consequences and a drive to accomplish and succeed) contributes to the clinical observation that many individuals who recover from Anorexia Nervosa do very well in life (Kaye et., 2009). Therefore, permanent inhibition of the dorsolateral prefrontal cortex might not be necessary; instead, it can be suggested that the need to control should be 
diverted away from food and weight regulation and toward a more beneficial goal (e.g., career, study, etc.).

Results of the third fMRI study discussed in Chapter 4 showed that women can successfully modulate activity in the mesocorticolimbic circuitry by application of cognitive control strategies. This finding is in line with the proposition that eating behaviour is the product of an interaction between incentive motivation processes and cognitive control (Appelhans, 2009). This is an interesting finding, as abnormal mesocorticolimbic food reward processing plays an important role in abnormal eating behaviour (Rothemund et al., 2007; Stice, Spoor, Bohon, \& Small, 2008; Stoeckel et al., 2008; Wagner et al., 2007; Wang et al., 2001). Research indicates that the lack of self-control might be caused by a decrease in inhibitory prefrontal activity (Volkow et al., 2008) that in turn results in impulsive eating behaviour (Nederkoorn et al., 2006). Future research is necessary to determine the causal relationship between decreased prefrontal control processes and increased mesocorticolimbic activity in relation to overeating.

Results of the third fMRI study indicate that cognitive reappraisal requires less mental effort, as indicated by decreased prefrontal activity, compared to suppression. This finding suggests that cognitive reappraisal might be a more efficient cognitive control strategy for obese people who have low prefrontal cortex activity (Volkow et al., 2008). Furthermore, it would be interesting to explore the possibilities of cognitive regulation strategies in changing eating behaviour. The fMRI study described in Chapter 4 showed that cognitive strategies influence mesocorticolimbic processing. A follow-up study is necessary to test whether cognitive control strategies change food intake, as well. For example, it would be interesting to see whether training participants to successfully apply cognitive reappraisal strategies while presented with their favourite palatable foods results in decreased food intake. If cognitive control strategies can indeed successfully change food intake, this would make them an attractive tool in changing eating behaviour in those who eat too much or too little. 


\section{CONCLUDING REMARKS}

This dissertation shows that the investigation of mesocorticolimbic food reward processing provides important insight into factors that influence (ab)normal eating behaviour. For example, the present results showed that short-term food deprivation causes high calorie foods to become more rewarding, which has important implications for those who are trying to lose weight. Furthermore, it was shown that explicit evaluation of food palatability activates the mesocorticolimbic circuitry, which led to an investigation of the role of cognitive control strategies in modulating food reward processes. Indeed, the present findings indicate that women can modulate mesocorticolimbic activity by using techniques like up-regulation, suppression and cognitive reappraisal. This finding is especially relevant, considering the findings of decreased food reward processing in Anorexia Nervosa patients and increased food reward processing in obese people (Kaye et al., 2009; Rothermund et al., 2007; Stoeckel, 2008). Further research needs to be done to investigate whether training patients in the successful application of cognitive control strategies, possibly using advanced techniques like real time fMRI, leads to long-term changes in mesocorticolimbic activity and subsequent food intake.

If I had the funding for five years of research I would like to test whether...

- Changing participants' mindsets from health-centered to palatability-centered leads to increased activity in the mesocorticolimbic food reward circuitry.

- Decreasing dIPFC activity, for example using direct current stimulation, results in a decreased need to control and related symptom severity in Anorexia Nervosa patients.

- Obese people are less successful in decreasing mesocorticolimbic activity by the application of cognitive control strategies, compared to lean control participants.

- The increased activity in the mesocorticolimbic circuitry of obese people is the result of decreased prefrontal cognitive capacity.

- Training normal, overweight and obese participants to successfully apply cognitive control strategies when presented with palatable foods will not only decrease activity in the mesocorticolimbic circuitry, but also decrease subsequent food desire and intake. 
Summary 


\section{SUMMARY}

Many people believe that their eating behaviour is mainly driven by homeostatic processes. However, if eating behaviour was indeed controlled by homeostasis alone, most people would only eat when the body was in need of nutrients. Observations of eating behaviour, for example, in restaurants or at parties, tell us this is not necessarily true. In fact, many people will eat when presented with highly palatable foods, independent of how hungry they are. This indicates that there is an additional process, besides homeostasis, that drives eating behaviour. To explain and describe this second process, researchers have formulated the incentive motivation theory (Berridge, 2004; Bindra, 1978; Bolles, 1972; Toates, 1986). This theory proposes that food can acquire incentive salience through processes of associative learning, triggering and driving eating behaviour, even in the absence of homeostatic deficits. The term food reward was adopted by researchers to refer to this type of eating behaviour and related processes (Kelley \& Berridge, 2002; Wise, 2006). For a long time any qualitative food reward research was difficult due to its subjective nature and was largely dependent on self-reports. This changed with the invention of neuroimaging methods. By studying changes in brain activity known to be involved in reward processing (i.e., the mesocorticolimbic circuitry), researchers recently have started to gain insight into variables that change the rewarding values of food, which might therefore also influence human eating behaviour.

Chapter 1 gives an historical overview of food reward research, showing how studies of eating behaviour shifted from a mainly homeostatic perspective to a view of eating behaviour that also included food reward processes. The incentive motivation theory is discussed (Berridge, 2004; Bindra, 1978; Bolles, 1972; Toates, 1986), and the term food reward is defined. Functional magnetic resonance imaging (fMRI) has been the primary research method in the studies described in this dissertation and therefore a brief explanation of fMRI and its application in food reward research is provided. The study of mesocorticolimbic food reward processing has also inspired researchers to develop theories on how these processes might be impaired in people with abnormal eating behaviour, like Anorexia Nervosa patients. A short review of these theories is presented. Finally, it is argued that the investigation of cognitive control strategies that can influence mesocorticolimbic activity might provide important insights into how to 
alter impaired food reward processing. In line with these propositions, the aims of the present dissertation are presented: to study the influence of calorie content, hunger, attention and cognitive strategies on mesocorticolimbic activity using functional magnetic resonance imaging (fMRI) and to investigate the hypothesized decrease in food reward processing in Anorexia Nervosa patients. Chapter 2 presents an fMRI study studying the effects of calorie content, food deprivation and attention on mesocorticolimbic food reward processing in healthy women. The interest in calorie content in food reward processing was inspired by the optimal foraging theory (MacArthur \& Pianka, 1966, which states that organisms forage in such a way as to maximize their energy intake, which would provide evolutionary benefits. Indeed, several studies indicate that people easily overeat high calorie foods. However, it is difficult to separate the effects of calorie content and palatability, as the two go often hand in hand (Green, Wales, Lawton, \& Blundell, 2000). Therefore, the first aim of this study was to investigate the influence of calorie content on food reward processing, while keeping palatability equal. The second aim of this study was to examine the effect of food deprivation on food reward processing. The incentive motivation theory suggests that although homeostatic deficit signals are not sufficient to drive motivated behaviour directly, they do magnify the hedonic impact and reward value of foods, as is indicated by the saying "Hunger is the best spice". In addition, research indicates that an experimentally controlled trial of dieting increases cravings for high calorie foods (Gilhooly et al., 2007). The third and final aim was to test whether a functional dissociation could be made between food reward activity in the amygdala and the orbitofrontal cortex, based on differences in attention requirements. Although both structures have been implicated in the representation of stimulus-reward associations (Baxter \& Murray, 2002; Kringelbach, 2005), considering the efficiency of the brain, it is highly unlikely they perform similar operations. Addressing these three aims, healthy females were presented with high and low calorie food pictures (hypothesis 1) after being either food deprived for 18 hours or provided with a satiating lunch (hypothesis 2), and were instructed to evaluate their palatability. In addition, attention focus was manipulated by directing participants' attention either to the food or to a neutral stimulus aspect (hypothesis 3). FMRI results showed that satiated participants showed increased mesocorticolimbic activity to palatable low calorie foods. In contrast, the food-deprived participants showed increased activity 
following the presentation of equally palatable high calorie foods. This finding proposes that calorie content modulates food reward independent of palatability and interacts with homeostatic needs. This finding is in line with the optimal foraging theory, which states that organisms forage in such a way as to maximize their energy intake. Additional results showed that the experimental manipulation of attention focus had a noticeable impact on activity in both the amygdala and the orbitofrontal cortex, in that both brain regions showed significant activity only when the participants evaluated food stimuli. These findings indicate that a dissociation between amygdala and orbitofrontal cortex functioning cannot be made based on differences in attention requirements. However, these results did suggest that a further investigation of attentional processes on the modulation of reward processing might be especially informative in determining the neural substrates of healthy and pathological eating behaviour.

Anorexia Nervosa patients starve themselves in a world of plenty. Whereas most people struggle to restrain their food intake in the present "toxic" environment, emaciated Anorexia Nervosa patients are successful ascetics who appear to easily refuse the widely available tasty temptations. Why are they so successful in not eating? The study described in Chapter 3 investigated the hypothesized impaired food reward processing in Anorexia Nervosa patients by measuring whole brain activity while instructing Anorexia Nervosa patients and healthy satiated and hungry controls to focus on pictures of high and low calorie foods and to imagine the food's palatability, smell, texture and taste. In contrast to the findings of the previous fMRI study, described in Chapter 2, it was expected that even though Anorexia Nervosa patients have huge homeostatic deficits, mesocorticolimbic activity would be decreased comparable to that of satiated participants (hypothesis 1). Similar to the previous study, attention was manipulated, testing whether food reward processing in Anorexia Nervosa patients would be increased when food stimuli were not explicitly evaluated, comparable to that of healthy controls (hypothesis 2). Lastly, anemia is a frequent complication of Anorexia Nervosa (Cleary, Gaudiani, \& Mehler, 2010), resulting in decreased hemoglobin levels. Because the MRI signal depends on the amount of blood hemoglobin present, a lack of hemoglobin could decrease the intensity of the MRI signal and form a possible confounder when investigating decreases in mesocorticolimbic activity. To control for this, two neutral conditions were added. In the first condition objects were evaluated based on their colour, in the 
second condition objects were presented while directing participants' attention to a neutral stimulus. It was hypothesized that these conditions should not result in differences in neural activity between Anorexia Nervosa patients and healthy controls (hypothesis 3 ). The results show that when high and low calorie foods are evaluated, this increases activity in a network of brain regions, including those implicated in reward processing (i.e., the anterior insular cortex and caudal anterior cingulate cortex) in the hungry and satiated healthy control participants, but not in the Anorexia Nervosa group. Apparently, food reward processing is reduced in Anorexia Nervosa patients, even compared to satiated participants, when food stimuli are consciously evaluated. These findings could not be attributed to the possible confounding effect of anemia, showing similar or even increased activity in the neutral condition for the Anorexia Nervosa patients, compared to the healthy controls. Interestingly, when food cues were presented but participants did not explicitly attend to them, food reward processing in AN patients was observed as opposed to the healthy satiated but not hungry controls. This increased food reward processing was concurrent with increased activity in the right dIPFC and the left aTemp. Clearly, when Anorexia Nervosa patients are responsive to food temptations, as can be derived from increased food reward processing, they display an immediate compensatory increase in inhibitory selfcontrol. But note that this heightened self-control was specific for the incidental exposure to the high calorie food pictures. These findings elucidate why not eating is relatively easy in Anorexia Nervosa: when paying attention to food cues, reward processing is decreased and fronto-temporal correction is not necessary. However, when food cues are unattended, they do elicit reward processing, meaning that Anorexia Nervosa patients still are responsive to food temptations. But when the Anorexia Nervosa food reward circuitry responds to temptation, inhibitory fronto-temporal activity is also immediately increased. This combination of increased reward processing and increased inhibitory activity might enable Anorexia Nervosa patients to resist temptations Many popular diets claim that one can overcome the irresistible temptation of highly palatable foods by actively controlling the way one thinks about food. Testing this claim, the study described in Chapter 4 investigated the capability of three different types of cognitive control strategies to modulate mesocorticolimbic activity and self-reported food cravings: (1) up-regulation, which increases the intensity of innate responses (Gross, 2006); (2) cognitive reappraisal, which 
affects the way one thinks about emotion-eliciting cues and changes their emotional impact (Gross, 2006); and (3) suppression, which can be related to the active inhibition of thoughts (Wenzlaff \& Wegner, 2000) and emotional responses (Gross, 2006). Suppression is a form of emotion regulation that requires self-monitoring and self-corrective action throughout an emotional event. Such monitoring requires a high level of mental effort. In contrast, cognitive reappraisal is evoked early in the emotion-generative process and does not require costly self-regulatory effort, and can thus be maintained for longer durations (Gross \& John, 2003). We hypothesized that cognitive reappraisal in particular would inhibit the mesocorticolimbic activity and associated food craving. Self-report results indicated that up-regulation increased food craving compared to the other two conditions, but that there was no difference in craving between the suppression and cognitive reappraisal strategy. Corroborating self-reports, the neuroimaging results showed that up-regulation increased activity in mesocorticolimbic regions. Contrary to our hypothesis, suppression more effectively decreased activity in the core of the mesocorticolimbic circuitry (i.e., ventral tegmental area and ventral striatum). However, results also show that suppression led to increased activity in the prefrontal cortex, supporting the proposition that suppression requires more self-regulatory effort than cognitive reappraisal. Overall, the results support the contention that appetitive motivation can be modulated by the application of cognitive control strategies, a finding that may be especially relevant for the treatment of pathological eating behaviours.

Chapter 5 presents a review of the main cognitive biases and impairments in cognitive processes that are demonstrated in eating disorders. This chapter discusses whether the manipulation of cognitive biases will change eating disorder psychopathology. Some of the ideas described have been inspired by the fMRI studies presented in this dissertation, illustrating how fMRI research contributes to the understanding of eating disorders and how it might aid in the development of successful treatments.

Chapter 6 summarized the findings of the present dissertation and the implications for further research are discussed. This dissertation shows that fMRI investigation of food reward processing provides important insights into factors that influence (ab)normal eating behaviour. For example, the finding that shortterm food deprivation causes high calorie foods to become more rewarding has important implications for those who are trying to lose weight. Furthermore, 
neuroimaging results indicate that only explicit evaluation of food palatability activates the mesocorticolimbic circuitry, which led to the investigation of the role of cognitive control strategies on food reward possessing. Indeed, the present findings indicate that women can modulate mesocorticolimbic activity, using techniques like up-regulation, suppression and cognitive reappraisal. This finding is especially relevant, considering the findings of decreased food reward processing in Anorexia Nervosa patients and increased mesocorticolimbic activity in obese people (Kaye et al., 2009; Rothermund et al., 2007; Stoeckel, 2008). Further research is necessary to find out whether training patients in modulating mesocorticolimbic activity by successful application of cognitive control strategies, possibly using advanced techniques like real time fMRI, leads to long-term changes in mesocorticolimbic activity and subsequent food intake. 
Samenvatting 


\section{SAMENVATTING}

Velen van ons denken dat het menselijke eetgedrag voornamelijk wordt gereguleerd door homeostatische processen. Zou dit inderdaad zo zijn, dan zouden wij alleen eten wanneer ons lichaam een tekort aan voedingstoffen heeft. Observaties van eetgedrag, bijvoorbeeld in restaurants of op feestjes, laten zien dat dit niet altijd het geval is. Sterker nog, bij aanbod van overheerlijke voeding zullen de meeste mensen eten, of zij nu honger hebben of niet. Deze observaties tonen aan dat er naast homeostatische processen nog aanvullende processen zijn die ons eetgedrag aansturen. Om deze processen te omschrijven en onderzoeken wordt de incentive motivation theorie geformuleerd (Berridge, 2004; Bindra, 1978; Bolles, 1972; Toates, 1986). Deze theorie veronderstelt dat wanneer men in aanraking komt met smakelijke voedingsmiddelen er een associatie wordt geleerd tussen het voedingsmiddel en de beloning die wordt verkregen door consumptie van dit voedingsmiddel. Dit leerproces zorgt ervoor dat bij een volgende confrontatie met het voedingsmiddel, deze een aantrekkingskracht op ons uitoefent die ons aanspoort het voedingsmiddel te eten. Hoe aangenamer het voedingsmiddel, des te groter de aantrekkingskracht en de motivatie om te eten. Onderzoekers adopteerden de verkorte term food reward (reward = beloning) om naar deze vorm van eetgedrag en onderliggende processen te verwijzen (Kelly \& Berridge, 2002; Wise, 2006). Helaas bleef kwalitatief onderzoek naar food reward lange tijd moeilijk vanwege zijn subjectieve aard en geschiedde voornamelijk aan de hand van zelfrapportages ("Hoe graag wilt u dit eten?" of "Hoe lekker vindt u dit?"). Dit probleem veranderde met de komst van neuroimaging onderzoeksmethoden, zoals functionele magnetische resonantie imaging (fMRI). Met behulp van fMRI kunnen onderzoekers activiteit meten in de hersengebieden die betrokken zijn bij de verwerking van food reward (ie., mesocorticolimbische circuit). Met behulp van deze methoden hebben onderzoekers in de afgelopen jaren steeds meer en beter inzicht gekregen in factoren die food reward processen beïnvloeden en daarmee dus ook het eetgedrag aansturen.

Hoofdstuk 1 geeft een historisch overzicht van food reward onderzoek. Er wordt beschreven hoe eetgedragonderzoekers in de loop der jaren hun aandacht verschoven van een voornamelijk homeostatisch perspectief, naar een kijk op eetgedrag welk ook food reward processen omvatte. Vervolgens wordt de 
incentive motivation theorie besproken en het begrip food reward gedefinieerd (Berridge, 2004; Bindra, 1978; Bolles, 1972; Toates, 1986). FMRI is de voornaamste onderzoeksmethode in de studies besproken in dit proefschrift en daarom wordt het verhaal over de incentive motivation theorie gevolgd door een korte uitleg over fMRI en zijn toepassing in food reward onderzoek. Het onderzoek naar food reward heeft ook theorieën met betrekking tot verstoord eetgedrag geïnspireerd, bijvoorbeeld met betrekking tot Anorexia Nervosa. In een kort overzicht worden theorieën over abnormaal eetgedrag beschreven. Als laatste worden de doelen van het huidige proefschrift gepresenteerd, zijnde: met behulp van fMRI, onderzoek doen naar de invloed van calorische dichtheid, honger, aandacht en cognitieve strategieën op mesocorticolimbische activiteit en verstoringen in food reward processen bij Anorexia Nervosa patiënten.

In hoofdstuk 2 wordt een fMRI studie gepresenteerd waarbij we onderzoek deden naar de effecten van calorische inhoud, voedsel deprivatie en aandacht op food reward processen bij gezonde vrouwen. Het idee om de invloed van calorische dichtheid op food reward processen te onderzoeken ontstond vanuit de optimal foraging theory (MacArthur \& Pianka, 1966). Deze theorie veronderstelt dat organismen op een dusdanige manier voedsel zoeken, dat hun energie-inname wordt gemaximaliseerd. Dit zou, evolutionair gezien, voordelen hebben omdat het de overlevingskans van het organisme vergroot. Inderdaad, verschillende onderzoeken tonen aan dat mensen zich gemakkelijker overeten met hoogcalorische voeding in vergelijking met laagcalorische voeding. Het is echter moeilijk om alleen de effecten van calorische dichtheid op food reward processen te onderzoeken, omdat calorische dichtheid moeilijk te onderscheiden is van smakelijkheid. Immers, de twee gaan vaak hand in hand: hoe hoger de calorische inhoud, hoe smakelijker de voeding (Green, Wales, Lawton \& Blundell, 2000). Daarom was het doel van deze eerste studie om de invloed van een hoog- versus laagcalorische dichtheid op food reward processen te bestuderen, terwijl de smakelijkheid tussen hoog- en laagcalorische voeding gelijk wordt gehouden. Het tweede doel was om de effecten van voedseldeprivatie op food reward processen te bestuderen. Ook al veronderstelt de incentive motivation theorie dat homeostatische processen niet voldoende zijn om eetgedrag te sturen, ze hebben wel degelijk invloed op de aantrekkingskracht van voedsel. Dit fenomeen wordt heel duidelijk omschreven door het gezegde "Honger maakt rauwe bonen zoet". Bovendien lijken de 
effecten van honger op food reward processen nogal specifiek te zijn: onderzoek toont aan dat wanneer men zijn voedselinname beperkt (bijvoorbeeld tijdens een dieet), voornamelijk de verlangens naar hoogcalorische voeding worden vergroot (Gilhooly et al., 2007). Het derde en laatste doel van dit onderzoek was om uit te zoeken of er een functioneel onderscheid tussen de food reward processen in de amygdala en die van de orbitofrontale cortex gemaakt kon worden. Hoewel beide structuren in verband worden gebracht met de verwerking van de relatie tussen een voedingsmiddel en de daarbij behorende beloning (Baxter \& Murray, 2002; Kringelbach, 2005), lijkt het gezien de efficiëntie van ons brein zeer onwaarschijnlijk dat beide structuren exact dezelfde taak uitvoeren. Onderzoek uit het verleden leek te suggereren dat een functioneel onderscheid tussen amygdala en orbitofrontale cortex gemaakt kan worden aan de hand van de mate van aandacht nodig bij de verwerking van de voedingsmiddel - beloning associaties: de amygdala zou deze automatisch verwerken, terwijl in de orbitofrontale cortex een meer bewuste verwerking plaatsvindt. Om deze veronderstellingen te toetsen, werden foto's van hoog- en laagcalorische voedingsmiddelen (hypothese 1) aan gezonde vrouwen getoond, nadat zij een voedseldeprivatie van 18 uur hadden ondergaan, of nadat zij een verzadigde lunch hadden gegeten (hypothese 2). Bovendien werd de mate van bewuste verwerking van de voedingsplaatjes gemanipuleerd door de aandacht van de vrouwen te richten op of de voeding of een neutraal aspect van hetzelfde voedingsplaatje (hypothese 3). FMRI resultaten lieten zien dat de verzadigde vrouwen een verhoogde mesocorticolimbische activiteit hadden bij het zien van laagcalorische voeding en een verhoogde activiteit in diezelfde hersenstructuren bij het zien van hoogcalorische voeding wanneer zij 18 uur niet hadden gegeten. Deze bevinding toont aan dat calorische dichtheid inderdaad food reward processen beïnvloedt en dat de mate van invloed samenhangt met de mate van voedseldeprivatie. Verdere resultaten lieten zien dat de aandachtmanipulatie een grote invloed had op activiteit in zowel de amygdala als de orbitofrontale cortex; de vrouwen vertoonden alleen activiteit in beide structuren wanneer de deelnemers bewust het voedsel evalueerden, maar niet wanneer zij hun aandacht richtte op een neutraal aspect. Deze bevindingen toonden aan dat er geen functioneel onderscheid gemaakt kan worden tussen de amygdala en de orbitofrontale cortex op basis van verschillen in aandacht. Deze resultaten toonden wel aan dat vervolgonderzoek naar de invloed van aandacht op food 
reward processen wel eens bijzonder informatief zou kunnen zijn.

Anorexia Nervosa patiënten hongeren zichzelf uit in een wereld waarin er sprake is van een overmaat aan voedsel. Dit is erg bijzonder, vooral omdat veel mensen juist moeite lijken te hebben met het beperken van hun voedselinname. Hoe komt het dat Anorexia Nervosa patiënten zo succesvol zijn in niet eten? De studie beschreven in hoofdstuk 3 onderzocht deze vraag door mogelijke verstoringen in food reward processen te onderzoeken, terwijl Anorexia Nervosa patiënten naar hoog- en laagcalorische voedselplaatjes keken en de smakelijkheid, geur, textuur en smaak van de getoonde voedingsmiddelen probeerden voor te stellen. Er werd verwacht dat, hoewel Anorexia Nervosa patiënten grote homeostatische tekorten hebben, hun mesocorticolimbische activiteit verminderd zou zijn en vergelijkbaar met dat van verzadigde gezonde vrouwen (hypothese 1). Net als in de vorige studie werd ook aandacht gemanipuleerd. Met deze manipulatie werd onderzocht of food reward processen bij Anorexia Nervosa patiënten wel normaal verlopen wanneer zij niet bewust met voeding bezig zijn, maar moeten letten op een neutraal aspect (hypothese 2). Tot slot, anemie (i.e., ijzertekort) is een veel voorkomende aandoening bij Anorexia Nervosa patiënten (Cleary, Gaudiani, \& mehler, 2010), welk resulteert in verlaagde hemoglobine niveaus in hun bloed. Omdat de sterkte van het fMRI signaal afhankelijk is van de hoeveelheid hemoglobine in het bloed, zou een verlaging in hemoglobine een mogelijk verstorende factor kunnen zijn in het onderzoek naar een mogelijke verlaging in food reward activiteit bij Anorexia Nervosa patiënten. Om deze mogelijke verstoringen te onderzoeken werden twee neutrale condities toegevoegd. In deze conditie werden neutrale voorwerpen getoond. Er werd verondersteld dat deze neutrale condities niet zouden mogen resulteren in verschillen in hersenactiviteit tussen Anorexia Nervosa patiënten en gezonde vrouwen (hypothese 3). De fMRI resultaten lieten zien dat de evaluatie van hoog- en laagcalorische voeding leidde tot verhoogde mesocorticolimbische activiteit in zowel de hongerige als de verzadigde gezonde vrouwen, maar niet in de Anorexia Nervosa patiënten. Deze resultaten laten zien dat food reward activiteit verminderd is bij Anorexia Nervosa patiënten, zelfs in vergelijking met de verzadigde vrouwen. Deze bevinding kon niet worden toegeschreven aan een mogelijke invloed van anemie, omdat de neutrale condities tot vergelijkbare of zelfs verhoogde activiteit leidde bij de Anorexia Nervosa patiënten in vergelijking met de gezonde vrouwen. Een zeer interessante 
bevinding was dat food reward activiteit, vergelijkbaar met die van hongerige gezonde vrouwen, wel waarneembaar was wanneer de patiënten moesten letten op een neutraal aspect in plaats van hoogcalorische voeding. Deze activiteit ging samen met een verhoogde activiteit in de rechter dorsolaterale prefrontale cortex en de linker anterieure temporale cortex, structuren waarvan de activiteit in eerder onderzoek werd gerelateerd aan zelfcontrole (Hare, 2009). Deze laatste bevinding toont aan dat Anorexia Nervosa patiënten wel reageren op hoogcalorische voedselverleidingen, maar dat deze reactie gepaard gaat met een verhoogde zelfcontrole. Deze bevindingen laten zien waarom niet eten relatief gemakkelijk is in Anorexia nervosa patiënten: wanneer zij aandacht besteden aan voedsel dan is de food reward activiteit verlaagd en is er geen verhoogde zelfcontrole nodig. Wanneer echter hoogcalorische voeding wordt gepresenteerd en de aandacht wordt gericht op een neutraal aspect dan is er wel food reward activiteit. Dit betekent dat Anorexia Nervosa patiënten wel nog reageren op voedselverleidingen, maar wanneer het food reward circuit reageert op deze verleiding, dan treedt er gelijktijdig een verhoging op in zelfcontrole gebieden. Deze combinatie van verhoogde food reward en verhoogde zelfcontrole zorgt ervoor dat Anorexia Nervosa patiënten gemakkelijk aan de verleidingen van smakelijke voeding kunnen weerstaan.

Veel populaire diëten beweren dat je de onweerstaanbare verleiding van zeer smakelijke voeding kunt weerstaan, door de manier waarop je over voeding denkt actief te beheersen en te veranderen. De studie beschreven in hoofdstuk 4 onderzocht de invloed van drie verschillende manieren van cognitieve beheersingsstrategieën op mesocorticolimbische activiteit en op zelfgerapporteerde voedselverlangens. Deze cognitieve beheersingsstrategieën waren: (1) verhoging, waarbij de intensiteit van innerlijke reacties worden versterkt (Gross, 2006), (2) cognitieve herwaardering, waarbij de manier waarop over het voedingsmiddel wordt gedacht zodanig verandert dat ook de impact daarvan verandert (Gross, 2006), en (3) onderdrukking, waarbij gedachten of reacties met betrekking tot voedselverlangens actief worden onderdrukt (Wenzlaff \& Wegner, 2000; Gross, 2006). Onderdrukking is een cognitieve beheersingsstrategie die een verhoogde mate van zelfcontrole vereist gedurende de gehele toepassing van deze techniek. In tegenstelling, cognitieve herwaardering brengt veranderingen teweeg vroeg in het verwerkingsproces, waardoor deze strategie gemakkelijker gedurende een langere tijd volgehouden kan worden (Gross \& John, 2003). Er 
werd dan ook verwacht dat cognitieve herwaardering het meest succesvol zou zijn in het verminderen van mesocorticolimbische activiteit en zelfgerapporteerde voedselverlangens. De zelfrapportage resultaten toonden aan dat verhoging, voedselverlangens versterkte in vergelijking met de andere twee strategieën en dat er geen verschil was in voedselverlangens tussen de onderdrukking en cognitieve herwaarderingstrategie. FMRI resultaten lieten een verhoogde mesocorticolimbische activiteit zien na verhoging. In tegenstelling tot de verwachtingen bleek onderdrukking meer effectief in het verminderen van activiteit in zeer belangrijke mesocorticolimbische gebieden (i.e., ventrale tegmentale gebied en het ventrale striatum) dan cognitieve herwaardering. Echter, de resultaten lieten ook zien dat onderdrukking gepaard ging met een grotere toename in prefrontale activiteit in vergelijking met cognitieve herwaardering. Dit betekent dat onderdrukking een grotere mate van cognitieve activiteit vereiste, welke in overeenstemming was met de verwachtingen. Samengevat, huidige resultaten ondersteunen de veronderstelling dat mesocorticolimbische activiteit beïnvloedt wordt door cognitieve beheersingsstrategieën. Deze bevinding kan voornamelijk relevant zijn voor de ontwikkeling van succesvolle behandelingen van verstoord eetgedrag.

Hoofdstuk 5 is een bespreking van de voornaamste theorieën over cognitieve biasen en verstoringen in cognitieve processen bij personen met een eetstoornis. In dit hoofdstuk wordt bediscussieerd hoe en of de beïnvloeding van cognitieve biasen in eetstoornispatiënten gepaard gaat met veranderingen in eetstoornissymptomen. Sommige van deze ideeën werden direct geïnspireerd door het fMRI onderzoek besproken in dit proefschrift. Dit hoofdstuk illustreert hoe fMRI onderzoek bijdraagt aan een vergroting van het begrip van eetstoornissen en de ontwikkeling van mogelijke toekomstige behandelingen.

In hoofdstuk 6 worden de bevindingen van de in dit proefschrift gepresenteerde onderzoeken samengevat en implicaties voor toekomstig onderzoek besproken. Dit proefschrift laat zien dat fMRI onderzoek naar food reward processen tot belangrijke inzichten leidt in factoren die (ab)normaal eetgedrag beïnvloeden. Zo werd bijvoorbeeld aangetoond dat voedseldeprivatie hoogcalorische voeding verleidelijker maakt. Deze bevinding heeft belangrijke implicaties voor diegenen die hun lichaamsgewicht proberen te verminderen. Bovendien, fMRI resultaten tonen aan dat alleen de bewuste evaluatie van voeding leidt tot een verhoging in mesocorticolimbische activiteit. Deze bevinding heeft vervolgens geleid tot een 
onderzoek naar de invloed van cognitieve beheersingsstrategieën op food reward processen. Inderdaad, huidige resultaten tonen aan dat gezonde vrouwen mesocorticolimbische activiteit kunnen beïnvloeden door middel van verhoging, onderdrukking en cognitieve herwaardering. Deze laatste bevinding is vooral relevant wanneer wij kijken naar de bevinding van verlaagde food reward activiteit in Anorexia Nervosa patiënten en al eerder aangetoonde verhoogde food reward activiteit in personen met overgewicht (Kaye et al., 2009; Rothermund et al., 2007; Stoeckel, 2008). Toekomstig onderzoek is dan ook noodzakelijk om te toetsen of het trainen van patiënten in het veranderen van mesocorticolimbische activiteit door middel van cognitieve beheersingsstrategieën (bijvoorbeeld met behulp van geavanceerde technieken zoals realtime fMRI) leidt tot langdurige veranderingen in mesocorticolimbische food reward activiteit en daaraan gerelateerd eetgedrag. 
References 


\section{REFERENCES}

Alcaro, A., Huber, R., \& Panksepp, J. (2007). Behavioral functions of the mesolimbic dopaminergic system: an affective neuroethological perspective. Brain Research Reviews, 56, 283-321.

Allan, K., \& Rugg, M. D. (1997). An event-related potential study of explicit memory on tests of cued recall and recognition. Neuropsychologia, 35, 387-397.

Amodio, D. M., \& Frith, C. D. (2006). Meeting of minds: the medial frontal cortex and social cognition. Nature Reviews Neuroscience, 7, 268-277.

Anand, B. K., \& Brobeck, J. R. (1951). Hypothalamic control of food intake in rats and cats. The Yale Journal of Biology and Medicine, 24, 123-140.

APA. (2000). Diagnostic and Statistical Manual of Mental Disorders, Fourth Edition, Text Revision. Washington DC: American Psychiatric Association.

Appelhans, B. M. (2009). Neurobehavioral inhibition of reward-driven feeding: implications for dieting and obesity. Obesity „, 17, 640-647.

Arana, F. S., Parkinson, J. A., Hinton, E., Holland, A. J., Owen, A. M., \& Roberts, A. C. (2003). Dissociable contributions of the human amygdala and orbitofrontal cortex to incentive motivation and goal selection. Journal of Neuroscience, 23, 9632-9638.

Assanand, S., Pinel, J. P., \& Lehman, D. R. (1998). Teaching Theories of Hunger and Eating: Overcoming Students' Misconceptions. Teaching of Psychology, 25, 44-46.

Astrup, A., Greenway, F. L., Ling, W., Pedicone, L., Lachowicz, J., Strader, C. D., et al. (2007). Randomized controlled trials of the D1/D5 antagonist ecopipam for weight loss in obese subjects. Obesity, 15, 1717-1731.

Augustine, J. R. (1996). Circuitry and functional aspects of the insular lobe in primates including humans. Brain Research. Brain Research Reviews, 22, 229-244.

Barsh, G. S., \& Schwartz, M. W. (2002). Genetic approaches to studying energy balance: perception and integration. Nature Reviews Genetics, 3, 589-600.

Baxter, M. G., \& Murray, E. A. (2002). The amygdala and reward. Nature Reviews Neuroscience, 3, 563-573.

Beck, A. T. (1976). Cognitive therapy and the emotional disorders. New York: International Universities Press.

Beck., J. (2007). The Beck diet solution. lowa: Oxmoor House.

Berridge, K. C. (1996). Food reward: brain substrates of wanting and liking. Neuroscience and Biobehavioral Reviews, 20, 1-25. 
Berridge, K. C. (2004). Motivation concepts in behavioral neuroscience. Physiology and Behavior, 81, 179-209.

Berridge, K. C., \& Kringelbach, M. L. (2008). Affective neuroscience of pleasure: reward in humans and animals. Psychopharmacology, 199, 457-480.

Bindra, D. (1978). How adaptive behavior is produced: a perceptual-motivation alternative to response reinforcement. Behaviour and Brain Sciences, 79, 41-91.

Bishop, S. J., Duncan, J., \& Lawrence, A. D. (2004). State anxiety modulation of the amygdala response to unattended threat-related stimuli. Journal of Neuroscience, 24, 10364-10368.

Bluher, S., \& Mantzoros, C. S. (2009). Leptin in humans: lessons from translational research. The American Journal of Clinical Nutrition, 89, 991S-997S.

Boeka, A. G., \& Lokken, K. L. (2006). The lowa gambling task as a measure of decision making in women with bulimia nervosa. Journal of the International Neuropsychological Society, 12, 741-745.

Bolles, R. C. (1972). Reinforcement, expectancy, and learning. Psychology Review, 79, 394-409.

Bolles, R. C. (1980). Some functionalistic thoughts about regulation. In: Toates TW, Halliday TW, editors. Analysis of motivational processes. New York: Academic Press.

Bonson, K. R., Grant, S. J., Contoreggi, C. S., Links, J. M., Metcalfe, J., Weyl, H. L., et al. (2002). Neural systems and cue-induced cocaine craving. Neuropsychopharmacology, 26, 376-386.

Booth, D. A. (1991). Learned ingestive motivation and the pleasures of the palate. In: Bolles RC, editor. The hedonics of taste. Hillsdale (NJ): Lawrence Erlbaum Associates.

Brand, M., Franke-Sievert, C., Jacoby, G. E., Markowitsch, H. J., \& Tuschen-Caffier, B. (2007). Neuropsychological correlates of decision making in patients with bulimia nervosa. Neuropsychology, 21, 742-750.

Bridgman, P. W. (1982). Einstein's theories and the operational point of view. La Salle: Cambridge University Press.

Brown, P. L., \& Jenkins, H. M. (1968). Auto-shaping of the pigeon's key-peck. Journal of the Experimental Analysis of Behavior, 11, 1-8.

Brownell, K. D. (2005). The chronicling of obesity: growing awareness of its social, economic, and political contexts. Journal of Health Politics, Policy and Law, 30, 955964. 
Bryant, M., Truesdale, K. P., \& Dye, L. (2006). Modest changes in dietary intake across the menstrual cycle: implications for food intake research. The British Journal of Nutrition, 96, 888-894.

Cabanac, M. (1979). Sensory Pleasure. Quarterly Review Biology, 54, 1-29.

Caine, S. B., \& Koob, G. F. (1994). Effects of mesolimbic dopamine depletion on responding maintained by cocaine and food. J Exp Anal Behav, 61, 213-221.

Cannon, W. B. (1932). Wisdom of the body. New York: Norton.

Carter, C. S., Braver, T. S., Barch, D. M., Botvinick, M. M., Noll, D., \& Cohen, J. D. (1998). Anterior cingulate cortex, error detection, and the online monitoring of performance. Science, 280, 747-749.

Carver, C. S., \& White, T. L. (1994). Behavioral inhibition, behavioral activation, and affective responses to impending reward and punishment: the BIS/BAS Scales. Journal of Personality and Social Psychology, 67, 319-333.

Cavedini, P., Bassi, T., Ubbiali, A., Casolari, A., Giordani, S., Zorzi, C., et al. (2004). Neuropsychological investigation of decision-making in anorexia nervosa. Psychiatry Research, 127, 259-266.

Cleary, B. S., Gaudiani, J. L., \& Mehler, P. S. (2010). Interpreting the complete blood count in anorexia nervosa. Eating Disorders, 18, 132-139.

Clifton, P. M., \& Keogh, J. (2007). Metabolic effects of high-protein diets. Current Atherosclerosis Reports, 9, 472-478.

Cooper, M. (1997). Bias in interpretation of ambiguous scenarios in eating disorders. Behaviour Research and Therapy, 35, 619-626.

Craig, A. D. (2002). How do you feel? Interoception: the sense of the physiological condition of the body. Nature Reviews Neuroscience, 3, 655-666.

Craig, A. D. (2003). Interoception: the sense of the physiological condition of the body. Current Opinion in Neurobiology, 13, 500-505.

Craig, A. D. (2009). How do you feel--now? The anterior insula and human awareness. Nature Reviews Neuroscience, 10, 59-70.

Currin, L., Schmidt, U., Treasure, J., \& Jick, H. (2005). Time trends in eating disorder incidence. The British Journal of Psychiatry, 186, 132-135.

D'Ardenne, K., McClure, S. M., Nystrom, L. E., \& Cohen, J. D. (2008). BOLD responses reflecting dopaminergic signals in the human ventral tegmental area. Science, 319, 1264-1267. 
Davis, C., \& Carter, J. C. (2009). Compulsive overeating as an addiction disorder. A review of theory and evidence. Appetite, 53, 1-8.

Davis, C., Patte, K., Levitan, R., Reid, C., Tweed, S., \& Curtis, C. (2007). From motivation to behaviour: a model of reward sensitivity, overeating, and food preferences in the risk profile for obesity. Appetite, 48, 12-19.

Davy, S. R., Benes, B. A., \& Driskell, J. A. (2006). Sex differences in dieting trends, eating habits, and nutrition beliefs of a group of midwestern college students. Journal of the American Dietetic Association, 106, 1673-1677.

De Araujo, I. E., \& Rolls, E. T. (2004). Representation in the human brain of food texture and oral fat. The Journal of Neuroscience, 24, 3086-3093.

De Araujo, I. E., Rolls, E. T., Velazco, M. I., Margot, C., \& Cayeux, I. (2005). Cognitive modulation of olfactory processing. Neuron, 46, 671-679.

De Ruiter, C., \& Brosschot, J. F. (1994). The emotional Stroop interference effect in anxiety: attentional bias or cognitive avoidance? Behaviour Research and Therapy, $32,315-319$.

De Zubicaray, G., McMahon, K., Eastburn, M., Pringle, A., \& Lorenz, L. (2006). Classic identity negative priming involves accessing semantic representations in the left anterior temporal cortex. Neuroimage, 33, 383-390.

DeCharms, R. C. (2008). Applications of real-time fMRI. Nature Reviews Neuroscience, 9, 720-729.

Deichmann, R. (2005). Optimized RF excitation for anatomical brain imaging of the occipital lobe using the 3D MDEFT sequence and a surface transmit coil. Magnetic Resonance in Medicine, 53, 1212-1216.

Deichmann, R., Gottfried, J. A., Hutton, C., \& Turner, R. (2003). Optimized EPI for fMRI studies of the orbitofrontal cortex. Neuroimage, 19, 430-441.

Delparigi, A., Chen, K., Salbe, A. D., Hill, J. O., Wing, R. R., Reiman, E. M., et al. (2007). Successful dieters have increased neural activity in cortical areas involved in the control of behavior. International Journal of Obesety, 31, 440-448.

Dobson, K. S., \& Dozois, D. J. (2004). Attentional biases in eating disorders: a metaanalytic review of Stroop performance. Clinical Psychological Review, 23, 10011022.

Drewnowski, A., Maillot, M., \& Darmon, N. (2008). Testing nutrient profile models in relation to energy density and energy cost. European Journal of Clinical Nutrition, 63, 674-683.

Duzel, E., Bunzeck, N., Guitart-Masip, M., Wittmann, B., Schott, B. H., \& Tobler, P. N. (2009). Functional imaging of the human dopaminergic midbrain. Trends in Neuroscience, 32, 321-328. 
Epstein, L. H., Leddy, J. J., Temple, J. L., \& Faith, M. S. (2007). Food reinforcement and eating: a multilevel analysis. Psychological Bulletin, 133, 884-906.

Epstein, L. H., Myers, M. D., Raynor, H. A., \& Saelens, B. E. (1998). Treatment of pediatric obesity. Pediatrics, 101, 554-570.

Fairburn, C. G. (2008). Eating disorders: the transdiagnostic view and the cognitive behavioral theory. New York: The Guilford Press.

Faunce, G. J., \& Job, R. F. (2000). The Stroop colour-naming task and addictive behaviour: some recommendations. Addiction, 95, 1438-1442.

Field, M., Duka, T., Eastwood, B., Child, R., Santarcangelo, M., \& Gayton, M. (2007). Experimental manipulation of attentional biases in heavy drinkers: do the effects generalise? Psychopharmacology, 192, 593-608.

Field, M., Mogg, K., \& Bradley, B. P. (2005). Craving and cognitive biases for alcohol cues in social drinkers. Alcohol Alcohol, 40, 504-510.

Fox, E., Russo, R., Bowles, R., \& Dutton, K. (2001). Do threatening stimuli draw or hold visual attention in subclinical anxiety? Journal of Experimental Psychology. General, $130,681-700$.

Francis, J. A., Stewart, S. H., \& Hounsell, S. (1997). Dietary restraint and the selective processing of forbidden and non-forbidden food words. Cognitive Therapy and Research., 21, 633-646.

Franken, I. H., Kroon, L. Y., \& Hendriks, V. M. (2000). Influence of individual differences in craving and obsessive cocaine thoughts on attentional processes in cocaine abuse patients. Addictive Behaviors, 25, 99-102.

Frankenburg, F. R. (1984). Hoarding in anorexia nervosa. The British Journal of Medical Psychology, 57, 57-60.

Freeman, R., Touyz, S., Sara, G., Rennie, C., Gordon, E., \& Beumont, P. (1991). In the eye of the beholder: Processing body shape information in anorexic and bulimic patients. International Journal of Eating Disorders 10, 709-714.

Friedman, M. I., Ulrich, P., \& Mattes, R. D. (1999). A figurative measure of subjective hunger sensations. Appetite, 32, 395-404.

Geday, J., Gjedde, A., Boldsen, A. S., \& Kupers, R. (2003). Emotional valence modulates activity in the posterior fusiform gyrus and inferior medial prefrontal cortex in social perception. Neuroimage, 18, 675-684.

Ghashghaei, H. T., \& Barbas, H. (2002). Pathways for emotion: interactions of prefrontal and anterior temporal pathways in the amygdala of the rhesus monkey. Neuroscience, 115, 1261-1279. 
Gilbert, S. J., Frith, C. D., \& Burgess, P. W. (2005). Involvement of rostral prefrontal cortex in selection between stimulus-oriented and stimulus-independent thought. European Journal of Neuroscience, 21, 1423-1431.

Gilhooly, C. H., Das, S. K., Golden, J. K., McCrory, M. A., Dallal, G. E., Saltzman, E., et al. (2007). Food cravings and energy regulation: the characteristics of craved foods and their relationship with eating behaviors and weight change during 6 months of dietary energy restriction. International Journal of Obesity, 31, 1849-1858.

Glauert, R., Rhodes, G., Fink, B., \& Grammer, K. (2010). Body dissatisfaction and attentional bias to thin bodies. The International Journal of Eating Disorders, 43, 4249.

Goldin, P. R., McRae, K., Ramel, W., \& Gross, J. J. (2008). The neural bases of emotion regulation: reappraisal and suppression of negative emotion. Biological Psychiatry, $63,577-586$

Goldstein, R. Z., \& Volkow, N. D. (2002). Drug addiction and its underlying neurobiological basis: neuroimaging evidence for the involvement of the frontal cortex. American Journal of Psychiatry, 159, 1642-1652.

Goldzieher, J. W. (1994). Are low-dose oral contraceptives safer and better? American Journal of Obstetrics and Gynecology, 171, 587-590.

Gottfried, J. A., O'Doherty, J., \& Dolan, R. J. (2003). Encoding predictive reward value in human amygdala and orbitofrontal cortex. Science, 301, 1104-1107.

Grabenhorst, F., Rolls, E. T., \& Bilderbeck, A. (2008). How cognition modulates affective responses to taste and flavor: top-down influences on the orbitofrontal and pregenual cingulate cortices. Cerebral Cortex, 18, 1549-1559.

Green, S. M., Wales, J. K., Lawton, C. L., \& Blundell, J. E. (2000). Comparison of high-fat and high-carbohydrate foods in a meal or snack on short-term fat and energy intakes in obese women. The British Journal of Nutrition, 84, 521-530.

Grill-Spector, K., \& Malach, R. (2001). fMR-adaptation: a tool for studying the functional properties of human cortical neurons. Acta Psychologica, 107, 293-321.

Gross, J. J. (2006). Handbook of emotion regulation. East Sussex: The Guilfort Press.

Gross, J. J., \& John, O. P. (2003). Individual differences in two emotion regulation processes: implications for affect, relationships, and well-being. Journal of Personality and Social Psychology, 85, 348-362.

Hahn, T. M., Breininger, J. F., Baskin, D. G., \& Schwartz, M. W. (1998). Coexpression of Agrp and NPY in fasting-activated hypothalamic neurons. Nature Neuroscience, 1, 271-272.

Hansen, C. H., \& Hansen, R. D. (1988). Finding the face in the crowd: an anger superiority effect. Journal of Personality and Social Psychology, 54, 917-924. 
Happe, F., \& Frith, U. (2006). The weak coherence account: detail-focused cognitive style in autism spectrum disorders. Journal of Autism and Developmental Disorders, 36, 525 .

Hare, T. A., Camerer, C. F., \& Rangel, A. (2009). Self-control in decision-making involves modulation of the vmPFC valuation system. Science, 324, 646-648.

Hargreaves, D., \& Tiggemann, M. (2002). The effect of television commercials on mood and body dissatisfaction: the role of appaerance-schema activation. Journal of Social and Clinical Psychology, 21, 287-308.

Harrison, A., Sullivan, S., Tchanturia, K., \& Treasure, J. (2009). Emotion recognition and regulation in anorexia nervosa. Clinical Psychology \& Psychotherapy, 16, 348-356.

Hecht, D. (2010). Transcranial direct current stimulation in the treatment of anorexia. Medical Hypotheses., 74, 1044-1047.

Henderson, J. M., Pollatsek, A., \& Rayner, K. (1989). Covert visual attention and extrafoveal information use during object identification. Perception \& Psychophysics, 45, 196-208.

Henson, R. (2005). What can functional neuroimaging tell the experimental psychologist? The Quarterly Journal of Experimental Psychology, 58, 193-233.

Herman, C. P., Polivy, J., Pliner, P., Threlkeld, J., \& Munic, D. (1978). Distractibility in dieters and nondieters: an alternative view of "externality". Journal of Personality and Social Psychology, 36, 536-548.

Hermans, D., Pieters, G., \& Eelen, P. (1998). Implicit and explicit memory for shape, body weight, and food-related words in patients with anorexia nervosa and nondieting controls. Journal of Abnormal Psychology, 107, 193-202.

Heymsfield, S. B., Greenberg, A. S., Fujioka, K., Dixon, R. M., Kushner, R., Hunt, T., et al. (1999). Recombinant leptin for weight loss in obese and lean adults: a randomized, controlled, dose-escalation trial. JAMA, 282, 1568-1575.

Holliday, J., Tchanturia, K., Landau, S., Collier, D., \& Treasure, J. (2005). Is impaired setshifting an endophenotype of anorexia nervosa? American Journal of Psychiatry, 162, 2269-2275.

Hu, J., Zhu, C., \& Huang, M. (2009). The endocannabinoid system: a new pharmacological target for obesity treatment? Neuroscience Bulletin, 25, 153-160.

Huettel, S. A., Song, A. W., \& McCarthy, G. (2004). Functional Magnetic Resonance Imaging. Massachusetts: Sinauer Associates Inc.

Hunt, J., \& Cooper, M. (2001). Selective memory bias in women with bulimia nervosa and women with depression. Behavioural and Cognitive Psychotherapy 29, 93-102. 
Iaria, G., Fox, C. J., Waite, C. T., Aharon, I., \& Barton, J. J. (2008). The contribution of the fusiform gyrus and superior temporal sulcus in processing facial attractiveness: neuropsychological and neuroimaging evidence. Neuroscience, 155, 409-422.

Ikemoto, S. (2007). Dopamine reward circuitry: two projection systems from the ventral midbrain to the nucleus accumbens-olfactory tubercle complex. Brain Research Reviews, 56, 27-78.

Jacoby, L. L., Allan, L. G., Collins, J. C., \& Larwill, L. K. (1988). Memory influences subjective experience: Noise judgment. Journal of Experimental Psychology: Learning, Memory, and Cognition, 14, 240-247.

Jansen, A., Nederkoorn, C., \& Mulkens, S. (2005). Selective visual attention for ugly and beautiful body parts in eating disorders. Behaviour Research and Therapy, 43, 183196.

Jansen, A., Smeets, T., Boon, B., Nederkoorn, C., Roefs, A., \& Mulkens, S. (2007). Vulnerability to interpretation bias in overweight children. Psychology \& Health, 22, 561-574.

Jansen, A., Smeets, T., Martijn, C., \& Nederkoorn, C. (2006). I see what you see: the lack of a self-serving body-image bias in eating disorders. The British Journal of Clinical Psychology, 45, 123-135.

Jeffery, R. W., Drewnowski, A., Epstein, L. H., Stunkard, A. J., Wilson, G. T., Wing, R. R., et al. (2000). Long-term maintenance of weight loss: current status. Health Psychology, 19, 5-16.

Johansson, L., Ghaderi, A., \& Andersson, G. (2005). Stroop interference for food- and body-related words: a meta-analysis. Eating Behaviors, 6, 271-281.

Johnson, J., \& Vickers, Z. (1992). Factors influencing sensory-specific satiety. Appetite, 19, $15-31$.

Kable, J. W., \& Glimcher, P. W. (2007). The neural correlates of subjective value during intertemporal choice. Nature Neuroscience, 10, 1625-1633.

Kaye, W. H., Fudge, J. L., \& Paulus, M. (2009). New insights into symptoms and neurocircuit function of anorexia nervosa. Nature Reviews Neuroscience, 10, 573584 .

Keesey, R. E., \& Powley, T. L. (2008). Body energy homeostasis. Appetite, 51, 442-445.

Kelley, A. E., \& Berridge, K. C. (2002). The neuroscience of natural rewards: relevance to addictive drugs. The Journal of Neuroscience, 22, 3306-3311.

Kensinger, E. A., \& Schacter, D. L. (2006). Processing emotional pictures and words: effects of valence and arousal. Cognitive, Affective \& Behavioural Neuroscience, 6, $110-126$. 
Killgore, W. D., Young, A. D., Femia, L. A., Bogorodzki, P., Rogowska, J., \& YurgelunTodd, D. A. (2003). Cortical and limbic activation during viewing of high- versus low-calorie foods. Neuroimage, 19, 1381-1394.

King, G. A., Polivy, J., \& Herman, C. P. (1991). Cognitive aspects of dietary restraint: Effects on person memory. International Journal of Eating Disorders, 10, 313-321.

Kosten, T. R., Scanley, B. E., Tucker, K. A., Oliveto, A., Prince, C., Sinha, R., et al. (2006). Cue-induced brain activity changes and relapse in cocaine-dependent patients. Neuropsychopharmacology, 31, 644-650.

Kreitzer, A. C. (2009). Physiology and pharmacology of striatal neurons. Annual Review of Neuroscience, 32, 127-147.

Kringelbach, M. L. (2004). Food for thought: hedonic experience beyond homeostasis in the human brain. Neuroscience, 126, 807-819.

Kringelbach, M. L. (2005). The human orbitofrontal cortex: linking reward to hedonic experience. Nature Reviews Neuroscience, 6, 691-702.

Kringelbach, M. L., O'Doherty, J., Rolls, E. T., \& Andrews, C. (2003). Activation of the human orbitofrontal cortex to a liquid food stimulus is correlated with its subjective pleasantness. Cerebral Cortex, 13, 1064-1071.

Kringelbach, M. L., \& Rolls, E. T. (2004). The functional neuroanatomy of the human orbitofrontal cortex: evidence from neuroimaging and neuropsychology. Progress in Neurobiology, 72, 341-372.

Kristensen, P., Judge, M. E., Thim, L., Ribel, U., Christjansen, K. N., Wulff, B. S., et al. (1998). Hypothalamic CART is a new anorectic peptide regulated by leptin. Nature, $393,72-76$.

LaBar, K. S., Gitelman, D. R., Parrish, T. B., Kim, Y. H., Nobre, A. C., \& Mesulam, M. M. (2001). Hunger selectively modulates corticolimbic activation to food stimuli in humans. Behavioral Neuroscience, 115, 493-500.

Lane, R. D. (2008). Neural substrates of implicit and explicit emotional processes: a unifying framework for psychosomatic medicine. Psychosomatic Medicine, 70, 214231.

Lappalainen, R., \& Epstein, L. H. (1990). A behavioral economics analysis of food choice in humans. Appetite, 14, 81-93.

Lee, M., \& Shafran, R. (2004). Information processing biases in eating disorders. Clinical Psychology Review, 24, 215-238.

Lee, M., \& Shafran, R. (2008). Processing biases in eating disorders: the impact of temporal factors. The International Journal of Eating Disorders, 41, 372-375. 
Lepsien, J., \& Nobre, A. C. (2007). Attentional modulation of object representations in working memory. Cerebral Cortex, 17, 2072-2083.

Lopez, C., Tchanturia, K., Stahl, D., \& Treasure, J. (2009). Weak central coherence in eating disorders: a step towards looking for an endophenotype of eating disorders. Journal of Clinical and Experimental Neuropsychology, 31, 117-125.

MacArthur, R. H., \& Pianka, E. (1966). R.H. MacArthur and E. Pianka, An optimal use of a patchy environment. American Naturalist, 100, 603-609.

MacLeod, C., Mathews, A., \& Tata, P. (1986). Attentional bias in emotional disorders. Journal of Abnormal Psychology, 95, 15-20.

Maren, S. (2008). Pavlovian fear conditioning as a behavioral assay for hippocampus and amygdala function: cautions and caveats. The European Journal of Neuroscience, 28, 1661-1666.

Mathews, A., \& MacLeod, C. (2005). Cognitive vulnerability to emotional disorders. Annual Review of Clinical Psychology, 1, 167-195.

Mesulam, M. M., \& Mufson, E. J. (1982a). Insula of the old world monkey. I. Architectonics in the insulo-orbito-temporal component of the paralimbic brain. The Journal of Comparative Neurology, 21, 1-22.

Mesulam, M. M., \& Mufson, E. J. (1982b). Insula of the old world monkey. III: Efferent cortical output and comments on function. The Journal of Comparative Neurology, $212,38-52$.

Mogg, K., Bradley, B. P., Dixon, C., Fisher, S., Twelftree, H., \& McWilliams, A. (2000). Trait anxiety, defensiveness, and selective processing of treat: An investigation using two measures of attentional bias. Personality \& Individual Differences, 28, 10631077.

Mogg, K., Bradley, B. P., Hyare, H., \& Lee, S. (1998). Selective attention to food-related stimuli in hunger: are attentional biases specific to emotional and psychopathological states, or are they also found in normal drive states? Behaviour Research and Therapy, 36, 227-237.

Mogg, K., Field, M., \& Bradley, B. P. (2005). Attentional and approach biases for smoking cues in smokers: an investigation of competing theoretical views of addiction. Psychopharmacology, 180, 333-341.

Moreno, S., Rodriguez, S., Fernandez, M. C., Tamez, J., \& Cepeda-Benito, A. (2008). Clinical validation of the trait and state versions of the Food Craving Questionnaire. Assessment, 15, 375-387.

Morrison, T., Waller, G., \& Lawson, R. (2006). Attributional style in the eating disorders. The Journal of Nervous and Mental Disease, 194, 303-305. 
Morse, K. L., \& Driskell, J. A. (2009). Observed sex differences in fast-food consumption and nutrition self-assessments and beliefs of college students. Nutrition Research, 29, 173-179.

Mufson, E. J., \& Mesulam, M. M. (1982). Insula of the old world monkey. II: Afferent cortical input and comments on the claustrum. The Journal of Comparative Neurology, 212, 23-37.

Mulkens, S., \& Jansen, A. (2009). Mirror gazing increases attractiveness in satisfied, but not in dissatisfied women: a model for body dysmorphic disorder? Journal of Behavior Therapy and Experimental Psychiatry, 40, 211-218.

Murphy, K. G., Dhillo, W. S., \& Bloom, S. R. (2006). Gut peptides in the regulation of food intake and energy homeostasis. Endocrine Reviews, 27, 719-727.

Murray, E. A. (2007). The amygdala, reward and emotion. Trends in Cognitive Sciences, $11,489-497$.

Murray, E. A., \& Izquierdo, A. (2007). Orbitofrontal cortex and amygdala contributions to affect and action in primates. Annals of the New York Academy of Sciences, 1121, 273-296.

Naqvi, N. H., Rudrauf, D., Damasio, H., \& Bechara, A. (2007). Damage to the insula disrupts addiction to cigarette smoking. Science, 315, 531-534.

Nederkoorn, C., Smulders, F. T., Havermans, R. C., Roefs, A., \& Jansen, A. (2006). Impulsivity in obese women. Appetite, 47, 253-256.

NICE. (2004). Eating disorders-core interventions in the treatment and management of anorexia nervosa, bulimia nervosa and related eating disorders. NICE Clinical Guideline no 9. London: NICE.

O'Doherty, J., Rolls, E. T., Francis, S., Bowtell, R., \& McGlone, F. (2001). Representation of pleasant and aversive taste in the human brain. Journal of Neurophysiology, 85, 1315-1321.

O'Doherty, J., Rolls, E. T., Francis, S., Bowtell, R., McGlone, F., Kobal, G., et al. (2000). Sensory-specific satiety-related olfactory activation of the human orbitofrontal cortex. Neuroreport, 11, 893-897.

Ochsner, K. N., Ray, R. D., Cooper, J. C., Robertson, E. R., Chopra, S., Gabrieli, J. D., et al. (2004). For better or for worse: neural systems supporting the cognitive down- and up-regulation of negative emotion. Neuroimage, 23, 483-499.

Ogawa, S., Tank, D. W., Menon, R., Ellermann, J. M., Kim, S. G., Merkle, H., et al. (1992). Intrinsic signal changes accompanying sensory stimulation: functional brain mapping with magnetic resonance imaging. Proceedings of the National Academy of Sciences of the United States of America, 89, 5951-5955. 
Oh, S. Y., Kim, B. S., \& Choue, R. (2002). Appetite sensations and eating behaviors to complete fasting in obese and non-obese individuals. European Journal of Clinical Nutrition, 56, 86-89.

Oldershaw, A., Hambrook, D., Tchanturia, K., Treasure, J., \& Schmidt, U. (2009). Emotional theory of mind and emotional awareness in recovered anorexia nervosa patients. Psychosomatic Medicine, 72, 73-79.

Olds, J., \& Milner, P. (1954). Positive reinforcement produced by electrical stimulation of septal area and other regions of rat brain. Journal of Comparative and Physiological Psychology, 47, 419-427.

Overbeek, T., Schruers, K., \& Griez, E. (1999). MINI- The Mini International Neuropsychiatric Interview. Dutch version 5.0.0 DSM-IV.

Overduin, J., Jansen, A., \& Louwerse, E. (1995). Stroop interference and food intake. The International Journal of Eating Disorders, 18, 277-285.

Padoa-Schioppa, C., \& Assad, J. A. (2006). Neurons in the orbitofrontal cortex encode economic value. Nature, 441, 223-226.

Paus, T. (2001). Primate anterior cingulate cortex: where motor control, drive and cognition interface. Nature Reviews Neuroscience, 2, 417-424.

Pellegrini, S., Lopez Seal, M. F., \& Papini, M. R. (2008). Scaling relative incentive value: different adjustments to incentive downshift in pigeons and rats. Behavioural Processes, 79, 182-188.

Pessoa, L., Padmala, S., \& Morland, T. (2005). Fate of unattended fearful faces in the amygdala is determined by both attentional resources and cognitive modulation. Neuroimage, 28, 249-255

Pietrowsky, R., Krug, R., Fehm, H. L., \& Born, J. (2002). Food deprivation fails to affect preoccupation with thoughts of food in anorectic patients. The British Journal of Clinical Psychology, 41, 321-326.

Pinel, J. P., Assanand, S., \& Lehman, D. R. (2000). Hunger, eating, and ill health. The American Psychologist, 55, 1105-1116.

Placanica, J. L., Faunce, G. J., \& Soames Job, R. F. (2002). The effect of fasting on attentional biases for food and body shape/weight words in high and low Eating Disorder Inventory scorers. The International Journal of Eating Disorders, 32, 79-90.

Popper, K. R. (1972). The logic of scientific discovery. London: Hutchinson \& Co.

Porubska, K., Veit, R., Preissl, H., Fritsche, A., \& Birbaumer, N. (2006). Subjective feeling of appetite modulates brain activity: an fMRI study. Neuroimage, 32, 1273-1280. 
Posner, M. I., \& Petersen, S. E. (1990). The attention system of the human brain. Annual Review of Neuroscience, 13, 25-42.

Prentice, A. M. (1998). Manipulation of dietary fat and energy density and subsequent effects on substrate flux and food intake. The American Journal of Clinical Nutrition, $67,535 S-541 \mathrm{~S}$.

Pujol, J., Harrison, B. J., Ortiz, H., Deus, J., Soriano-Mas, C., Lopez-Sola, M., et al. (2009). Influence of the fusiform gyrus on amygdala response to emotional faces in the nonclinical range of social anxiety. Psychological Medicine, 39, 1177-1187.

Rainer, G., Augath, M., Trinath, T., \& Logothetis, N. K. (2001). Nonmonotonic noise tuning of BOLD fMRI signal to natural images in the visual cortex of the anesthetized monkey. Current Biology, 11, 846-854.

Rauch, A. V., Ohrmann, P., Bauer, J., Kugel, H., Engelien, A., Arolt, V., et al. (2007). Cognitive coping style modulates neural responses to emotional faces in healthy humans: a 3-T FMRI study. Cerebral Cortex, 17, 2526-2535.

Rieger, E., Schotte, D. E., Touyz, S. W., Beumont, P. J., Griffiths, R., \& Russell, J. (1998). Attentional biases in eating disorders: a visual probe detection procedure. The International Journal of Eating Disorders, 23, 199-205.

Rinck, M., Reinecke, A., Ellwart, T., Heuer, K., \& Becker, E. S. (2005). Speeded detection and increased distraction in fear of spiders: evidence from eye movements. Journal of Abnormal Psychology, 114, 235-248.

Roberts, M. E., Tchanturia, K., Stahl, D., Southgate, L., \& Treasure, J. (2007). A systematic review and meta-analysis of set-shifting ability in eating disorders. Psychological Medicine, 37, 1075-1084.

Robinson, T. E., \& Berridge, K. C. (1993). The neural basis of drug craving: an incentivesensitization theory of addiction. Brain Research. Brain Research Reviews, 18, $247-$ 291.

Robinson, T. E., \& Berridge, K. C. (2000). The psychology and neurobiology of addiction: an incentive-sensitization view. Addiction, 95, 91-117.

Robinson, T. E., \& Berridge, K. C. (2001). Incentive-sensitization and addiction. Addiction, 96, 103-114.

Robinson, T. E., \& Berridge, K. C. (2003). Addiction. Annual Review Psychology, 54, $25-$ 53.

Roefs, A., Herman, C. P., Macleod, C. M., Smulders, F. T., \& Jansen, A. (2005). At first sight: how do restrained eaters evaluate high-fat palatable foods? Appetite, 44, 103114. 
Roefs, A., Quaedackers, L., Werrij, M. Q., Wolters, G., Havermans, R., Nederkoorn, C., et al. (2006). The environment influences whether high-fat foods are associated with palatable or with unhealthy. Behavior Research and Therapy, 44, 715-736.

Rolls, B. J. (2000). The role of energy density in the overconsumption of fat. The Journal of Nutrition, 130, 268S-271S.

Rolls, B. J., Laster, L. J., \& Summerfelt, A. (1989). Hunger and food intake following consumption of low-calorie foods. Appetite, 13, 115-127.

Rolls, E. T. (2007). Sensory processing in the brain related to the control of food intake. The Proceedings of the Nutrition Society, 66, 96-112.

Rolls, E. T., Murzi, E., Yaxley, S., Thorpe, S. J., \& Simpson, S. J. (1986). Sensory-specific satiety: food-specific reduction in responsiveness of ventral forebrain neurons after feeding in the monkey. Brain Research, 368, 79-86.

Rosse, R. B., Johri, S., Kendrick, K., Hess, A. L., Alim, T. N., Miller, M., et al. (1997). Preattentive and attentive eye movements during visual scanning of a cocaine cue: correlation with intensity of cocaine cravings. The Journal of Neuropsychiatry and Clinical Neurosciences, 9, 91-93.

Rosse, R. B., Miller, M. W., Hess, A. L., Alim, T. N., \& Deutsch, S. I. (1993). Measures of visual scanning as a predictor of cocaine cravings and urges. Biological Psychiatry, 33, 554-556.

Rothemund, Y., Preuschhof, C., Bohner, G., Bauknecht, H. C., Klingebiel, R., Flor, H., et al. (2007). Differential activation of the dorsal striatum by high-calorie visual food stimuli in obese individuals. Neuroimage, 37, 410-421.

Russell, T. A., Schmidt, U., Doherty, L., Young, V., \& Tchanturia, K. (2009). Aspects of social cognition in anorexia nervosa: affective and cognitive theory of mind. Psychiatry Research, 168, 181-185.

Salamone, J. D., Correa, M., Mingote, S., \& Weber, S. M. (2003). Nucleus accumbens dopamine and the regulation of effort in food-seeking behavior: implications for studies of natural motivation, psychiatry, and drug abuse. The Journal of Pharmacology and Experimental Therapeutics, 305, 1-8.

Salamone, J. D., Correa, M., Mingote, S. M., \& Weber, S. M. (2005). Beyond the reward hypothesis: alternative functions of nucleus accumbens dopamine. Current Opinion in Pharmacology, 5, 34-41.

Santel, S., Baving, L., Krauel, K., Munte, T. F., \& Rotte, M. (2006). Hunger and satiety in anorexia nervosa: $\mathrm{fMRI}$ during cognitive processing of food pictures. Brain Research, $1114,138-148$.

Saper, C. B., Chou, T. C., \& Elmquist, J. K. (2002). The need to feed: homeostatic and hedonic control of eating. Neuron, 36, 199-211. 
Saxe, R., Brett, M., \& Kanwisher, N. (2006). Divide and conquer: a defense of functional localizers. Neuroimage, 30, 1088-1096.

Schmidt, U., \& Treasure, J. (2006). Anorexia nervosa: valued and visible. A cognitiveinterpersonal maintenance model and its implications for research and practice. The British Journal of Clinical Psychology, 45, 343-366.

Schultz, W. (2006). Behavioral theories and the neurophysiology of reward. Annual Review of Psychology, 57, 87-115.

Schwarz, N. (1999). How the questions shape the answers. American Psychologist, 54, 93105.

Schweinhardt, P., Seminowicz, D. A., Jaeger, E., Duncan, G. H., \& Bushnell, M. C. (2009). The anatomy of the mesolimbic reward system: a link between personality and the placebo analgesic response. The Journal of Neuroscience, 29, 4882-4887.

Sebastian, S. B., Williamson, D. A., \& Blouin, D. C. (1996). Memory bias for fatness stimuli in the eating disorders. Cognitive Therapy and Research, 20, 275-286.

Shafran, R., Lee, M., Cooper, Z., Palmer, R. L., \& Fairburn, C. G. (2007). Attentional bias in eating disorders. The International Journal of Eating Disorders, 40, 369-380.

Shin, A. C., Zheng, H., \& Berthoud, H. R. (2009). An expanded view of energy homeostasis: neural integration of metabolic, cognitive, and emotional drives to eat. Physiology \& Behavior, 97, 572-580.

Siep, N., Roefs, A., Roebroeck, A., Havermans, R., Bonte, M. L., \& Jansen, A. (2009). Hunger is the best spice: an fMRI study of the effects of attention, hunger and calorie content on food reward processing in the amygdala and orbitofrontal cortex. Behavioural Brain Research, 198, 149-158.

Siep, N., Roefs, A., Roebroeck, A., Havermans, R., Bonte, M. L., \& Jansen, A. (2010a). Fighting food temptations: the modulating effects of cognitive reappraisal, suppression and up-regulation on mesocorticolimbic activity related to appetitive motivation. Manuscript submitted for publication.

Siep, N., roefs, A., Roebroeck, A., Havermans, R., Bonte, M. L., \& Jansen, A. (2010b). When not eating becomes easy: decreased food reward processing versus increased inhibitory self-control in currently-ill Anorexia Nervosa patients, an fMRI study. Manuscript submitted for publication.

Simmons, W. K., Martin, A., \& Barsalou, L. W. (2005). Pictures of appetizing foods activate gustatory cortices for taste and reward. Cerebral Cortex, 15, 1602-1608.

Small, D. M., Veldhuizen, M. G., Felsted, J., Mak, Y. E., \& McGlone, F. (2008). Separable substrates for anticipatory and consummatory food chemosensation. Neuron, 57, 786-797. 
Small, D. M., Zatorre, R. J., Dagher, A., Evans, A. C., \& Jones-Gotman, M. (2001). Changes in brain activity related to eating chocolate: from pleasure to aversion. Brain, 124, 1720-1733.

Small, D. M., Zatorre, R. J., \& Jones-Gotman, M. (2001). Changes in taste intensity perception following anterior temporal lobe removal in humans. Chemical Senses, 26, 425-432.

Smeets, E., Jansen, A., Lindelauf, T., \& Roefs, A. (2010). Selective attention for unattractive body parts causes body dissatisfaction. Paper submitted for publication.

Smeets, E., Roefs, A., van Furth, E., \& Jansen, A. (2008). Attentional bias for body and food in eating disorders: increased distraction, speeded detection, or both? Behaviour Research and Therapy, 46, 229-238.

Smeets, E., Tiggemann, M., Kemps, E., Mills, J. S., Hollitt, S., Roefs, A., et al. (2009). Body checking induces an attentional bias for body-related cues. The International Journal of Eating Disorders. In press.

Spiegler, B. J., \& Mishkin, M. (1981). Evidence for the sequential participation of inferior temporal cortex and amygdala in the acquisition of stimulus-reward associations. Behavioural Brain Research, 3, 303-317.

Spinella, M. (2007). Normative data and a short form of the Barratt Impulsiveness Scale. The International Journal of Neuroscience, 117, 359-368.

Spyraki, C., Fibiger, H. C., \& Phillips, A. G. (1982). Attenuation by haloperidol of place preference conditioning using food reinforcement. Psychopharmacology, 77, 379382.

Stahre, L., \& Hallstrom, T. (2005). A short-term cognitive group treatment program gives substantial weight reduction up to 18 months from the end of treatment. A randomized controlled trial. Eating and Weight Disorders, 10, 51-58.

Steinhausen, H. C., \& Weber, S. (2009). The outcome of bulimia nervosa: findings from one-quarter century of research. American Journal of Psychiatry, 166, 1331-1341.

Stephens, M. (2007). Think Slim. Melbourne: Allen \& Unwin.

Stice, E., Spoor, S., Bohon, C., \& Small, D. M. (2008). Relation between obesity and blunted striatal response to food is moderated by TaqIA A1 allele. Science, 322, 449452.

Stice, E., Spoor, S., Ng, J., \& Zald, D. H. (2009). Relation of obesity to consummatory and anticipatory food reward. Physiology \& Behavior, 97, 551-560.

Stoeckel, L. E., Weller, R. E., Cook, E. W., 3rd, Twieg, D. B., Knowlton, R. C., \& Cox, J. E. (2008). Widespread reward-system activation in obese women in response to pictures of high-calorie foods. Neuroimage, 41, 636-647. 
Sturm, R. (2002). The effects of obesity, smoking, and drinking on medical problems and costs. Health Affairs, 21, 245-253.

Suslow, T., Ohrmann, P., Lalee-Mentzel, J., Donges, U. S., Arolt, V., \& Kersting, A. (2004). Incidental learning of food and emotional words in women with anorexia nervosa. Eating and Weight Disorders, 9, 290-295.

Talairach, J., \& Tournoux, P. (1988). Co-Planar Stereotaxic Atlas of the Human Brain. New York.

Tapert, S. F., Pulido, C., Paulus, M. P., Schuckit, M. A., \& Burke, C. (2004). Level of response to alcohol and brain response during visual working memory. Journal of Studies on Alcohol, 65, 692-700.

Tataranni, P. A., Gautier, J. F., Chen, K., Uecker, A., Bandy, D., Salbe, A. D., et al. (1999). Neuroanatomical correlates of hunger and satiation in humans using positron emission tomography. Proceedings of the National Academy of Sciences of the United States of America, 96, 4569-4574.

Tchanturia, K., Anderluh, M. B., Morris, R. G., Rabe-Hesketh, S., Collier, D. A., Sanchez, P., et al. (2004). Cognitive flexibility in anorexia nervosa and bulimia nervosa. Journal of the International Neuropsychological Society, 10, 513-520.

Tchanturia, K., Davies, H., \& Campbell, I. C. (2007). Cognitive remediation therapy for patients with anorexia nervosa: preliminary findings. Annals of General Psychiatry, 6, 14.

Tchanturia, K., Morris, R. G., Anderluh, M. B., Collier, D. A., Nikolaou, V., \& Treasure, J. (2004). Set shifting in anorexia nervosa: an examination before and after weight gain, in full recovery and relationship to childhood and adult OCPD traits. Journal of Psychiatric Research, 38, 545-552.

Tiggemann, M., \& Kemps, E. (2005). The phenomenology of food cravings: the role of mental imagery. Appetite, 45, 305-313.

Toates, F. (1986). Motivational systems. Cambridge (MA): Cambridge University Press.

Toates, F. M. (1981). The control of ingestive behaviour by internal and external stimuli A theoretical review. Appetite, 2, 35-50.

Treasure, J. L. (2007). Getting beneath the phenotype of anorexia nervosa: the search for viable endophenotypes and genotypes. Canadian Journal of Psychiatry, 52, 212-219.

Treisman, A. M., \& Gelade, G. (1980). A feature-integration theory of attention. Cognitive Psychology, 12, 97-136.

Tsai, S. J. (2005). Repetitive transcranial magnetic stimulation: a possible novel therapeutic approach to eating disorders. Medical Hypotheses, 65, 1176-1178. 
Uher, R., Murphy, T., Brammer, M. J., Dalgleish, T., Phillips, M. L., Ng, V. W., et al. (2004). Medial prefrontal cortex activity associated with symptom provocation in eating disorders. American Journal of Psychiatry, 161, 1238-1246.

Valenstein, E. S., Cox, V. C., \& Kakolewski, J. W. (1970). Reexamination of the role of the hypothalamus in motivation. Psycholigical Review, 77, 16-31.

Vitousek, K., \& Hollon, S. (1990). The investigation of schematic content and processing in eating disorders. Cognitive Therapy and Research. , 14, 191-214.

Volkow, N. D., Wang, G. J., Telang, F., Fowler, J. S., Goldstein, R. Z., Alia-Klein, N., et al. (2008). Inverse Association Between BMI and Prefrontal Metabolic Activity in Healthy Adults. Obesity, 17, 60-65.

Volkow, N. D., Wang, G. J., Telang, F., Fowler, J. S., Thanos, P. K., Logan, J., et al. (2008). Low dopamine striatal D2 receptors are associated with prefrontal metabolism in obese subjects: possible contributing factors. Neuroimage, 42, 1537-1543.

Volkow, N. D., \& Wise, R. A. (2005). How can drug addiction help us understand obesity? Nature Neuroscience, 8, 555-560.

Vuilleumier, P., Richardson, M. P., Armony, J. L., Driver, J., \& Dolan, R. J. (2004). Distant influences of amygdala lesion on visual cortical activation during emotional face processing. Nature Neuroscience, 7, 1271-1278.

Wager, T. D., Davidson, M. L., Hughes, B. L., Lindquist, M. A., \& Ochsner, K. N. (2008). Prefrontal-subcortical pathways mediating successful emotion regulation. Neuron, 59, 1037-1050.

Wagner, A., Aizenstein, H., Mazurkewicz, L., Fudge, J., Frank, G. K., Putnam, K., et al. (2008). Altered insula response to taste stimuli in individuals recovered from restricting-type anorexia nervosa. Neuropsychopharmacology, 33, 513-523.

Wagner, A., Aizenstein, H., Venkatraman, V. K., Fudge, J., May, J. C., Mazurkewicz, L., et al. (2007). Altered reward processing in women recovered from anorexia nervosa. American Journal of Psychiatry, 164, 1842-1849.

Wagner, A., Barbarich-Marsteller, N. C., Frank, G. K., Bailer, U. F., Wonderlich, S. A., Crosby, R. D., et al. (2006). Personality traits after recovery from eating disorders: do subtypes differ? The International Journal of Eating Disorders, 39, 276-284.

Walton, M. E., Groves, J., Jennings, K. A., Croxson, P. L., Sharp, T., Rushworth, M. F., et al. (2009). Comparing the role of the anterior cingulate cortex and 6hydroxydopamine nucleus accumbens lesions on operant effort-based decision making. The European Journal of Neuroscience, 29, 1678-1691.

Wang, G. J., Volkow, N. D., Logan, J., Pappas, N. R., Wong, C. T., Zhu, W., et al. (2001). Brain dopamine and obesity. Lancet, 357, 354-357. 
Wang, G. J., Volkow, N. D., Telang, F., Jayne, M., Ma, J., Rao, M., et al. (2004). Exposure to appetitive food stimuli markedly activates the human brain. Neuroimage, 21, 1790-1797.

Wang, G. J., Volkow, N. D., Telang, F., Jayne, M., Ma, Y., Pradhan, K., et al. (2009). Evidence of gender differences in the ability to inhibit brain activation elicited by food stimulation. Proceedings of the National Academy of Sciences of the United States of America, 106, 1249-1254.

Wardle, J. (2007). Eating behaviour and obesity. Obesity Review, 8, 73-75.

Weigle, D. S., Breen, P. A., Matthys, C. C., Callahan, H. S., Meeuws, K. E., Burden, V. R., et al. (2005). A high-protein diet induces sustained reductions in appetite, ad libitum caloric intake, and body weight despite compensatory changes in diurnal plasma leptin and ghrelin concentrations. The American Journal of Clinical Nutrition, 82, 4148.

Weiskopf, N., Hutton, C., Josephs, O., \& Deichmann, R. (2006). Optimal EPI parameters for reduction of susceptibility-induced BOLD sensitivity losses: a whole-brain analysis at $3 \mathrm{~T}$ and 1.5 T. Neuroimage, 33, 493-504.

Weiskopf, N., Sitaram, R., Josephs, O., Veit, R., Scharnowski, F., Goebel, R., et al. (2007). Real-time functional magnetic resonance imaging: methods and applications. Magnetic Resonance Imaging, 25, 989-1003.

Wenzlaff, R. M., \& Wegner, D. M. (2000). Thought suppression. Annual Review of Psychology, 51, 59-91.

Werrij, M. Q., Jansen, A., Mulkens, S., Elgersma, H. J., Ament, A. J., \& Hospers, H. J. (2009). Adding cognitive therapy to dietetic treatment is associated with less relapse in obesity. J of Psychosomatic Research, 67, 315-324.

Whalen, P. J., Rauch, S. L., Etcoff, N. L., Mclnerney, S. C., Lee, M. B., \& Jenike, M. A. (1998). Masked presentations of emotional facial expressions modulate amygdala activity without explicit knowledge. The Journal of Neuroscience, 18, 411-418.

WHO. (2002). Reducing risks, promoting healthy life. Retrieved August 14, 2008, from http://www.who.int/whr/2002/en/.

Williams, J. M., Mathews, A., \& MacLeod, C. (1996). The emotional Stroop task and psychopathology. Psychological Bulletin, 120, 3-24.

Williamson, D. A., Muller, S. L., Reas, D. L., \& Thaw, J. M. (1999). Cognitive bias in eating disorders: implications for theory and treatment. Behavior Modification, 23, 556-577.

Williamson, D. A., Perrin, L., Blouin, D. C., \& Barbin, J. M. (2000). Cognitive bias in eating disorders: interpretation of ambiguous body-related information. Eating and Weight Disorders, 5, 143-151. 
Williamson, D. A., White, M. A., York-Crowe, E., \& Stewart, T. M. (2004). Cognitivebehavioral theories of eating disorders. Behavior Modification, 28, 711-738.

Wilson, G. T., Grilo, C. M., \& Vitousek, K. M. (2007). Psychological treatment of eating disorders. The American Psychologist, 62, 199-216.

Wilson, G. T., \& Shafran, R. (2005). Eating disorders guidelines from NICE. Lancet, 365, 79-81.

Wise, R. A. (1978). Catecholamine theories of reward: a critical review. Brain Research, $152,215-247$.

Wise, R. A. (2004). Dopamine, learning and motivation. Nature Reviews Neuroscience, 5, 483-494.

Wise, R. A., \& Bozarth, M. A. (1987). A psychomotor stimulant theory of addiction. Psychological Review, 94, 469-492.

Wolfe, E. L., Barger, A. C., \& Benison, S. (2000). Walter B. Cannon: Science And Society Boston: Boston Medical Library

Yeomans, M. R., Lee, M. D., Gray, R. W., \& French, S. J. (2001). Effects of test-meal palatability on compensatory eating following disguised fat and carbohydrate preloads. International Journal of Obesity and Related Metabolic Disorders, 25, 12151224.

Zastrow, A., Kaiser, S., Stippich, C., Walther, S., Herzog, W., Tchanturia, K., et al. (2009). Neural correlates of impaired cognitive-behavioral flexibility in anorexia nervosa. American Journal of Psychiatry, 166, 608-616.

Zelissen, P. M., Stenlof, K., Lean, M. E., Fogteloo, J., Keulen, E. T., Wilding, J., et al. (2005). Effect of three treatment schedules of recombinant methionyl human leptin on body weight in obese adults: a randomized, placebo-controlled trial. Diabetes, Obesity \& Metabolism, 7, 755-761.

Zhang, Y., Proenca, R., Maffei, M., Barone, M., Leopold, L., \& Friedman, J. M. (1994). Positional cloning of the mouse obese gene and its human homologue. Nature, 372, 425-432. 
Dankwoord 
Het lijkt nog als de dag van gisteren dat ik met mijn promotieonderzoek begon. Inmiddels zijn er, bijna ongemerkt, alweer 4 jaren voorbij. Mooie jaren, waarin ik veel heb geleerd, met "ups en downs" (zoals het onderzoek betaamt) en met enorm veel steun van collega's, vrienden en familie zonder wie dit allemaal niet mogelijk was geweest. Allemaal bedankt! Een aantal van hen wil ik speciaal noemen.

Mijn promotor, professor Jansen. Beste Anita, hartelijk dank voor de mogelijkheid tot het doen van mijn promotieonderzoek bij Clinical Psychological Science, voor je vertrouwen, kritische blik en motiverende gesprekken.

Mijn eerste copromotor en directe begeleider, Anne. Ik ben mij ervan bewust dat mijn enorme eigenwijsheid het voor jou niet altijd gemakkelijk maakte om mijn begeleider te zijn. Ik ben je dan ook heel erg dankbaar voor het vertrouwen dat je mij hebt gegeven. Jouw nauwkeurigheid, enorme vakkennis, eindeloze inzet en onvermoeibare voorkomen waren een bron van inspiratie.

Mijn tweede copromotor, Alard. Dankjewel voor je waardevolle hulp in het bijzonder met BrainVoyager. Ik hoop dat ik ook in de toekomst nog af en toe van jouw expertise gebruik mag maken.

Remco en Milene, ontzettend bedankt voor al jullie onmisbare inzichten en creativiteit bij het ontwerpen van de studies en schrijven van de manuscripten.

The panel of judges, professor Arntz, professor Adan, doctor Formisano, professor Goebel and professor Stice. Thank you very much for your willingness to review my thesis and for your participation in the defence.

Diegenen die ik heel hartelijk wil bedanken voor hun directe betrokkenheid bij mijn project, zijn alle proefpersonen die hebben meegedaan aan mijn onderzoeken en alle contactpersonen die hebben geholpen bij het vinden van proefpersonen. Zonder hen zou dit proefschrift er niet zijn.

Naast promotoren en professoren zijn secretaresses ook absoluut een noodzakelijke voorwaarde voor een goed promotietraject: Jessie en Truus, bedankt! 
De mannen van de technische dienst, met name Sven, Ron, Jacco en Erik, heel erg bedankt voor jullie onmisbare hulp bij het programmeren van de scripten en de snelle ondersteuning wanneer mijn "hardware wereld" weer eens dreigde in te storten.

Dear Armin, thank you so much for the numerous occasions that you helped me with BrainVoyager. You have been invaluable in the successful completion of this thesis.

Beste eetgroepers, dankjewel voor jullie geduld en luisterend oor wanneer ik weer eens kwam aanzetten met plaatjes van 'oplichtende hersenen' en betogen over dopamine. Jullie hebben mij kritisch leren nadenken over de relevantie en implicaties van het neuroimaging onderzoek.

Plezier hebben in je werk is gedeeltelijk afhankelijk van je werkomgeving. Een goede sfeer is essentieel, zeker als je met een aantal mensen op dezelfde kamer zit. Wat dat betreft heb ik het altijd enorm getroffen met mijn kamergenoten. Lieve Ramona, dankjewel voor je steun, je goede zorgen en plezante gesprekken (vooral die met betrekking tot de toekomst;)). Lieve Astrid, mede fMRI-nerd, wat heb ik om jouw vreemde capriolen moeten lachen. Maar eerlijkheid gebied te zeggen: dankzij jouw geniale ideeën (mini oerwout, iso-matje, power nap, ventilator, kruidenthee, etc.) ging het productieniveau in onze kamer significant omhoog.

Mijn twee paranimfen, Moniek en Christine. Ik ben ontzettend blij dat jullie mij op deze bijzondere dag willen bijstaan. Moniek, lief zusje, jij kent mij door en door. Jouw nuchterheid herinnert me er vaak aan wat nou echt belangrijk is in dit leven. Ik hou van jou! Lieve Christine, mijn eerste kamergenote en onmisbare partner in science. Jij bent het toonbeeld van de moderne vrouw: je bent niet alleen een geweldige wetenschapster, maar ook nog eens een sterke vrouw en fantastische moeder. Ik heb genoten van onze diepgaande gesprekken en hoop dat onze vriendschap nog heel lang mag duren.

Tot slot wil ik al mijn (schoon)familie en vrienden bedanken voor alle steun en begrip. Jullie waren er altijd om op de successen te klinken en de 
ontgoochelingen te relativeren. Math en Tilly, dankjewel voor alle opbeurende woorden, jullie oprechte interesse en de lieve krantenknipsels. Lars en Naomi, onze dromen (vooral die over dat bounty eiland met die manta ray in de achtertuin) zorgden ervoor dat ik de zon achter de tyfoon bleef zien! Titia, hopelijk gaan we elkaar, nu dit proefschrift af, is weer wat vaker zien en kunnen we eindelijk onze welverdiende bloemetjes buitenzetten. Peter, dankjewel voor je hulp bij de verbouwing, zonder jou was ik nu nog steeds aan het isoleren en was dit proefschrift nooit op tijd af geweest. Tobias, dankjewel voor je gezelschap tijdens het scannen en je geweldige humor. Saman, ik ben enorm blij dat je weer terug bent. Bij jou kan en mag ik altijd mijn ei kwijt. Tenslotte, alle 'nieuwe' vrienden die ervoor zorgden dat wij ons onmiddellijk thuis voelden in 't durp.

Lieve mamma en daddy, al 31 jaren lang staan jullie mij met raad en daad bij en kan ik altijd op jullie rekenen. Dankjewel voor alles! Jullie betekenen heel veel voor mij. Zonder jullie geduld en begrip voor mijn eeuwige waarom-fase, was dit proefschrift er nooit geweest. Daarom wil ik deze dan ook aan jullie opdragen. Paps, dankjewel voor de prachtige omslag!

Lieve Charles, de laatste vier jaren waren erg zwaar voor ons (verbouwen, verhuizen, weer verbouwen, weer verhuizen, alweer verbouwen), maar samen krijgen we alles voor elkaar en daar ben ik zo enorm trots op. Jouw enorme rust, stille kracht, optimisme, niet-humor (:P) en onvoorwaardelijke liefde geven mij het gevoel dat ik de hele wereld aan kan. Ik hou ontzettend veel van jou.

Learn as if you were to live forever,

live as if you were to die tomorrow

Mohandas Gandhi 
Curriculum Vitae 


\section{CURRICULUM VITAE}

Nicolette Siep was born on the $19^{\text {th }}$ of June 1979 in Springs, South-Africa. In 1997 she graduated from secondary school (voorgezet wetenschappelijk onderwijs, Euro College in Maastricht). In 2003 she obtained her bachelor's degree in Social Work at the Hogeschool Maastricht. In 2006 she obtained her Master's degree in Psychology (cum laude), with a specialisation in Cognitive Neuroscience. Between September 2004 and March 2008 she worked on her $\mathrm{PhD}$ project at Maastricht University, department of Clinical Psychological Science. Since April 2008 she is a post-doctoral fellow at Maastricht University, working on several clinical functional magnetic resonance imaging projects at the department of Clinical Psychological Science of Maastricht University.

\section{PUBLICATIONS}

Siep, N., Jansen, A., Havermans, R., \& Roefs, A. Cognitions and Emotions in Eating Disorders. In Kaye, W.H. \& Adan, R.A.H. (Eds.), Behavioural Neurobiology of Eating Disorders. Manuscript submitted for publication.

Siep, N., Roefs, A., Roebroeck, A., Havermans, R., Bonte, M. L., \& Jansen, A. (2009). Hunger is the best spice: an fMRI study of the effects of attention, hunger and calorie content on food reward processing in the amygdala and orbitofrontal cortex. Behavioural Brain Research, 198(1), 149-158.

Siep, N., Roefs, A., Roebroeck, A., Havermans, R., Bonte, M. L., \& Jansen, A. (2010). When not eating becomes easy: decreased food reward processing versus increased inhibitory self-control in currently-ill Anorexia Nervosa patients, an fMRI study. Manuscript submitted for publication.

Siep, N., Roefs, A., Roebroeck, A., Havermans, R., Bonte, M. L., \& Jansen, A. (2010). Fighting food temptations: the modulating effects of cognitive reappraisal, suppression and up-regulation on mesocorticolimbic activity related to appetitive motivation. Manuscript submitted for publication.

Siep, N., Roefs, A., Havermans, R. \& Jansen, A. (2008) Als het plezier in eten verdwijnt: een hypothese over de rol van voedseldevaluatie bij Anorexia Nervosa. De Psycholoog (43), 405-408.

Siep, N. Recensie A.A. van Elburg, Psychoendocrinological aspects of anorexia nervosa. Maandblad Geestelijke Volksgezondheid, 2007.

Renvall, H.M.L., Siep, N., Jensen, O., Formisano, E., Staeren, N. Cat or girl? Neural correlates of auditory categorical perception. In preparation.

Havermans, R., Siep, N., Jansen, A. Sensory-specific satiety is impervious to the tasting of other foods with its assessment. Appetite. In press. 


\section{PRESENTATIONS}

Siep, N., Roefs, A., Roebroeck, A., Havermans, R., Bonte, M.L., \& Jansen, A. (2010). Food reward in the anorextic brain. Paper presented at the annual meeting of the British Feeding and Drinking Group. Maastricht, The Netherlands.

Siep, N., Roefs, A., Roebroeck, A., Havermans, R., Bonte, M.L., \& Jansen, A. (2009). Anorexia nervosa: hyporeactivity of the corticomesolimbic food reward circuitry. Paper presented at the Nederlandse Academie voor Eetstoornissen. Amsterdam, The Netherlands.

Siep, N., Roefs, A., Roebroeck, A., Havermans, R., Bonte, M.L., \& Jansen, A. (2009). Modulating the food reward system. Paper presented at the Dutch Endo-NeuroPsycho Meeting. Doorwerth, The Netherlands.

Siep, N., Roefs, A., Roebroeck, A., Havermans, R., Bonte, M.L., \& Jansen, A. (2009). Modulating the food reward system. Invited Keynote at the annual meeting of the British Feeding and Drinking Group. Swansea, England.

Siep, N., Roefs, A., Roebroeck, A., Havermans, R., Bonte, M.L., \& Jansen, A.. (2007). An fMRI study of food reward processing and its modulation by hunger. Poster presented at the annual meeting of the "Eating Disorders Research Society". Pittsburgh, United States of America.

Siep, N., Roefs, A., Roebroeck, A., Havermans, R., Bonte, M.L., \& Jansen, A. (2007). An fMRI study of food reward processing and its modulation by hunger. Paper Presented at the World Congress of Behavioural \& Cognitive Therapies. Barcelona, Spain.

Siep, N., Roefs, A., Roebroeck, A., Havermans, R., Bonte, M.L., \& Jansen, A. (2007). An fMRI study of food reward processing and its modulation by hunger. Poster presented at the annual meeting of the British Feeding and Drinking Group. Newcastle, England.

\section{PRESENT (INTER)NATIONAL PROJECTS}

Arntz, A., Jacob, G., Siep, N., Tüscher, O., Willenborg., B., Goebel, R. Emotional dysregulation and threat bias in borderline personality disorder: changes in brain activation and information processing during the course of psychological treatment.

Firk, C., Siep, N., Markus, R Changing feelings: How the serotonin transporter modulates emotion regulation in patients with depression: An fMRI study.

Frankort, A., Roefs, A., Siep, N., Roebroeck, A., Havermans, R. \& Jansen, A. Food reward processing in normal weight and overweight participants with and without explicit taste evaluation.

Kreusch, K., Houben, K., Goffaux, V., Siep, N., Quertemont, E. \& Wiers, R. Neural mechanisms underlying attentional bias for alcohol-related cues in young heavy drinkers and its modulation by alcohol administration. 
OTHER ACADEMIC ACTIVITIES

Media appearance on a food reward and obesity issue in a Dutch public broadcasting company program Labyrint, VPRO (April, 2010).

Reviewer for peer-reviewed journals including Appetite, The Journal of Experimental Social Psychology and The American Journal of Psychiatry.

Co-applicant of external funding application entitled Emotional dysregulation and threat bias in borderline personality disorder, open research area in Europe for social sciences; NWO (April, 2010). 